\title{
ASYMPTOTIC FORMULAE WITH REMAINDER ESTIMATES FOR EIGENVALUE BRANCHES OF THE SCHRÖDINGER OPERATOR $H-\lambda W$ IN A GAP OF $H$
}

\author{
S. Z. LEVENDORSKII
}

\begin{abstract}
The Floquet theory provides a decomposition of a periodic Schrödinger operator into a direct integral, over a torus, of operators on a basic period cell. In this paper, it is proved that the same transform establishes a unitary equivalence between a multiplier by a decaying potential and a pseudo-differential operator on the torus, with an operator-valued symbol. A formula for the symbol is given.

As applications, precise remainder estimates and two-term asymptotic formulas for spectral problems for a perturbed periodic Schrödinger operator are obtained.
\end{abstract}

0.1. There are a number of articles on the discrete spectrum in a gap of the essential spectrum of the Schrödinger operator $H=-\Delta+V$ (see $[1,3,4,7,8$, $9,12,13,17,20]$ and the bibliography in $[1,3,4])$. The majority of them $[1,3$, $4,7,8,9,21]$ deal with the existence of eigenvalue branches and with estimates or asymptotics for the number $N_{ \pm}(t ; H-E ; W)$ of branches of $H \mp \mu W$ with $0<\mu<t$ which cross the energy level $E \in \mathbb{R}-\sigma(H)$ (here $W$ is a potential decaying at infinity). In $[12,13,17]$, the principal term of the asymptotics of a series of eigenvalues accumulating to a boundary point $\mathcal{E}$ of the essential spectrum was computed, without a remainder estimate.

For motivation from solid state physics (impurities in crystals), see [1] and the bibliography there.

In this paper, we obtain the principal term of the asymptotics with remainder estimate for $N_{ \pm}(t ; H-E ; W) ; W$ is smooth and non-negative, and $V$ is periodic.

The main idea of the paper is a reduction to a pseudodifferential operator (pdo) on a torus, with operator-valued symbol. For $N_{-}(t ; H-E ; W)$, further reductions allow one to obtain bounds in terms of the counting function of the spectrum of a pdo acting in sections of a finite-dimensional fibering over a torus. These bounds give the principal terms of the asymptotics as $t \rightarrow \infty$, with remainder estimates which are as sharp as those for the corresponding pdo; if for the latter an asymptotic formula with two terms of the asymptotics is known, then the same formula is valid for $N_{-}(t ; H-E ; W)$.

In [1], only the principal term of the asymptotics of $N_{-}(t ; H-E ; W)$ was found, without a remainder estimate, and $W$ was assumed to stabilize to a positive spherically homogeneous potential; on the other hand, $V$ was not necessarily periodic.

Received by the editors May 15, 1995 and, in revised form, December 9, 1995.

1991 Mathematics Subject Classification. Primary 35P20.

The author was supported in part by ISF grant RNH 000 . 
Note that the reduction to an operator with operator-valued symbol explains why the classical Weyl formula with the ordinary symbol predicts false asymptotics for $N_{-}(t ; H ; W)$ (as was observed in [1]): for the ordinary symbol under consideration, the uncertainty principle fails since derivatives of this symbol do not admit good estimates (so that the function $h$, which plays a crucial role in calculi of pseudodifferential operators [10, Chapter 18], is not small outside some compact), whereas the operator-valued symbol enjoys more favourable properties. Similar effects are well-known in the theory of degenerate elliptic operators - see, for example, the surveys $[5,15,19]$.

The same reduction and the approximate spectral projection method for pseudodifferential operators with operator-valued symbols [16, Section 22] allows one to obtain the principal term of the asymptotics of $N_{+}(t ; H-E ; W)$ when $W$ is not a short-range potential. The principal term of the asymptotics for a short-range potential $W$, was computed in [9], [3], where it was shown that it agreed with the classical Weyl formula; if $W$ is not short-range, then the classical Weyl formula fails and analogues of strong and intermediate degeneration take place (for a discussion of the types of degenerate elliptic operators, see $[5,15,19])$.

The reduction to an operator on a torus (with a subsequent reduction to different operators) also gives asymptotic formulae with remainder estimates or even two terms of the asymptotics for a series of eigenvalues of $H+W$ accumulating to a boundary point $\mathcal{E}$ of the essential spectrum of $H+W$; the corresponding results will be published elsewhere.

In [4], the asymptotics of $N_{ \pm}(t ; H-\mathcal{E} ; W)$ was computed; this case has special subtle features.

Variational arguments allow one to deduce from the results of this paper the principal term of the asymptotics for non-smooth potentials - see e.g. [19, Section 11], where the general scheme (based on results by M.S.Birman and M.Z.Solomyak) is described.

0.2. Now we formulate conditions on $V$ and $W$.

Let $\left\{\alpha^{j}\right\}_{j=1}^{n}$ be a basis in $\mathbb{R}^{n}$, and

$$
\Gamma=\left\{x \in \mathbb{R}^{n} \mid x=\sum_{j=1}^{n} p_{j} \alpha^{j}, p_{j} \in \mathbb{Z}, j=1, \ldots, n\right\}
$$

the lattice associated with $\left\{\alpha^{j}\right\}$.

Let $V$ be a real-valued $\Gamma$-periodic function of the class $L_{\infty}\left(\mathbb{R}^{n}\right)$; the corresponding multiplier will also be denoted by $V$.

$H=-\Delta+V$ is an operator in $L_{2}\left(\mathbb{R}^{n}\right)$.

Let $W$ satisfy the following conditions:

(a) There is $\rho \in(0,1]$ such that

$$
\left|\left(D^{\alpha} W\right)(x)\right| \leq C_{\alpha} W(x)\langle x\rangle^{-\rho|\alpha|}, \quad \forall \alpha,
$$

where $\langle x\rangle=\left(1+|x|^{2}\right)^{1 / 2}, C_{\alpha}$ are independent of $x$

(b) There are positive constants $m_{-}, m, d, d_{1}$ such that

$$
d_{1}\langle x\rangle^{-m_{-}} \leq W(x) \leq d\langle x\rangle^{-m} .
$$

In the last subsections of this Introduction, we consider non-negative $W$ and $W$ having singularities. 
0.3. We need the following construction which is usually used to describe $\sigma_{\text {ess }}(H)$ $=\sigma(H) \quad$ (see e.g. [18, Sect.XIII,16], and [19]).

Let $\left\{\beta^{j}\right\}_{j=1}^{n}$ be a basis dual to $\left\{\alpha^{j}\right\}_{j=1}^{n}$ :

$$
\left(\alpha^{j}, \beta^{k}\right)=2 \pi \delta_{j k},
$$

and set

$$
\Gamma^{*}=\left\{x \in \mathbb{R}^{n} \mid x=\sum_{j=1}^{n} p_{j} \beta^{j}, p_{j} \in \mathbb{Z}, j=1, \ldots, n\right\}
$$

Set

$$
\Omega=\left\{x=\sum_{j=1}^{n} t_{j} \alpha^{j} \mid t_{j} \in[0,1)\right\}, \quad \Omega^{*}=\left\{\theta=\sum_{j=1}^{n} \theta_{j} \beta^{j} \mid \theta_{j} \in[0,1)\right\} .
$$

We use the usual flat metrics on $\mathbb{R}^{n} / \Gamma$ and $\mathbb{R}^{n^{*}} / \Gamma^{*}$; when we integrate or do local considerations we identify $\mathbb{R}^{n} / \Gamma$ (resp. $\left.\mathbb{R}^{n^{*}} / \Gamma^{*}\right)$ with $\Omega$ (resp. $\Omega^{*}$ ).

For fixed $\theta \in \Omega^{*}$, denote by $\mathcal{H}(\theta)$ an operator $-\Delta+V$ on $\Omega$ with the boundary conditions

$$
u\left(x+\alpha^{j}\right)=\exp \left\{i\left(\alpha^{j}, \theta\right)\right\} u(x), \quad \partial_{t_{j}} u\left(x+\alpha^{j}\right)=\exp \left\{i\left(\alpha^{j}, \theta\right)\right\} \partial_{t_{j}} u(x)
$$

$\left(x, x+\alpha^{j} \in \partial \Omega, j=1, \ldots, n\right)$. This is a self-adjoint semibounded (from below) operator with discrete spectrum. Let $E_{1}(\theta)<E_{2}(\theta) \leq \ldots$ be its eigenvalues counted with multiplicity.

Introduce

$$
\mathfrak{L}:=\int_{\mathbb{R}^{n^{*}} / \Gamma^{*}} \oplus L_{2}(\Omega) \frac{d \theta}{\operatorname{vol} \Omega^{*}}, \quad \mathcal{H}:=\int_{\mathbb{R}^{n^{*}} / \Gamma^{*}} \oplus \mathcal{H}(\theta) \frac{d \theta}{\operatorname{vol} \Omega^{*}} .
$$

One can identify $\mathfrak{L}$ with $L_{2}\left(\mathbb{R}^{n^{*}} / \Gamma^{*} ; L_{2}(\Omega)\right)\left(\cong L_{2}\left(\Omega^{*}, d \theta / \operatorname{vol} \Omega^{*} ; L_{2}(\Omega, d x)\right)\right.$ ), where $d \theta$ and $d x$ are the Lebesgue measures over $\Omega^{*}$ and $\Omega$ respectively, and $\mathcal{H}$ with multiplication by the operator-valued function $\mathcal{H}(\cdot)$.

Define an operator $\mathcal{U}: L_{2}\left(\mathbb{R}^{n}\right) \rightarrow \mathfrak{L}$ by

$$
(\mathcal{U} f)(x, \theta)=\sum_{\gamma \in \Gamma} \exp \{-i(\theta, \gamma)\} f(x+\gamma) .
$$

This is an isometry, and $\mathcal{U} H \mathcal{U}^{*}=\mathcal{H}$. Hence

$$
\sigma(H)=\bigcup_{j \geq 1} \bigcup_{\theta \in \mathbb{R}^{n^{*}} / \Gamma^{*}} E_{j}(\theta) .
$$

0.4. Reduction to a pdo on a torus. In the paper, the computation of the spectral asymptotics is based on the following theorem, which will be proved in Section 1.

Theorem 0.1. Let $W$ satisfy (0.1). Then

$$
\mathcal{W}:=\mathcal{U} W \mathcal{U}^{*} \in L_{\rho, 0}^{-m}\left(\mathbb{R}^{n^{*}} / \Gamma^{*} ; \text { End } L_{2}(\Omega)\right)
$$

(i.e. it is a pdo on $\mathbb{R}^{n^{*}} / \Gamma^{*}$, of Hörmander's class $L_{\rho, 0}^{-m}$, with the symbol, call it $w$, taking values in End $L_{2}(\Omega)$ ), and $w$ admits an asymptotic expansion

$$
w(\xi)=W(-\xi)+\sum_{j=1}^{n}(\cdot)_{j}\left(\partial_{j} W\right)(-\xi)+\cdots,
$$

where $(\cdot)_{j}$ is a "multiplication by $x_{j}$ " operator in $L_{2}(\Omega)$. 
If in addition $W$ satisfies (0.2), then

$$
\mathcal{W}^{-1}:=\mathcal{U} W^{-1} \mathcal{U}^{*} \in L_{\rho, 0}^{m_{-}}\left(\mathbb{R}^{n^{*}} / \Gamma^{*} ; \text { End } L_{2}(\Omega)\right),
$$

and its symbol, call it $w^{-1}$, admits an asymptotic expansion

$$
w^{-1}(\xi)=W(-\xi)^{-1}+\sum_{j=1}^{n}(\cdot)_{j}\left(\partial_{j} W^{-1}\right)(-\xi)+\cdots .
$$

For a detailed formulation and a formula for the full asymptotic expansion of $w^{ \pm 1}$, see Section 1 .

0.5 . Asymptotics of $N_{-}(t ; H-E ; W)$ : a reduction to a spectral problem for a pdo acting in sections of a finite-dimensional fibering over a torus. Let $\left(\mathcal{E}^{-}, \mathcal{E}^{+}\right)$be a gap in $\sigma(H)$, and fix $E \in\left(\mathcal{E}^{-}, \mathcal{E}^{+}\right)$. Let $\mathcal{E}^{-}>-\infty$; then there is $p \geq 1$ such that

$$
E_{1}(\theta)<E_{2}(\theta) \leq \cdots \leq E_{p}(\theta)<E<E_{p+1}(\theta) \leq \ldots, \forall \theta \in \mathbb{R}^{n^{*}} / \Gamma^{*} .
$$

Let $\mathcal{P}_{1}(\theta)$ stand for a spectral projection $P_{(-\infty, E)}(\mathcal{H}(\theta))$, and set $\mathcal{L}(\theta)=\operatorname{Ran} \mathcal{P}_{1}(\theta)$ and $\mathcal{L}=\bigcup_{\theta \in \mathbb{R}^{n^{*}} / \Gamma^{*}} \mathcal{L}(\theta)$. Due to $(0.5), \mathcal{P}_{1} \in C^{\infty}\left(\mathbb{R}^{n^{*}} / \Gamma^{*} ;\right.$ End $\left.L_{2}(\Omega)\right)$ and $\operatorname{dim} \operatorname{Ran} \mathcal{P}_{1}(\theta)=p$ for all $\theta \in \mathbb{R}^{n^{*}} / \Gamma^{*}$. Hence, $\mathcal{L}$ is a $p$-dimensional fibering over $\mathbb{R}^{n^{*}} / \Gamma^{*}$, which inherits a structure of an Hermitian fibering from $L_{2}\left(\mathbb{R}^{n^{*}} / \Gamma^{*} ; L_{2}(\Omega)\right)$. Clearly, the operators

$$
u(\cdot, \theta) \mapsto \mathcal{P}_{1}(\theta)(E-\mathcal{H}(\theta))^{s} u(\cdot, \theta), \quad s \in \mathbb{R},
$$

and

$$
u(\cdot, \theta) \mapsto \mathcal{P}_{1}(\theta)\left(\mathcal{W}^{-1} u\right)(\cdot, \theta)
$$

act in $C^{\infty}\left(\mathbb{R}^{n^{*}} / \Gamma^{*} ; \mathcal{L}\right)$; we denote them by $(E-\mathcal{H})_{1}^{s}$ and $\mathcal{W}_{1}^{-1}$, respectively.

$\mathcal{W}_{1}^{-1}$ is a pdo due to Theorem 0.1 , and $(E-\mathcal{H})_{1}^{s}$ is locally a multiplier by a matrix-valued function which is positive-definite due to $(0.5)$. Hence, $\mathcal{W}_{11}^{-1}:=$ $(E-\mathcal{H})_{1}^{1 / 2} \mathcal{W}_{1}^{-1}(E-\mathcal{H})_{1}^{1 / 2}$ is a pdo acting in $C^{\infty}\left(\mathbb{R}^{n^{*}} / \Gamma^{*} ; \mathcal{L}\right)$. To calculate its local symbols, take an open set $U \subset \mathbb{R}^{n^{*}} / \Gamma^{*}$. If $\operatorname{diam} U$ is small then there exists an orthonormal basis $\left\{u_{j}(\cdot, \theta)\right\}_{j=1}^{p}$ in $\operatorname{Ran} \mathcal{P}_{1}(\theta), \theta \in U$, with $u_{j} \in C^{\infty}\left(\bar{U} ; L_{2}(\Omega)\right)$. Thus, we can define a trivialization $\chi:\left.\mathcal{L}\right|_{U} \rightarrow U \times \mathbb{C}^{p}$ by

$$
\chi^{-1}\left(\left(f_{1}(\theta), \ldots, f_{p}(\theta)\right)\right)=\sum_{j=1}^{p} u_{j}(\cdot, \theta) f_{j}(\theta) .
$$

By using (0.4) and the composition theorem for pdo (see Theorem 5.8), one obtains an asymptotic expansion for a local symbol of $\mathcal{W}_{11}^{-1}$, call it $w_{11, U, \chi}^{-1}$ :

$$
w_{11, U, \chi}^{-1}=w_{11, U, \chi}^{-1,0}+w_{11, U, \chi}^{-1,-1}+\cdots,
$$

with each term decaying faster than the previous one as $\xi \rightarrow \infty$. For detailed version of (0.6) see Section 2; here we need formulae for the first two terms only:

$$
w_{11, U, \chi}^{-1,0}(\theta, \xi)=(E-\mathcal{H}(\theta))_{11}^{1} W(-\xi)^{-1},
$$

where

and

$$
(E-\mathcal{H}(\theta))_{11}^{r}=\left[\left\langle u_{j}(\cdot, \theta),(E-\mathcal{H}(\theta))^{r} u_{l}(\cdot, \theta)\right\rangle_{L_{2}(\Omega)}\right]_{j, l=1}^{p}
$$

$$
w_{11, U, \chi}^{-1,-1}(\theta, \xi)=\left[w_{11, U, \chi ; l j}^{-1,-1}(\theta, \xi)\right]_{l, j=1}^{p}
$$




$$
\begin{aligned}
& w_{11, U, \chi ; l j}^{-1,-1}(\theta, \xi)=\sum_{r=1}^{n}\left(i D_{r} W^{-1}\right)(-\xi)\left\langle(E-\mathcal{H}(\theta))_{1}^{1 / 2} u_{l}(\cdot, \theta),\right. \\
&\left.\left(-D_{\theta_{r}}+(\cdot)_{r}\right)\left((E-\mathcal{H}(\theta))_{1}^{1 / 2} u_{j}(\cdot, \theta)\right)\right\rangle_{L_{2}(\Omega)},
\end{aligned}
$$

where $D_{j}=-i \partial_{j}$.

It follows from $(0.6)$ and $(0.2)$ that $\mathcal{W}_{11}^{-1}$ is a symmetric hypoelliptic pdo of the Hörmander class $H_{\rho, 0}^{m_{-}, m}\left(\mathbb{R}^{n^{*}} / \Gamma^{*} ; \mathcal{L}\right)$, with symbol positive definite for large $|\xi|$. Hence, the closure of $\mathcal{W}_{11}^{-1}$, call it $\overline{\mathcal{W}_{11}^{-1}}$, is a semibounded (from below) self-adjoint operator with discrete spectrum (see Theorem 5.12).

For $C \in \mathbb{R}$, set $W_{C}^{-1}(x)=W(x)^{-1}-C W(x)^{-1}\langle x\rangle^{-2 \rho}$, and construct $\mathcal{W}_{11, C}^{-1}$ in the way $\mathcal{W}_{11}^{-1}$ was constructed (but starting from $W_{C}^{-1}$ instead of $W^{-1}$ ). Theorem 0.1 gives for the symbol of $\mathcal{W}_{11, C}^{-1}$ an asymptotic expansion (0.6) with the same first terms (only the tail differs from the one for $\mathcal{W}_{11}^{-1}$ ); hence the above consideration shows that $\overline{\mathcal{W}_{11, C}^{-1}}$, the closure of $\mathcal{W}_{11, C}^{-1}$, is semibounded from below with discrete spectrum.

Denote by $N(t ; A)$ the counting function of the spectrum of a semibounded (from below) operator $A$.

Theorem 0.2. Let (0.1), (0.2) and (0.5) hold.

Then there exists $C>0$ such that

$$
N\left(t ; \overline{\mathcal{W}_{11}^{-1}}\right) \leq N_{-}(t ; H-E ; W) \leq N\left(t ; \overline{\mathcal{W}_{11, C}^{-1}}\right)
$$

0.6. Asymptotics of $N_{-}(t ; H-E ; W)$ : "hypoelliptic case". The first two terms of the asymptotic expansion (0.6) for the symbol of $\mathcal{W}_{11, C}^{-1}$ are independent of $C$; hence results for hypoelliptic pdo (see Theorem 6.4) give the same asymptotic formulae for the left- and right-most parts of (0.7) ("the same" means that only a constant in the $O$-term (or $o$-term) depends on $C$ ). Therefore, the same formulae hold for $N_{-}(t ; H-E ; W)$.

In a general "hypoelliptic case" (0.1), (0.2), we have the following theorem.

Theorem 0.3. Let (0.1), (0.2) and (0.5) hold, and let

$$
\begin{aligned}
c_{-}(t, & H-E, W)) \\
& =(2 \pi)^{-n} \sum_{j=1}^{p} \operatorname{meas}\left\{(\theta, \xi) \in \Omega^{*} \times \mathbb{R}^{n} \mid 1+t W(-\xi)\left(E_{j}(\theta)-E\right)^{-1}<0\right\}
\end{aligned}
$$

satisfy a Tauberian condition: for any $\epsilon>0$, there is $\omega>0$ such that

$$
\begin{aligned}
& c_{-}\left(t \pm t^{1-\epsilon}, H-E, W\right)-c_{-}(t, H-E, W) \\
& \quad=O\left(c_{-}(t, H-E, W) t^{-\omega}\right), \quad \text { as } t \rightarrow+\infty .
\end{aligned}
$$

Then there exists $\omega>0$ such that as $t \rightarrow+\infty$,

$$
N_{-}(t ; H-E ; W)=c_{-}(t, H-E, W)\left(1+O\left(t^{-\omega}\right)\right) .
$$

For the detailed proof, see Section 2. 
0.7. Asymptotics of $N_{-}(t ; H-E ; W)$ : "elliptic case". Let $W$ be "elliptic", i.e. let it admit a decomposition

$$
W(x)=W_{-m}(x)+O\left(|x|^{-m-1}\right), \quad \text { as }|x| \rightarrow+\infty,
$$

where $W_{-m} \in C^{\infty}\left(\mathbb{R}^{n} \backslash 0 ; \mathbb{R}_{+}\right)$is positively homogeneous of degree $-m<0$. Then (0.6) gives

$$
w_{11, U, \chi}^{-1}(\theta, \xi)=(E-\mathcal{H}(\theta))_{11}^{1} W_{-m}(-\xi)^{-1}+O\left(|\xi|^{m-1}\right), \quad \text { as }|\xi| \rightarrow+\infty,
$$

and the well-known results for elliptic pdo $[11,19]$ give the following theorem.

Theorem 0.4. Let $(0.5)$ hold, let $(0<) W \in L_{\infty}$ satisfy $(0.10)$ with $m>0$, and let $W^{-1} \in L_{\infty, l o c}\left(\mathbb{R}^{n}\right)$.

Then

$$
N_{-}(t ; H-E ; W)=c_{-}\left(H-E ; W_{-m}\right) t^{n / m}+O\left(t^{(n-1) / m}\right),
$$

where

$$
\begin{aligned}
c_{-}(H & \left.-E ; W_{-m}\right) \\
& =(2 \pi)^{-n} n^{-1} \sum_{j=1}^{p} \int_{S_{n-1}}\left(W(x)_{-m}\right)^{n / m} d S(x) \int_{\mathbb{R}^{n^{*}} / \Gamma^{*}}\left(E-E_{j}(\theta)\right)^{-n / m} d \theta .
\end{aligned}
$$

For details, see Section 2.

Now, let

$$
W(x)=W_{-m}(x)+W_{-m-1}(x)+O\left(|x|^{-m-1-\epsilon}\right), \quad \text { as }|x| \rightarrow+\infty,
$$

where $W_{j} \in C^{\infty}\left(\mathbb{R}^{n} \backslash 0\right)$ are positively homogeneous of degree $j(j=-m,-m-1)$, $\epsilon>0$, and $W_{-m}$ is positive. Then (0.6) implies that $\mathcal{W}_{11, C}^{-1}$ is (modulo terms of order less than $m-1$ ) a positive definite classical elliptic pdo of order $m$, with the principal symbol

$$
w_{11, U, \chi ; m}^{-1}(\theta, \xi)=(E-\mathcal{H}(\theta))_{11}^{1} W_{-m}(-\xi)^{-1},
$$

and the next term of the asymptotic expansion of the symbol defined locally by

$$
w_{11, U, \chi ; m-1}^{-1}(\theta, \xi)=-(E-\mathcal{H}(\theta))_{11}^{1}\left(W_{-m}^{-2} W_{-m-1}\right)(-\xi)+w_{11, U, \chi}^{\prime}(\theta, \xi),
$$

where

$$
\begin{gathered}
w_{11, U, \chi}^{\prime}(\theta, \xi)=\left[w_{11, U, \chi ; l j}^{\prime}(\theta, \xi)\right]_{l, j=1}^{p}, \\
w_{11, U, \chi ; l j}^{\prime}(\theta, \xi)=\sum_{r=1}^{n}\left(i D_{r} W_{-m}^{-1}\right)(-\xi) \\
\times\left\langle(E-\mathcal{H}(\theta))_{1}^{1 / 2} u_{l}(\cdot, \theta),\left(-D_{\theta_{r}}+(\cdot)_{r}\right)\left((E-\mathcal{H}(\theta))_{1}^{1 / 2} u_{j}(\cdot, \theta)\right)\right\rangle_{L_{2}(\Omega)} .
\end{gathered}
$$

For details, see Section 2.

If one knows the first two terms of the asymptotic expansion of the symbol of a positive definite pdo $\mathcal{W}_{11, C}^{-1}$ acting in sections of a finite-dimensional fibering over a compact manifold without boundary, one can compute both the principal and subprincipal symbols of a power $\mathcal{W}_{1}:=\left(\mathcal{W}_{11}^{-1}\right)^{1 / m}$ :

$$
w_{1, U, \chi ; p}=\left(w_{11, U, \chi ; m}^{-1}\right)^{1 / m},
$$




$$
w_{1, U, \chi, s}=w_{1, U, \chi ; 0}+\frac{i}{2} \sum_{|\alpha|=1} \partial_{\theta}^{\alpha} \partial_{\xi}^{\alpha} w_{1, U, \chi ; p}
$$

where $w_{1, U, \chi ; 0}$ is the term of order zero in the asymptotic expansion of the symbol of $\mathcal{W}_{1}$; for the formula for $w_{1, U, \chi ; 0}$, see [20]. Formulae (0.13)-(0.16) taken together provide complete information about the principal and subprincipal symbols of $\mathcal{W}_{1}$. In particular, the eigenvalues of the principal symbol of $\mathcal{W}_{1}$ are given by

$$
\mu_{j}(\theta, \xi)=\left(E-E_{j}(\theta)\right)^{1 / m}\left(W_{-m}^{-1}(-\xi)\right)^{1 / m}, \quad j=1, \ldots, p .
$$

Under certain non-periodicity condition on bicharacteristics of $\mu_{j}$, there exist general two-term asymptotic formulas for the counting function of the spectrum of elliptic operators [11] (see also [19]), but in the case of non-constant multiplicities of $\mu_{j}$, the result is only announced in [11].

By applying results of [11] to (0.7), we obtain the following theorem (modulo reservations in the case of non-constant multiplicities of $\mu_{j}$ ).

Theorem 0.5. Let $(0.5)$ hold, let $(0<) W \in L_{\infty}\left(\mathbb{R}^{n}\right)$ satisfy $(0.12)$ with $m>0$, let $W^{-1} \in L_{\infty, l o c}\left(\mathbb{R}^{n}\right)$, and let the bicharacteristics of $\mu_{j}, j=1, \ldots, p$, satisfy a non-periodicity condition of $[11,19]$.

Then

$$
\begin{gathered}
N_{-}(t ; H-E ; W)=c_{-}\left(H-E ; W_{-m}\right) t^{n / m} \\
+c_{1}\left(H-E ; W_{-m} ; W_{-m-1}\right) t^{(n-1) / m}+o\left(t^{(n-1) / m}\right) .
\end{gathered}
$$

For a (rather complicated) formula for $c_{1}\left(H-E ; W_{-m} ; W_{-m-1}\right)$ in terms of $w_{1, p}$ and $w_{1, s}$, see [11].

0.8. Asymptotics of $N_{+}(t ; H-E ; W)$. For the case $W$ a short-range potential, the principal term of asymptotics was calculated in $[9,3] ; V$ was not necessarily periodic. In [9], $W(x) \leq C\langle x\rangle^{-m}, m>2$, and in [3], $W \in L_{n / 2}\left(\mathbb{R}^{n}\right), n \geq 3$. The result of $[9,3]$ reads

$$
N_{+}(t ; H-E ; W)=t^{n / 2}(2 \pi)^{-n}\left|v_{n}\right| \int_{\mathbb{R}^{n}} W^{n / 2}(x) d x+o\left(t^{n / 2}\right), \text { as } t \rightarrow+\infty,
$$

where $\left|v_{n}\right|$ is the volume of the unit ball in $\mathbb{R}^{n}$.

If $W$ satisfies (0.1), (0.2) and there exist $C, c>0$ and a cone $K$ such that

$$
W(x) \geq c\langle x\rangle^{-m}, \quad \forall x \in K,|x| \geq C,
$$

this integral converges if and only if $m>2$. We calculate the principal term of the asymptotics, with a remainder estimate, in cases when $m \leq 2$.

Theorem 0.6. Let $W$ obey (0.1) and (0.2) with $m=2$, and let $W$ admit a representation

$$
W(x)=W_{-2}(x)+W^{\prime}(x),
$$

where $W_{-2}$ is positively homogeneous of degree -2 , positive on some set, and

$$
\left|W^{\prime}(x)\right| \leq C|x|^{-2-\epsilon},
$$

for some $\epsilon>0$.

Then

$$
N_{+}(t ; H-E ; W)=c\left(W_{-2}\right) t^{n / 2}(\ln t+O(1)), \quad \text { as } t \rightarrow+\infty,
$$


where

$$
c\left(W_{-2}\right)=(2 \pi)^{-n}\left|v_{n}\right| 2^{-1} \int_{S_{n-1}} W_{-2}(x)^{n / 2} d x .
$$

Theorem 0.7. Let $W$ obey (0.1) and (0.2) with $m \in(0,2)$ and admit a representation

$$
W(x)=W_{-m}(x)+W^{\prime}(x),
$$

where $W_{-m}$ is positively homogeneous of degree $-m$, positive on some set, and

$$
\left|W^{\prime}(x)\right| \leq C|x|^{-m-\epsilon},
$$

for some $\epsilon>0$.

Then there exists $\omega>0$ such that

$$
N_{+}(t ; H-E ; W)=c_{+}\left(H-E, W_{-m}\right) t^{n / m}\left(1+O\left(t^{-\omega}\right)\right), \text { as } t \rightarrow+\infty,
$$

where

$$
\begin{aligned}
c_{+}(H & \left.-E, W_{-m}\right) \\
& =(2 \pi)^{-n} n^{-1} \int_{S_{n-1}} W_{-m}(x)^{n / m} d x \int_{\mathbb{R}^{n^{*}} / \Gamma^{*}} \sum_{j=p+1}^{+\infty}\left(E_{j}(\theta)-E\right)^{-n / m} d \theta,
\end{aligned}
$$

and $p$ is the same as in (0.5).

Remark 0.1. The series in (0.27) converges if and only if $m<2$, and the integral in (0.18) converges if and only if $m>2$, so $m=2$ is an "intermediate case" between these two essentially different cases. Similar three cases arise in the theory of degenerate elliptic operators (see $[5,15,19]$ ).

Remark 0.2. Theorem 0.6 is valid for any $V \in L_{\infty}\left(\mathbb{R}^{n}\right)$.

Theorem 0.6 will be proved in Subsection 3.1, using a rather elementary method. The "strong degeneration case" $m<2$, when the principal term of asymptotics is expressed via an operator-valued symbol, requires a heavier machinery. In Subsection 3.2, we shall use a modification of the proof of the general theorem of the approximate spectral projection method [16, Section 22], for pdo with operatorvalued symbols. In fact, we shall prove the following result which is a bit more general than Theorem 0.7, and then derive Theorem 0.7 from it.

Theorem 0.8. Let $W$ obey (0.1), (0.2) and (0.19) with $m \in(0,2)$, and let

$$
\begin{gathered}
c_{+}(t ; H-E ; W) \\
=(2 \pi)^{-n} \sum_{j=p+1}^{+\infty} \operatorname{meas}\left\{(x, \theta) \in \mathbb{R}^{n} \times \mathbb{R}^{n^{*}} / \Gamma^{*} \mid 1-t W(x)\left(E_{j}(\theta)-E\right)^{-1}<0\right\}
\end{gathered}
$$

where $p$ is the same as in (0.5), satisfy a Tauberian condition (0.8).

Then there exists $\omega>0$ such that

$$
N_{+}(t ; H-E ; W)=c_{+}(t ; H-E ; W)\left(1+O\left(t^{-\omega}\right)\right) \text {, as } t \rightarrow+\infty \text {. }
$$


0.9. The case of non-negative $W$.

Theorem 0.9. Let $W \geq 0$ satisfy (0.19) and the condition

$$
\left|D^{\alpha} W(x)\right| \leq C_{\alpha}\langle x\rangle^{-m-|\alpha| \rho}, \quad \forall \alpha .
$$

Then:

a) if $m>0$ and (0.8) holds, then (0.9) holds;

b) if $m=2$, and (0.20) and (0.21) hold, then (0.22) holds but with $o(\ln t)$ instead of $O(1)$;

c) if $m \in(0,2)$ and $c_{+}(t ; H-E ; W)$ satisfies $(0.8)$, then $(0.29)$ holds; in particular, if (0.24) and (0.25) hold then (0.26) holds.

0.10 . Singular perturbations. For $\gamma \in \Gamma$ and $W_{1}: \mathbb{R}^{n} \rightarrow \mathbb{R}$, define a multiplier in $L_{2}(\Omega)$ :

$$
W_{1}(\cdot+\gamma): u(x) \mapsto W_{1}(x+\gamma) u(x),
$$

and set

$$
\left(\delta_{j} W_{1}\right)(\cdot+\gamma)=W_{1}\left(\cdot+\gamma+\alpha^{j}\right)-W_{1}(\cdot+\gamma)
$$

Theorem 0.10. Let $W$ satisfy (0.1), (0.2), and let $W_{1} \geq 0$ satisfy

$$
\left\|\delta_{i_{1}} \cdots \delta_{i_{l}} W_{1}(\cdot+\gamma)\right\|_{\text {End } L_{2}(\Omega)} \leq C_{p} W(\gamma)\langle\gamma\rangle^{-\epsilon-\rho l},
$$

where $\epsilon>0$ is independent of $l, i_{1}, \ldots, i_{l}$.

Then all the statements of Theorems 0.3 and $0.6-0.9$ remain valid for $N_{ \pm}\left(t ; H-E ; W+W_{1}\right)$, the right-hand sides of all the asymptotic formulae remaining expressed via $W$.

The sharp remainder estimate (0.11) holds if $\epsilon=1$, and a two-termed asymptotic formula (0.17) holds if $\epsilon>1$.

0.11. In Section 1, we study a pdo on a torus. We prove Theorems $0.2-0.5$ in Section 2, Theorems 0.6-0.8 in Section 3, and Theorems 0.9 and 0.10 in Section 4.

In Section 5 we list necessary theorems of a calculus of pdo with operator-valued symbols, and in Section 6 we state two general theorems of the approximate spectral projection method for pdo in $\mathbb{R}^{n}$ [16] and prove two analogues of one of them for pdo on a torus.

\section{ACKNOWLEDGEMENTS}

The author thanks Tony Sudbery of the University of York, and Jochen Brüning of Universität Augsburg, for their kind hospitality during the first and last stages of the work, respectively.

\section{A STUdy OF A PDO ON A TORUS}

1.1. Let $\rho \in(0,1]$, and let $W: \mathbb{R}^{n} \rightarrow \mathbb{C}$ and $f \in C^{1}\left(\mathbb{R}^{n}\right)$ satisfy the following conditions:

(a) For any $l \in \mathbb{Z}_{+}$,

$$
c_{l}(W ; f ; \rho):=\max \sup _{\gamma \in \Gamma}\left\|\delta_{j_{1}} \cdots \delta_{j_{l}} W(\cdot+\gamma)\right\|_{\operatorname{End} L_{2}(\Omega)} f(\gamma)^{-1}\langle\gamma\rangle^{\rho l}<\infty,
$$

with max taken over all $l$-tuples of integers belonging to $\{1, \ldots, n\}$.

(b) There is $C$ such that

$$
|(\nabla f)(x)| \leq C f(x)\langle x\rangle^{-\rho} .
$$


(c) There are $d_{1}>0, d, m_{1}, m$ such that

$$
d_{1}\langle x\rangle^{-m_{1}} \leq f(x) \leq d\langle x\rangle^{-m} .
$$

We write $W \in \mathcal{F}_{\rho}(f) . \mathcal{F}_{\rho}(f)$ is a Frechet space with $c_{j}(\cdot ; f ; \rho)$ as seminorms.

Let $\mathcal{U}$ be defined by $(0.3)$. We set $\mathcal{W}:=\mathcal{U} W \mathcal{U}^{*}$. It acts as follows:

$$
(\mathcal{W} u)(x, \theta)=\frac{1}{\operatorname{vol} \Omega^{*}} \sum_{\gamma \in \Gamma} W(x+\gamma) \int_{\Omega^{*}} \exp \left\{i\left(\theta^{\prime}-\theta, \gamma\right)\right\} u\left(x, \theta^{\prime}\right) d \theta^{\prime} .
$$

We shall regard $\mathcal{W}$ as an operator in $L_{2}\left(\mathbb{R}^{n^{*}} / \Gamma^{*} ; L_{2}(\Omega)\right)$. The aim of this section is to show that $\mathcal{W}$ is a pdo on $\mathbb{R}^{n^{*}} / \Gamma^{*}$, a compact manifold without boundary, with the symbol taking values in End $L_{2}(\Omega)$, the space of bounded linear operators in $L_{2}(\Omega)$. We shall use local charts

$$
\left.\kappa_{j}:\left(\mathbb{R}^{n} \supset\right) U_{j} \rightarrow U_{j}^{1}\left(\subset \mathbb{R}^{n^{*}} / \Gamma^{*}\right)\right)
$$

such that $\kappa_{s}^{-1} \circ \kappa_{r}$ is the restriction of a linear operator in $\mathbb{R}^{n}$; for $\kappa$ a local chart,

$$
\kappa^{*}: C_{0}^{\infty}\left(U^{1}\right) \rightarrow C_{0}^{\infty}(U), \quad\left(\kappa^{-1}\right)^{*}: C_{0}^{\infty}(U) \rightarrow C_{0}^{\infty}\left(U^{1}\right)
$$

stand for the corresponding maps.

We shall also need the following simple corollary of (1.2)-(1.3).

Lemma 1.1. There are $C=C(f), N=N(f)$ such that for all $x, y$,

$$
f(y) \leq C f(x)(1+|x-y|)^{N} .
$$

Proof. Clearly, there is $C_{1}>0$ such that

$$
\langle x\rangle^{\rho}\langle y\rangle^{-\rho} \leq C_{1}, \text { provided }|x-y|<\langle x\rangle^{\rho} / 2 .
$$

Fix $c<\min \left\{1 / 2,\left(C C_{1}\right)^{-1}\right\}$. Taking the supremum over a set of all $(x, y)$ with $|x-y|<c\langle x\rangle^{\rho}$ and applying the Lagrange formula and (1.2), we obtain

$$
\begin{aligned}
\sup f(x)^{-1} f(y) & \leq 1+\sup f(x)^{-1}|f(y)-f(x)| \\
& \leq 1+c\langle x\rangle^{\rho} f(x)^{-1} \sup |\nabla f(y)| \\
& \leq 1+c C\langle x\rangle^{\rho} f(x)^{-1} \sup f(y)\langle y\rangle^{-\rho} \\
& \leq 1+c C C_{1} \sup f(x)^{-1} f(y) .
\end{aligned}
$$

Hence,

$$
f(y) \leq\left(1-c C C_{1}\right)^{-1} f(x), \quad \text { if } \quad|x-y| \leq c\langle x\rangle^{\rho} .
$$

Clearly, $f^{-1}$ also satisfies (1.2). Therefore, applying the previous argument to $f^{-1}$, we find $c>0$ and $C_{2}$ such that

$$
f(y) \leq C_{2} f(x), \quad \text { if } \quad|x-y| \leq c\langle y\rangle^{\rho} .
$$

Thus, (1.4) holds if $|x-y| \leq c \max \left\{\langle x\rangle^{\rho},\langle y\rangle^{\rho}\right\}$, with some $c>0$, but if $|x-y| \geq$ $c \max \left\{\langle x\rangle^{\rho},\langle y\rangle^{\rho}\right\}$, then we deduce (1.4) from (1.3):

$$
f(y) f(x)^{-1} \leq C\langle x\rangle^{m_{-}}\langle y\rangle^{-m} \leq C C_{2}(1+|x-y|)^{N_{2}},
$$

where $N_{2}=2 \max \left\{0,-m, m_{-}\right\} / \rho, \quad C_{2}=\max \left\{1,2 c^{-2 / \rho}\right\}$.

The next lemma describes an especially nice subclass of $\mathcal{F}_{\rho}(f)$. 
Lemma 1.2. For all $j$, let

$$
c_{j}^{0}(W ; f ; \rho):=\sup _{|\alpha| \leq j} \sup _{\mathbb{R}^{n}}\left|D^{\alpha} W(x)\right| f(x)^{-1}\langle x\rangle^{\rho|\alpha|}<\infty .
$$

Then (1.1) holds.

Proof. Without loss of generality we may assume that $\left\{\alpha^{j}\right\}$ is the canonical basis of $\mathbb{R}^{n}$. Then

$$
\begin{gathered}
\left(\delta_{j} W\right)(x+\gamma)=\int_{0}^{1}\left(\partial_{j} W\right)\left(x+\gamma+t \alpha^{j}\right) d t \\
\left(\delta_{j_{1}} \cdots \delta_{j_{p}} W\right)(x+\gamma)=\int_{0}^{1} \cdots \int_{0}^{1}\left(\partial_{j_{1}} \cdots \partial_{j_{p}} W\right)\left(x+\gamma+t_{1} \alpha^{j_{1}}+\cdots+t_{p} \alpha^{j_{p}}\right) d t_{1} \cdots d t_{p},
\end{gathered}
$$

and due to (1.5)

$$
\begin{gathered}
\left\|\left(\delta_{j_{1}} \cdots \delta_{j_{p}} W\right)(\cdot+\gamma)\right\|_{\text {End } L_{2}} \\
\leq C_{p} \sup _{|x-\gamma| \leq p+1} f(x)\langle x\rangle^{-p \rho} \leq C_{1, p} f(\gamma)\langle\gamma\rangle^{-p \rho}
\end{gathered}
$$

(the last inequality follows from the similar inequality obtained in the proof of Lemma 1.1).

If $W$ satisfies $(1.5)$ we write $W \in \mathcal{F}_{\rho}^{0}(f)$; this is a Fréchet space with $c_{j}^{0}(\cdot ; f ; \rho)$ as seminorms.

1.2. Some definitions of the theory of pdo on a torus. Let $B$ be a Banach space. $L^{-\infty}\left(\mathbb{R}^{n^{*}} / \Gamma^{*} ; B\right)$ stands for a class of integral operators whose kernels are of the class $C^{\infty}\left(\mathbb{R}^{n^{*}} / \Gamma^{*} \times \mathbb{R}^{n^{*}} / \Gamma^{*} ; B\right)$.

Let $f$ satisfy (1.2)-(1.3), and set $\Lambda(x)=\langle x\rangle$. We say that $a \in S_{\rho, 0}\left(f ; \mathbb{R}^{n} ; B\right)$ if and only if

$n_{m}(a ; f ; \rho):=\sup _{|\alpha|+|\beta| \leq m} \sup _{\mathbb{R}^{2 n}}\left\|D_{\xi}^{\alpha} D_{x}^{\beta} a(x, \xi)\right\|_{B} f(-\xi)^{-1}\langle\xi\rangle^{\rho|\alpha|}<\infty, \quad m=0,1, \ldots$

It is a Fréchet space with $n_{m}(\cdot ; f ; \rho)$ as seminorms. We set

$$
S^{-\infty}\left(\mathbb{R}^{n} ; B\right):=\bigcap_{m} S_{\rho, 0}\left(\Lambda^{m} ; \mathbb{R}^{n} ; B\right)
$$

If $A$ is a pdo with symbol of the class $S_{\rho, 0}\left(f ; \mathbb{R}^{n} ; B\right)$, we write $A \in L_{\rho, 0}\left(f ; \mathbb{R}^{n} ; B\right)$.

We say that $A \in L_{\rho, 0}\left(f ; \mathbb{R}^{n^{*}} / \Gamma^{*} ; B\right)$ if and only if the following two conditions hold

a) for any $\phi, \psi \in C^{\infty}\left(\mathbb{R}^{n^{*}} / \Gamma^{*}\right)$ with $\operatorname{supp} \phi \cap \operatorname{supp} \psi=\emptyset$,

$$
\phi A \psi \in L^{-\infty}\left(\mathbb{R}^{n^{*}} / \Gamma^{*} ; B\right) ;
$$

b) for a local chart $\kappa: U \rightarrow U^{1}$ and any $\phi, \psi \in C_{0}^{\infty}\left(U^{1}\right)$,

$$
\phi A \psi=\left(\kappa^{-1}\right)^{*}(\phi \circ \kappa) A_{\kappa}(\psi \circ \kappa) \kappa^{*},
$$

where $A_{\kappa} \in L_{\rho, 0}\left(f ; \mathbb{R}^{n} ; B\right)$.

The symbol $a_{\kappa}$ of $A_{\kappa}$ is called a local symbol of $A$. Under the conditions imposed on the local charts, local symbols give rise to the global symbol $a$, which is a well-defined function on $T^{*}\left(\mathbb{R}^{n^{*}} / \Gamma^{*}\right)$ modulo symbols of the class $S^{-\infty}$. We write $a \in S_{\rho, 0}\left(f ; \mathbb{R}^{n^{*}} / \Gamma^{*} ; B\right)$.

If $B=\mathbb{C}$, one omits $B$ in all the notation.

For further information about pdo of these classes, see Section 5. 
1.3. Calculation of the symbol of $\mathcal{W}$. We want to show that $\mathcal{W}$ belongs to $L_{\rho, 0}\left(f ; \mathbb{R}^{n^{*}} / \Gamma^{*} ;\right.$ End $\left.L_{2}(\Omega)\right)$ and calculate its symbol. In doing so, we may use $\phi, \psi \in C^{\infty}\left(\mathbb{R}^{n^{*}} / \Gamma^{*}\right)$ with sufficiently small diam $\operatorname{supp} \phi$, diam $\operatorname{supp} \psi$.

Lemma 1.3. Let $\phi, \psi \in C^{\infty}\left(\mathbb{R}^{n^{*}} / \Gamma^{*}\right)$ satisfy the following condition: there are $j \in\{1, \ldots, n\}$ and $c>0$ such that

$$
\left|\exp \left\{-i\left(\theta^{\prime}-\theta, \alpha^{j}\right)\right\}-1\right|>c, \quad \forall \theta \in \operatorname{supp} \phi, \theta^{\prime} \in \operatorname{supp} \psi .
$$

Then $\phi \mathcal{W} \psi \in L^{-\infty}\left(\mathbb{R}^{n^{*}} / \Gamma^{*} ;\right.$ End $\left.L_{2}(\Omega)\right)$.

Proof. Take $\theta \in \operatorname{supp} \phi$ and $j$ from (1.6). We have

$$
\begin{gathered}
\sum_{\gamma \in \Gamma} \int_{\mathbb{R}^{n^{*}} / \Gamma^{*}} W(\cdot+\gamma) \exp \left\{i\left(\theta^{\prime}-\theta, \gamma\right)\right\} \psi\left(\theta^{\prime}\right) u\left(\cdot, \theta^{\prime}\right) d \theta^{\prime} \\
=\sum_{\gamma \in \Gamma} \int_{\mathbb{R}^{n^{*}} / \Gamma^{*}} W\left(\cdot+\gamma+\alpha^{j}\right) \exp \left\{i\left(\theta^{\prime}-\theta, \gamma+\alpha^{j}\right)\right\} \psi\left(\theta^{\prime}\right) u\left(\cdot, \theta^{\prime}\right) d \theta^{\prime} \\
=\sum_{\gamma \in \Gamma} \int_{\mathbb{R}^{n^{*}} / \Gamma^{*}} W(\cdot+\gamma) \exp \left\{i\left(\theta^{\prime}-\theta, \gamma\right)\right\} \exp \left\{i\left(\theta^{\prime}-\theta, \alpha^{j}\right)\right\} \psi\left(\theta^{\prime}\right) u\left(\cdot, \theta^{\prime}\right) d \theta^{\prime} \\
+\sum_{\gamma \in \Gamma} \int_{\mathbb{R}^{n^{*}} / \Gamma^{*}}\left(\delta_{j} W\right)(\cdot+\gamma) \exp \left\{i\left(\theta^{\prime}-\theta, \gamma\right)\right\} \exp \left\{i\left(\theta^{\prime}-\theta, \alpha^{j}\right)\right\} \psi\left(\theta^{\prime}\right) u\left(\cdot, \theta^{\prime}\right) d \theta^{\prime} .
\end{gathered}
$$

By substituting $W(\cdot+\gamma)\left(1-\exp \left\{i\left(\theta^{\prime}-\theta, \alpha^{j}\right)\right\}\right)^{-1}$ for $W(\cdot+\gamma)$, we obtain

$$
\begin{aligned}
\sum_{\gamma \in \Gamma} \int_{\mathbb{R}^{n^{*}} / \Gamma^{*}} W(\cdot+\gamma) \exp \left\{i\left(\theta^{\prime}-\theta, \gamma\right)\right\} \psi\left(\theta^{\prime}\right) u\left(\cdot, \theta^{\prime}\right) d \theta^{\prime} \\
=\sum_{\gamma \in \Gamma} \int_{\mathbb{R}^{n^{*}} / \Gamma^{*}}\left(\exp \left\{-i\left(\theta^{\prime}-\theta, \alpha^{j}\right)\right\}-1\right)^{-1}\left(\delta_{j} W\right)(\cdot+\gamma) \\
\quad \times \exp \left\{i\left(\theta^{\prime}-\theta, \gamma\right)\right\} \psi\left(\theta^{\prime}\right) u\left(\cdot, \theta^{\prime}\right) d \theta^{\prime} .
\end{aligned}
$$

Iterating this procedure of "summation by parts" we get, for any $N$,

$$
\begin{aligned}
\sum_{\gamma \in \Gamma} \int_{\mathbb{R}^{n^{*}} / \Gamma^{*}} W(\cdot+\gamma) \exp \left\{i\left(\theta^{\prime}-\theta, \gamma\right)\right\} \psi\left(\theta^{\prime}\right) u\left(\cdot, \theta^{\prime}\right) d \theta^{\prime} \\
=\sum_{\gamma \in \Gamma} \int_{\mathbb{R}^{n^{*}} / \Gamma^{*}}\left(\exp \left\{-i\left(\theta^{\prime}-\theta, \alpha^{j}\right)\right\}-1\right)^{-N}\left(\delta_{j}^{N} W\right)(\cdot+\gamma) \\
\quad \times \exp \left\{i\left(\theta^{\prime}-\theta, \gamma\right)\right\} \psi\left(\theta^{\prime}\right) u\left(\cdot, \theta^{\prime}\right) d \theta^{\prime} .
\end{aligned}
$$

On the support of the integrand, the first factor is bounded due to (1.6), and the second one admits an estimate

$$
\left\|\left(\delta_{j}^{N} W\right)(\cdot+\gamma)\right\| \leq C_{N}\langle\gamma\rangle^{-m-\rho N}, \quad \forall \gamma \in \Gamma,
$$

due to (1.1). Take $N>(n-m) / \rho$ and $u \in C^{\infty}\left(\mathbb{R}^{n^{*}} / \Gamma^{*} ; L_{2}(\Omega)\right)$; then we can do the summation first to see that $\phi \mathcal{W} \psi$ is an integral operator with the kernel

$$
\begin{gathered}
K\left(W, \phi, \psi ; \theta, \theta^{\prime}\right) \\
=\frac{1}{\operatorname{vol} \Omega^{*}} \sum_{\gamma \in \Gamma}\left(\exp \left\{-i\left(\theta^{\prime}-\theta, \alpha^{j}\right)\right\}-1\right)^{-N} \phi(\theta) \psi\left(\theta^{\prime}\right)\left(\delta_{j}^{N} W\right)(\cdot+\gamma) \exp \left\{i\left(\theta^{\prime}-\theta, \gamma\right)\right\} .
\end{gathered}
$$


Hence

$$
\left\|K\left(W, \phi, \psi ; \theta, \theta^{\prime}\right)\right\|_{\operatorname{End} L_{2}(\Omega)} \leq C(W, \phi, \psi) \sum_{\gamma \in \Gamma}\langle\gamma\rangle^{-\rho N-m}=C_{1}(W, \phi, \psi)
$$

uniformly in $\theta, \theta^{\prime}$.

To obtain the similar estimate for a derivative $D_{\theta}^{\alpha} D_{\theta^{\prime}}^{\beta} K\left(W, \phi, \psi ; \theta, \theta^{\prime}\right)$, we take $N>(n-m+|\alpha|+|\beta|) / \rho$ and differentiate (1.7).

Lemma 1.4. Let $\kappa: U \rightarrow U^{1}$ be a local chart with small $\operatorname{diam} U$, and let $\phi, \psi \in$ $C_{0}^{\infty}\left(U^{1}\right)$.

Then

$$
\phi \mathcal{W} \psi=\left(\kappa^{-1}\right)^{*}(\phi \circ \kappa)\left(B_{\kappa, 0}+B_{\kappa, \infty}\right)(\psi \circ \kappa) \kappa^{*},
$$

where $B_{\kappa, 0} \in L_{\rho, 0}\left(f ; \mathbb{R}^{n} ;\right.$ End $\left.L_{2}(\Omega)\right)$ has the symbol $b_{\kappa, 0}=b_{\kappa, 0}(\xi)$, which is independent of $\theta$, and $B_{\kappa, \infty} \in L^{-\infty}\left(\mathbb{R}^{n} ;\right.$ End $\left.L_{2}(\Omega)\right)$.

Proof. Fix $\mu \in C_{0}^{\infty}(\mathbb{R}), 0 \leq \mu \leq 1, \mu(t)=1(|t|<1), \mu(t)=0(|t|>2)$, and for $M>1$, set

$$
W_{M}(x)=\mu(|x| / M) W(x), \quad W_{-M}=W-W_{M}, \quad \mathcal{W}_{ \pm M}=\mathcal{U} W_{ \pm M} \mathcal{U}^{*} .
$$

Then $W_{ \pm M}$ satisfies (1.1) uniformly in $M>1$. Take $u \in C^{\infty}\left(\mathbb{R}^{n^{*}} / \Gamma^{*} ; L_{2}(\Omega)\right)$. Integration by parts gives

$$
\begin{aligned}
& \left(\mathcal{W}_{-M} \psi u\right)(\cdot, \theta) \\
& =\frac{1}{\operatorname{vol} \Omega^{*}} \sum_{\gamma \in \Gamma} W_{-M}(\cdot+\gamma) \int_{\mathbb{R}^{n^{*}} / \Gamma^{*}} \exp \left\{i\left(\theta^{\prime}-\theta, \gamma\right)\right\}\left(1+|\gamma|^{2}\right)^{-N} \\
& \quad \times\left(1-\Delta_{\theta^{\prime}}\right)^{N} \psi\left(\theta^{\prime}\right) u\left(x, \theta^{\prime}\right) d \theta^{\prime} .
\end{aligned}
$$

Take $N>n-m$; since $|x| \geq M$ for $x \in \operatorname{supp} W_{-M}$, we have

$$
\sup _{\theta \in \mathbb{R}^{n^{*}} / \Gamma^{*}}\left\|\left(\mathcal{W}_{-M} \psi u\right)(\cdot, \theta)\right\|_{L_{2}(\Omega)} \leq C(W, N) \sum_{|\gamma| \geq M}\langle\gamma\rangle^{-m-N} \rightarrow 0,
$$

as $M \rightarrow \infty$. To obtain similar estimates for $D_{\theta}^{\alpha}\left(\mathcal{W}_{-M} \psi u\right)$, we take $N>|\alpha|+n-m$. Thus, $\mathcal{W}_{-M} u \rightarrow 0$ in $C^{\infty}\left(\mathbb{R}^{n^{*}} / \Gamma^{*} ; L_{2}(\Omega)\right)$ as $M \rightarrow \infty$.

It follows that (1.8) will be proved together with the formula

$$
\phi \mathcal{W}_{M} \psi=\left(\kappa^{-1}\right)^{*}(\phi \circ \kappa)\left(B_{\kappa, M, 0}+B_{\kappa, M, \infty}\right)(\psi \circ \kappa) \kappa^{*},
$$

where

(a) $B_{\kappa, M, 0} \in L_{\rho, 0}\left(f ; \mathbb{R}^{n} ;\right.$ End $\left.L_{2}(\Omega)\right)$ and $B_{\kappa, M, \infty} \in L^{-\infty}\left(\mathbb{R}^{n} ;\right.$ End $\left.L_{2}(\Omega)\right)$ uniformly in $M$,

(b) $B_{\kappa, M, 0}$ has the symbol $b_{\kappa, M, 0}=b_{\kappa, M, 0}(\xi)$ which is independent of $\theta$, and

(c) The limits

$$
\begin{aligned}
b_{\kappa, 0}(\xi) & =\lim _{M \rightarrow \infty} b_{\kappa, M, 0}(\xi), \\
b_{\kappa, \infty}(\theta, \xi) & =\lim _{M \rightarrow \infty} b_{\kappa, \infty, 0}(\theta, \xi) .
\end{aligned}
$$

exists uniformly on any compact set in $\mathbb{R}^{n}$ and $U \times \mathbb{R}^{n}$ respectively. 
Take $\chi_{1}, \chi_{2} \in C_{0}^{\infty}(U)$ such that $(\phi \circ \kappa) \chi_{1}=\phi \circ \kappa,(\psi \circ \kappa) \chi_{2}=\psi \circ \kappa, \chi_{2}(\theta)=1$ for $\theta \in \operatorname{supp} \chi_{1}$, and put

$$
=\frac{1}{\operatorname{vol} \Omega^{*}} \sum_{\gamma \in \Gamma} W_{M}(\cdot+\gamma) \int_{\mathbb{R}^{n}} \chi_{1}(\theta) \chi_{2}\left(\theta^{\prime}\right) \exp \left\{i\left(\theta^{\prime}-\theta, \gamma\right)\right\} u\left(x, \theta^{\prime}\right) d \theta^{\prime} .
$$

This is an integral operator with the kernel

$$
K\left(W_{M}, \chi_{1}, \chi_{2} ; \theta, \theta^{\prime}\right)=\frac{1}{\operatorname{vol} \Omega^{*}} \sum_{\gamma \in \Gamma} W_{M}(\cdot+\gamma) \chi_{1}(\theta) \chi_{2}\left(\theta^{\prime}\right) \exp \left\{i\left(\theta^{\prime}-\theta, \gamma\right)\right\} .
$$

Therefore, $\tilde{W}_{M}$ is a pdo with the symbol

$$
\begin{aligned}
\tilde{b}_{M}(\theta, \xi)=\int_{\mathbb{R}^{n}} \chi_{1}(\theta) \chi_{2}\left(\theta+\theta^{\prime}\right) K\left(W_{M}, \chi_{1}, \chi_{2} ; \theta, \theta+\theta^{\prime}\right) \exp \left\{i\left(\theta^{\prime}, \xi\right)\right\} d \theta^{\prime} \\
=\frac{1}{\operatorname{vol} \Omega^{*}} \int_{\mathbb{R}^{n}} \chi_{1}(\theta) \chi_{2}\left(\theta+\theta^{\prime}\right) \sum_{\gamma \in \Gamma} W_{M}(\cdot+\gamma) \exp \left\{i\left(\theta^{\prime}, \gamma+\xi\right)\right\} d \theta^{\prime}
\end{aligned}
$$

Take $\chi \in C_{0}^{\infty}\left(\mathbb{R}^{n}\right)$ such that $\chi\left(\theta^{\prime}\right)=1$ on the support of the integrand in (1.12), notice that

where

$$
\chi_{1}(\theta) \chi_{2}\left(\theta+\theta^{\prime}\right)=\chi_{1}(\theta)+r\left(\theta, \theta^{\prime}\right),
$$

$$
\left|D_{\theta}^{\nu} D_{\theta^{\prime}}^{\omega} r\left(\theta, \theta^{\prime}\right)\right| \leq C(\nu, \omega, N)\left|\theta^{\prime}\right|^{N},
$$

for all $N$ and all multi-indices $\nu, \omega$, and set

$$
\begin{gathered}
b_{M, 0}(\xi)=\frac{1}{\operatorname{vol} \Omega^{*}} \int_{\mathbb{R}^{n}} \chi\left(\theta^{\prime}\right) \sum_{\gamma \in \Gamma} W_{M}(\cdot+\gamma) \exp \left\{i\left(\theta^{\prime}, \gamma+\xi\right)\right\} d \theta^{\prime}, \\
\tilde{b}_{M, 0}(\theta, \xi)=\chi_{1}(\theta) b_{M, 0}(\xi), \\
\tilde{b}_{M, \infty}(\theta, \xi)=\frac{1}{\operatorname{vol} \Omega^{*}} \int_{\mathbb{R}^{n}} r\left(\theta, \theta^{\prime}\right) \chi\left(\theta^{\prime}\right) \sum_{\gamma \in \Gamma} W_{M}(\cdot+\gamma) \exp \left\{i\left(\theta^{\prime}, \gamma+\xi\right)\right\} d \theta^{\prime} .
\end{gathered}
$$

Clearly,

$$
\tilde{b}_{M}(\theta, \xi)=\tilde{b}_{M, 0}(\theta, \xi)+\tilde{b}_{M, \infty}(\theta, \xi) .
$$

Integration by parts gives

$$
\begin{gathered}
\left(\xi^{\sigma} D_{\theta}^{\alpha} D_{\xi}^{\beta} \tilde{b}_{M, \infty}\right)(\theta, \xi) \\
=\frac{1}{\operatorname{vol} \Omega^{*}} \int_{\mathbb{R}^{n}} D_{\theta}^{\alpha} r\left(\theta, \theta^{\prime}\right) \chi\left(\theta^{\prime}\right) \sum_{\gamma \in \Gamma} W_{M}(\cdot+\gamma) \theta^{\prime^{\beta}} \exp \left\{i\left(\theta^{\prime}, \gamma\right)\right\} D_{\theta^{\prime}}^{\sigma} \exp \left\{i\left(\theta^{\prime}, \xi\right)\right\} d \theta^{\prime} \\
=\frac{1}{\operatorname{vol} \Omega^{*}} \int_{\mathbb{R}^{n}}(-1)^{|\sigma|} D_{\theta^{\prime}}^{\sigma}\left(D_{\theta}^{\alpha} r\left(\theta, \theta^{\prime}\right) \chi\left(\theta^{\prime}\right) \theta^{\prime^{\beta}}\right. \\
\left.\times \exp \left\{i\left(\theta^{\prime}, \gamma\right)\right\}\right) \sum_{\gamma \in \Gamma} W_{M}(\cdot+\gamma) \exp \left\{i\left(\theta^{\prime}, \xi\right)\right\} d \theta^{\prime} .
\end{gathered}
$$

The estimate (1.13) allows us to sum by parts as in Lemma 1.3 and obtain

$$
\left\|\xi^{\sigma} D_{\theta}^{\alpha} D_{\xi}^{\beta} \tilde{b}_{M, \infty}(\theta, \xi)\right\|_{\text {End } L_{2}(\Omega)} \leq C(\sigma, \alpha, \beta) \sum_{\gamma \in \Gamma}\langle\gamma\rangle^{-n-1}=C_{1}(\sigma, \alpha, \beta),
$$


uniformly in $\theta, \xi ;$ similarly, for $M_{1}>M>1$,

$$
\left\|\xi^{\sigma} D_{\theta}^{\alpha} D_{\xi}^{\beta}\left(\tilde{b}_{M, \infty}(\theta, \xi)-\tilde{b}_{M_{1}, \infty}(\theta, \xi)\right)\right\|_{\text {End } L_{2}(\Omega)} \leq C(\sigma, \alpha, \beta) \sum_{|\gamma| \geq M}\langle\gamma\rangle^{-n-1} \rightarrow 0
$$

as $M \rightarrow \infty$, uniformly in $\theta, \xi$ from a given compact.

Hence, $\tilde{b}_{M, \infty} \in S^{-\infty}\left(\mathbb{R}^{n} ;\right.$ End $\left.L_{2}(\Omega)\right)$ uniformly in $M>1$, and the limit (1.11) exists with $b_{\infty} \in S^{-\infty}\left(\mathbb{R}^{n} ;\right.$ End $\left.L_{2}(\Omega)\right)$.

Now we consider

$$
D_{\xi}^{\beta} b_{M, 0}(\xi)=\frac{1}{\operatorname{vol} \Omega^{*}} \int_{\mathbb{R}^{n}} \chi\left(\theta^{\prime}\right) \sum_{\gamma \in \Gamma} W_{M}(\cdot+\gamma) \theta^{\prime^{\beta}} \exp \left\{i\left(\theta^{\prime}, \gamma+\xi\right)\right\} d \theta^{\prime}
$$

Set

$$
\delta^{\beta}=\delta_{1}^{\beta_{1}} \cdots \delta_{n}^{\beta_{n}}, \quad f_{\beta}\left(\theta^{\prime}\right)=\prod_{1 \leq j \leq n}\left(\left(\exp \left\{-i\left(\theta_{j}^{\prime}, a^{j}\right)\right\}-1\right)^{-1} \theta_{j}^{\prime}\right)^{\beta_{j}} \chi\left(\theta^{\prime}\right),
$$

sum by parts and integrate by parts:

$$
\begin{aligned}
D_{\xi}^{\beta} b_{M, 0}(\xi) & \\
= & \frac{1}{\operatorname{vol} \Omega^{*}} \int_{\mathbb{R}^{n}}\left(\left(1-\Delta_{\theta^{\prime}}\right)^{N_{1}} f_{\beta}\left(\theta^{\prime}\right)\right) \\
& \times \sum_{\gamma \in \Gamma}\left(\delta^{\beta} W_{M}\right)(\cdot+\gamma)\langle\gamma+\xi\rangle^{-2 N_{1}} \exp \left\{i\left(\theta^{\prime}, \gamma+\xi\right)\right\} d \theta^{\prime} .
\end{aligned}
$$

Let $N$ be as in (1.4). Then due to (1.1) and (1.4),

$$
\begin{gathered}
\left\|\left(\delta^{\beta} W_{M}\right)(\cdot+\gamma)\langle\gamma+\xi\rangle^{-2 N_{1}}\right\| \leq C_{\beta, N_{1}} f(\gamma)\langle\gamma\rangle^{-\rho|\beta|}\langle\gamma+\xi\rangle^{-2 N_{1}} \\
\leq C_{1, \beta, N_{1}} f(-\xi)\langle\gamma+\xi\rangle^{N-2 N_{1}}\langle\gamma\rangle^{-\rho|\beta|} \leq C_{2, \beta, N_{1}} f(-\xi)\langle\xi\rangle^{-\rho|\beta|}\langle\gamma+\xi\rangle^{N+\rho|\beta|-2 N_{1}} .
\end{gathered}
$$

Taking $N_{1}>(n+\rho|\beta|+N) / 2$, we obtain

$$
\left\|D_{\xi}^{\beta} b_{M, 0}(\xi)\right\|_{\text {End } \left.L_{2}(\Omega)\right)} \leq C_{1}(\beta, N) f(-\xi)\langle\xi\rangle^{-\rho|\beta|} .
$$

Similarly, for $M_{1}>M>1$,

$$
\left\|D_{\xi}^{\beta}\left(b_{M, 0}(\xi)-b_{M_{1}, 0}(\xi)\right)\right\|_{\left.\operatorname{End} L_{2}(\Omega)\right)} \leq C(\beta, N) \sum_{|\gamma|>M} f(\gamma)\langle\gamma\rangle^{-\rho|\beta|}\langle\gamma+\xi\rangle^{-2 N_{1}} \rightarrow 0
$$

as $M \rightarrow \infty$, uniformly in $\xi$ from a given compact.

Hence, $b_{M, 0} \in S_{\rho, 0}\left(f ; \mathbb{R}^{n} ;\right.$ End $\left.L_{2}(\Omega)\right)$ uniformly in $M>1$, and the limit (1.10) exists with $b_{0} \in S_{\rho, 0}\left(f ; \mathbb{R}^{n} ;\right.$ End $\left.L_{2}(\Omega)\right)$.

This completes the proof of Lemma 1.3.

\section{Lemma 1.5. Put}

$$
w(\xi)=\lim _{M \rightarrow \infty} \lim _{t \rightarrow \infty} \frac{1}{\operatorname{vol} \Omega^{*}} \int_{\mathbb{R}^{n}} \sum_{\gamma \in \Gamma} \mu\left(\left|\theta^{\prime}\right| / t\right) W_{M}(\cdot+\gamma) \exp \left\{i\left(\theta^{\prime}, \gamma+\xi\right)\right\} d \theta^{\prime} .
$$

Then $b_{0}-w \in S^{-\infty}\left(\mathbb{R}^{n} ;\right.$ End $\left.L_{2}(\Omega)\right)$.

Proof. The arguments from Lemma 1.4 show that

$$
b_{0}(\xi)=\lim _{M \rightarrow \infty} \frac{1}{\operatorname{vol} \Omega^{*}} \int_{\mathbb{R}^{n}} \chi\left(\theta^{\prime}\right) \sum_{\gamma \in \Gamma} W_{M}(\cdot+\gamma) \exp \left\{i\left(\theta^{\prime}, \gamma+\xi\right)\right\} d \theta^{\prime} .
$$


Hence

$$
\begin{gathered}
w(\xi)-b_{0}(\xi) \\
=\lim _{M \rightarrow \infty} \lim _{t \rightarrow \infty} \frac{1}{\operatorname{vol} \Omega^{*}} \int_{\mathbb{R}^{n}}\left(\mu\left(\left|\theta^{\prime}\right| / t\right)-\chi\left(\theta^{\prime}\right)\right) \sum_{\gamma \in \Gamma} W_{M}(\cdot+\gamma) \exp \left\{i\left(\theta^{\prime}, \gamma+\xi\right)\right\} d \theta^{\prime} .
\end{gathered}
$$

By integrating by parts, we obtain

$$
\begin{gathered}
w(\xi)-b_{0}(\xi) \\
=\lim _{M \rightarrow \infty} \lim _{t \rightarrow \infty} \frac{1}{\operatorname{vol} \Omega^{*}} \int_{\mathbb{R}^{n}} \sum_{\gamma \in \Gamma}\left(1+|\gamma+\xi|^{2}\right)^{-N} W_{M}(\cdot+\gamma) \exp \left\{i\left(\theta^{\prime}, \gamma+\xi\right)\right\} \\
\times\left(1-\Delta_{\theta^{\prime}}\right)^{N}\left(\mu\left(\left|\theta^{\prime}\right| / t\right)-\chi\left(\theta^{\prime}\right)\right) d \theta^{\prime} \\
=\lim _{M \rightarrow \infty} \lim _{t \rightarrow \infty} \frac{1}{\operatorname{vol} \Omega^{*}} \int_{\mathbb{R}^{n}} \sum_{\gamma \in \Gamma}\left(1+|\gamma+\xi|^{2}\right)^{-N} W_{M}(\cdot+\gamma) \exp \left\{i\left(\theta^{\prime}, \gamma+\xi\right)\right\} \\
+\lim _{M \rightarrow \infty} \lim _{t \rightarrow \infty} \frac{1}{\operatorname{vol} \Omega^{*}} \int_{\mathbb{R}^{n}} \sum_{\gamma \in \Gamma}\left(1+|\gamma+\xi|^{2}\right)^{-N} W_{M}(\cdot+\gamma) \exp \left\{i\left(\theta^{\prime}, \gamma+\xi\right)\right\} \\
\times\left\{\left(1-\Delta_{\theta^{\prime}}\right)^{N}-1\right\}\left(-\chi\left(\theta^{\prime}\right)\right) d \theta^{\prime} \\
\times \lim _{M \rightarrow \infty} \lim _{t \rightarrow \infty} \frac{1}{\operatorname{vol} \Omega^{*}} \int_{\mathbb{R}^{n}} \sum_{\gamma \in \Gamma}\left(1+|\gamma+\xi|^{2}\right)^{-N} W_{M}(\cdot+\gamma) \exp \left\{i\left(\theta^{\prime}, \gamma+\xi\right)\right\} \\
\times\left\{\left(1-\Delta_{\theta^{\prime}}\right)^{N}-1\right\}\left(\mu\left(\left|\theta^{\prime}\right| / t\right) d \theta^{\prime} .\right.
\end{gathered}
$$

Fix $\xi$ and $N>(n-m) /(2 \rho)$; then all the integrals converge absolutely and are uniformly bounded. Passing to the limit under the untegral sign, we obtain

$$
\begin{gathered}
w(\xi)-b_{0}(\xi) \\
=\lim _{M \rightarrow \infty} \frac{1}{\operatorname{vol} \Omega^{*}} \int_{\mathbb{R}^{n}} \sum_{\gamma \in \Gamma}\left(1+|\gamma+\xi|^{2}\right)^{-N} W_{M}(\cdot+\gamma) \exp \left\{i\left(\theta^{\prime}, \gamma+\xi\right)\right\} \times \\
\times\left(1-\Delta_{\theta^{\prime}}\right)^{N}\left(1-\chi\left(\theta^{\prime}\right)\right) d \theta^{\prime} .
\end{gathered}
$$

Since $1-\chi\left(\theta^{\prime}\right)=0$ in a neighbourhood of 0 , we can sum by parts and prove that $w-b_{0} \in S^{-\infty}\left(\mathbb{R}^{n} ;\right.$ End $\left.L_{2}(\Omega)\right)$.

By combining Lemmas 1.3-1.5, we obtain the following theorem.

Theorem 1.6. $\mathcal{W} \in L_{\rho, 0}\left(f ; \mathbb{R}^{n^{*}} / \Gamma^{*} ;\right.$ End $\left.L_{2}(\Omega)\right)$, and its symbol is given by $(1.14)$. 
1.4. A formula for $w$ in the case $W \in \mathcal{F}_{\rho}^{0}(f)$. Define the formal power series $L_{j} \in \mathbb{C}[[s, t]]=\mathbb{C}\left[\left[s_{1}, \ldots, s_{n}, t_{1}, \ldots, t_{n}\right]\right]$ by

$$
L_{j}(s, t)=t_{j}\left(\exp \left\{i s_{j}\right\}-1\right) /\left(\exp \left\{i t_{j}\right\}-1\right),
$$

and set

$$
\begin{gathered}
L=\left(L_{1}, \ldots, L_{n}\right), \quad L^{1}(s, t)=s-L(s, t), \\
L^{k}(s, t)=L^{k-1}(s, L(s, t)), \quad k=2,3, \ldots .
\end{gathered}
$$

Since $L_{j}(s, t) \in \sum_{l=1}^{n} s_{l} \mathbb{C}[[s, t]]$, there exists the limit

$$
L^{\infty}(s)=\lim _{k \rightarrow \infty} L^{k}(s, t) .
$$

Set

$$
\hat{L}(D)=\frac{1}{\operatorname{vol} \Omega} \int_{\Omega}\left(1-\exp \left\{i\left(x, L^{\infty}(-D)\right)\right\}\right) d x \in \mathbb{C}\left[\left[D_{1}, \ldots, D_{n}\right]\right] .
$$

Since $L_{j}(s, t)=s_{j}+$ terms of order greater than 1 , we have $L^{1}(s, t)=0 \bmod s$, and $\hat{L}(D)=I+$ terms of order greater than 1 .

Theorem 1.7. Let $W \in \mathcal{F}_{\rho}^{0}(f)$. Then

$$
w(\xi) \sim \sum_{|\alpha|+s \geq 0} \frac{(\cdot)^{\alpha}}{\alpha !}\left(\hat{L}(D)^{s}(i D)^{\alpha} W\right)(-\xi),
$$

where

$$
(\cdot)^{\alpha}: L_{2}(\Omega) \ni u(x) \mapsto x^{\alpha} u(x) \in L_{2}(\Omega) .
$$

The asymptotic sum in (1.16) is understood in the usual sense: let

$$
\sum_{|\alpha|+s \geq 0} \frac{(\cdot)^{\alpha}}{\alpha !} \hat{L}(D)^{s}(i D)^{\alpha}=\sum_{|\beta| \geq 0} c_{\beta}(\cdot) D^{\beta},
$$

then (1.16) means that, for any $N$,

$$
w(\xi)=\sum_{|\beta|<N} c_{\beta}(\cdot)\left(D^{\beta} W\right)(-\xi)+r_{N}(\xi),
$$

where $r_{N} \in S_{\rho, 0}\left(f \Lambda^{-N \rho} ; \mathbb{R}^{n^{*}} / \Gamma^{*} ;\right.$ End $\left.L_{2}(\Omega)\right)$. In particular,

$$
w(\xi)=W(-\xi)+\sum_{j=1}^{n}(\cdot)_{j}\left(i D_{j} W\right)(-\xi)+r_{2}(\xi) .
$$

Proof. The argument used in the proof of Lemma 1.4 shows that it suffices to prove (1.17) for $W \in \mathbb{C}_{0}^{\infty}\left(\mathbb{R}^{n}\right)$, provided for any $j$

$$
n_{j}\left(r_{N} ; f \Lambda^{-\rho N} ; \rho\right) \leq C c_{l}^{0}(W ; f ; \rho),
$$

where $C, l$ depend on $j, \rho, f, N$, and $n$, but not on $W$.

Due to Lemma 1.5 (see (1.15)), we have modulo $S^{-\infty}\left(\mathbb{R}^{n} ;\right.$ End $L_{2}(\Omega)$ ):

$$
\begin{gathered}
w(\xi)=\frac{1}{\operatorname{vol} \Omega^{*}} \int_{\mathbb{R}^{n}} d \theta^{\prime} \chi\left(\theta^{\prime}\right) \sum_{\gamma \in \Gamma} W(\cdot+\gamma) \exp \left\{i\left(\theta^{\prime}, \gamma+\xi\right)\right\} \\
=\sum_{|\alpha|<N} \frac{(\cdot)^{\alpha}}{\alpha !} w_{\alpha}(\xi)+\sum_{|\alpha|=N} \frac{(\cdot)^{\alpha}}{\alpha !} r_{\alpha}(\xi)
\end{gathered}
$$


where

$$
\begin{gathered}
w_{\alpha}(\xi)=\frac{1}{\operatorname{vol} \Omega^{*}} \int_{\mathbb{R}^{n}} d \theta^{\prime} \chi\left(\theta^{\prime}\right) \sum_{\gamma \in \Gamma}\left((i D)^{\alpha} W\right)(\gamma) \exp \left\{i\left(\theta^{\prime}, \gamma+\xi\right)\right\}, \\
r_{\alpha}(\xi)=\frac{1}{\operatorname{vol} \Omega^{*}} \int_{\mathbb{R}^{n}} d \theta^{\prime} \chi\left(\theta^{\prime}\right) \int_{0}^{1} d t \sum_{\gamma \in \Gamma}\left((i D)^{\alpha} W\right)(t(\cdot)+\gamma) \exp \left\{i\left(\theta^{\prime}, \gamma+\xi\right)\right\} .
\end{gathered}
$$

In (1.20) and below, "modulo $S^{-\infty}$ " has a meaning similar to (1.19), namely, that $S^{-\infty}$-seminorms of an "error term" admit estimates via seminorms of $W \in \mathcal{F}_{\rho}^{0}(f)$.

Due to $(1.5),\left((i D)^{\alpha} W\right)(t(\cdot)+\gamma) \in \mathcal{F}_{\rho}\left(f \Lambda^{-|\alpha| \rho}\right)$ uniformly in $t \in[0,1]$. Therefore Theorem 1.6 gives

$$
r_{\alpha} \in S_{\rho, 0}\left(f \Lambda^{-|\alpha| \rho} ; \mathbb{R}^{n^{*}} / \Gamma^{*} ; \text { End } L_{2}(\Omega)\right)
$$

with $r_{\alpha}$ satisfying (1.19).

For $V \in C_{0}^{\infty}\left(\mathbb{R}^{n}\right)$, set $V_{1}(x)=V(-x)$. We have

$$
\begin{gathered}
\frac{1}{\operatorname{vol} \Omega^{*}} \int_{\mathbb{R}^{n}} d \theta^{\prime} \chi\left(\theta^{\prime}\right) \sum_{\gamma \in \Gamma} V(\gamma) \exp \left\{i\left(\theta^{\prime}, \xi+\gamma\right)\right\} \\
=\frac{1}{\operatorname{vol} \Omega^{*}} \int_{\mathbb{R}^{n}} d \theta^{\prime} \chi\left(\theta^{\prime}\right) \sum_{\gamma \in \Gamma} V_{1}(\gamma) \exp \left\{i\left(\theta^{\prime}, \xi-\gamma\right)\right\} \\
=v_{1}(\xi)+v_{2}(\xi),
\end{gathered}
$$

where

$$
\begin{gathered}
v_{1}(\xi)=\frac{1}{\operatorname{vol} \Omega^{*}} \int_{\mathbb{R}^{n}} d \theta^{\prime} \chi\left(\theta^{\prime}\right) \sum_{\gamma \in \Gamma} \frac{1}{\operatorname{vol} \Omega} \int_{\Omega} d x V_{1}(\gamma+x) \exp \left\{i\left(\theta^{\prime}, \xi-\gamma-x\right)\right\}, \\
v_{2}(\xi)=\frac{1}{\operatorname{vol} \Omega^{*}} \int_{\mathbb{R}^{n}} d \theta^{\prime} \chi\left(\theta^{\prime}\right) \sum_{\gamma \in \Gamma} \frac{1}{\operatorname{vol} \Omega} \int_{\Omega} d x \times \\
\times\left(V_{1}(\gamma)-V_{1}(\gamma+x) \exp \left\{-i\left(\theta^{\prime}, x\right)\right\}\right) \exp \left\{i\left(\theta^{\prime}, \xi-\gamma\right)\right\} .
\end{gathered}
$$

Clearly, modulo $S^{-\infty}\left(\mathbb{R}^{n}\right)$,

$$
\begin{gathered}
v_{1}(\xi)=(2 \pi)^{-n} \int_{\mathbb{R}^{n}} d \theta^{\prime} \chi\left(\theta^{\prime}\right) \int_{\mathbb{R}^{n}} d x V_{1}(x) \exp \left\{i\left(\theta^{\prime}, \xi-x\right)\right\} \\
=V_{1}(\xi)=V(-\xi)
\end{gathered}
$$

and

$$
\begin{gathered}
v_{2}(\xi) \sim \frac{1}{\operatorname{vol} \Omega^{*}} \int_{\mathbb{R}^{n}} d \theta^{\prime} \chi\left(\theta^{\prime}\right) \sum_{\gamma \in \Gamma} \frac{1}{\operatorname{vol} \Omega} \int_{\Omega} d x \\
\times\left(\left(I-\exp \left\{i\left(x, D_{x}-\theta^{\prime}\right)\right\}\right) V_{1}\right)(\gamma) \exp \left\{i\left(\theta^{\prime}, \xi-\gamma\right)\right\},
\end{gathered}
$$

the RHS in (1.24) being understood in the sense explained in Section 5.

Write the summation by parts formula in the form

$$
\sum_{\gamma \in \Gamma} V_{1}(\gamma) \theta^{\omega} \exp \{-i(\theta, \gamma)\}=\sum_{\gamma \in \Gamma}\left(L(D, \theta)^{\omega} V_{1}\right)(\gamma) \exp \{-i(\theta, \gamma)\}
$$

where

$$
L(D, \theta)^{\omega}=L_{1}(D, \theta)^{\omega_{1}} \cdots L_{n}(D, \theta)^{\omega_{n}},
$$


Set

$$
L_{j}(D, \theta)=\theta_{j}\left(\exp \left\{i D_{j}\right\}-1\right) /\left(\exp \left\{i \theta_{j}\right\}-1\right) .
$$

$$
L^{1}(s, t)=s-L(s, t), \quad L^{k}(s, t)=L^{k-1}(s, L(s, t)), k=2,3, \ldots .
$$

By substituting (1.25) into (1.24), we obtain

$$
\begin{gathered}
v_{2}(\xi) \sim \frac{1}{\operatorname{vol} \Omega^{*}} \int_{\mathbb{R}^{n}} d \theta^{\prime} \chi(\xi) \sum_{\gamma \in \Gamma} \frac{1}{\operatorname{vol} \Omega} \int_{\Omega} d x \\
\times\left(\left(I-\exp \left\{i\left(x, L^{1}(D, \theta)\right)\right\}\right) V_{1}\right)(\gamma) \exp \left\{i\left(\theta^{\prime}, \xi-\gamma\right)\right\} .
\end{gathered}
$$

By iterating this procedure and using Theorem 5.6, we obtain (1.26) with $L^{k}$ instead of $L^{1}, k=2,3, \ldots$. Since $L_{l}(s, t) \in \sum_{j=1}^{n} s_{j} \mathbb{C}[[s, t]]$, we have

$$
v_{2}(\xi) \sim \frac{1}{\operatorname{vol} \Omega^{*}} \int_{\mathbb{R}^{n}} d \theta^{\prime} \chi(\xi) \sum_{\gamma \in \Gamma}\left(\tilde{L}(D) V_{1}\right)(\gamma) \exp \left\{i\left(\theta^{\prime}, \xi-\gamma\right)\right\}
$$

where

$$
\tilde{L}(D)=\frac{1}{\operatorname{vol} \Omega} \int_{\Omega}\left(I-\exp \left\{i\left(x, L^{\infty}(D)\right)\right\}\right) d x .
$$

It follows from (1.22), (1.27) and (1.23), that

$$
\frac{1}{\operatorname{vol} \Omega^{*}} \int_{\mathbb{R}^{n}} d \theta^{\prime} \chi\left(\theta^{\prime}\right) \sum_{\gamma \in \Gamma}\left((I-\tilde{L}(D)) V_{1}\right)(\gamma) \exp \left\{i\left(\theta^{\prime}, \xi-\gamma\right)\right\} \sim V_{1}(\xi) .
$$

Replacing $V_{1}$ in (1.28) by $\sum_{j \geq 0} \tilde{L}(D)^{j} V_{1}$ and using Theorem 5.5, we obtain

$$
\begin{gathered}
\frac{1}{\operatorname{vol} \Omega^{*}} \int_{\mathbb{R}^{n}} d \theta^{\prime} \chi\left(\theta^{\prime}\right) \sum_{\gamma \in \Gamma} V(\gamma) \exp \left\{i\left(\theta^{\prime}, \xi+\gamma\right)\right\} \\
\sim \sum_{j \geq 0}\left(\tilde{L}(D)^{j} V_{1}\right)(\xi)=\sum_{j \geq 0}\left(\hat{L}(D)^{j} V\right)(-\xi) .
\end{gathered}
$$

We apply (1.29) with $V=(i D)^{\alpha} W$ to (1.20); then (1.20) and (1.21) give (1.16).

\section{Asymptotics of $N_{-}(t ; H-E ; W)$}

2.1. Auxiliary variational lemmas. We shall need the following definition and lemmas.

Let $\mathfrak{A}$ be a quadratic form in a Hilbert space $\mathfrak{H}$, with a domain $D(\mathfrak{A})$, and let $V \subset D(\mathfrak{A})$ be a subspace. We assume that $\mathfrak{A}$ is closable, and we set

$$
\mathcal{N}(\lambda, \mathfrak{A}, V)=\sup \left\{\operatorname{dim} L \mid L \subset V, \mathfrak{A}[u]<\lambda\|u\|_{\mathfrak{H}}^{2} \quad \forall(0 \neq) u \in L\right\} .
$$

For $A$ an operator in $\mathfrak{H}$ and $\mathfrak{A}[u]=\langle A u, u\rangle_{\mathfrak{H}}, D(\mathfrak{A})=D(A)$, write $\mathcal{N}(\lambda, A, V)$ instead of $\mathcal{N}(\lambda, \mathfrak{A}, V)$.

For $A$ a self-adjoint operator and $(a, b) \subset \mathbb{R}$, set $N((a, b) ; A)=\operatorname{dim} \operatorname{Ran} P_{(a, b)}(A)$, where $P_{(a, b)}(A)$ is a spectral projection of $A$. Set also $N(\lambda ; A)=N((-\infty, \lambda) ; A)$.

The following lemmas are well-known and widely used; for proofs, see e.g. $[6$, $16,18]$.

Lemma 2.1. Let $A$ be a semibounded from below self-adjoint operator with the domain $D(A)$.

Then $N(\lambda ; A)=\mathcal{N}(\lambda ; A ; D(A))$. 
Lemma 2.2. $\mathcal{N}(\lambda ; \mathfrak{A} ; V)$ is independent of $V$ provided $V$ is a core of the form $\mathfrak{A}$.

Lemma 2.3. If $\mathfrak{A}[u] \leq \mathfrak{A}_{1}[u] \quad \forall u \in V$, then

$$
\mathcal{N}\left(\lambda ; \mathfrak{A}_{1} ; V\right) \leq \mathcal{N}(\lambda ; \mathfrak{A} ; V) .
$$

Lemma 2.4. If $V \subset V_{1}$, then

$$
\mathcal{N}(\lambda ; \mathfrak{A} ; V) \leq \mathcal{N}\left(\lambda ; \mathfrak{A} ; V_{1}\right) .
$$

Lemma 2.5. Let $H_{s}$ be a Hilbert space, $\mathfrak{A}_{s}$ a quadratic form in $H_{s}, V_{s} \subset D\left(\mathfrak{A}_{s}\right)$ a subspace $(s=0,1, \ldots, r)$, let

$$
l: \bigoplus_{1 \leq s \leq r} V_{s} \rightarrow V_{0}
$$

be an isomorphism, and let

$$
\mathfrak{A}_{0}\left[l\left(u_{1}, \ldots, u_{r}\right)\right]=\sum_{s=1}^{r} \mathfrak{A}_{s}\left[u_{s}\right] .
$$

Then

$$
\mathcal{N}\left(\lambda ; \mathfrak{A}_{0} ; V_{0}\right)=\sum_{s=1}^{r} \mathcal{N}\left(\lambda ; \mathfrak{A}_{s} ; V_{s}\right) .
$$

2.2. Proof of Theorem 0.2. The Birman-Schwinger principle implies that $N_{ \pm}(t ; H-E ; W)$ is equal to the number of positive eigenvalues counted, with multiplicity, of a problem

$$
u= \pm \mu W^{1 / 2}(H-E)^{-1} W^{1 / 2} u, \quad u \in L_{2}\left(\mathbb{R}^{n}\right),
$$

belonging to $(0, t)$ (see $[1,9])$. Let $\mathcal{U}$ be an isometry defined by $(0.3)$, and set $\mathcal{H}=\mathcal{U} H \mathcal{U}^{*}, \mathcal{W}=\mathcal{U} W \mathcal{U}^{*}$. Then we can deal with the problem

$$
u= \pm \mu \mathcal{W}^{1 / 2}(\mathcal{H}-E)^{-1} \mathcal{W}^{1 / 2} u
$$

instead, and Lemmas 2.1, 2.2 and 2.5 give

$$
N_{ \pm}(t ; H-E ; W)=\mathcal{N}\left(0 ; \mathcal{W}^{-1} \mp t(\mathcal{H}-E)^{-1} ; C^{\infty}\left(\mathbb{R}^{n^{*}} / \Gamma^{*} ; L_{2}(\Omega)\right)\right) .
$$

Denote by $\mathcal{P}_{1}(\theta)$ a spectral projection $\mathcal{P}_{(-\infty, E)}(\mathcal{H}(\theta))$, and set $\mathcal{P}_{2}(\theta)=I-\mathcal{P}_{1}(\theta)$. We have $\mathcal{P}_{j} \in C^{\infty}\left(\mathbb{R}^{n^{*}} / \Gamma^{*} ;\right.$ End $\left.L_{2}(\Omega)\right)$. For $j=1,2$, define an orthoprojection $\mathcal{P}_{j}$ in $L_{2}\left(\mathbb{R}^{n^{*}} / \Gamma^{*} ; L_{2}(\Omega)\right)$ by

$$
\left(\mathcal{P}_{j} u\right)(x, \theta)=\mathcal{P}_{j}(\theta) u(x, \theta),
$$

and set $\mathcal{L}_{j}=\mathcal{P}_{j}\left(C^{\infty}\left(\mathbb{R}^{n^{*}} / \Gamma^{*} ; L_{2}(\Omega)\right)\right)$.

It follows from (2.3) and Lemma 2.4 that

$$
N_{-}(t ; H-E ; W) \geq \mathcal{N}\left(0 ; \mathcal{W}^{-1}+t(\mathcal{H}-E)^{-1} ; \mathcal{L}_{1}\right),
$$

which is the estimate from below in Theorem 0.2.

To obtain the estimate from above, we note that it follows from (1.18) and Theorem 5.8 that

$$
\left[\mathcal{W}^{-1}, \mathcal{P}_{j}\right] \in L_{\rho, 0}\left(W^{-1} \Lambda^{-\rho} ; \mathbb{R}^{n^{*}} / \Gamma^{*} ; \text { End } L_{2}(\Omega)\right) ;
$$

therefore, for $u \in C^{\infty}\left(\mathbb{R}^{n^{*}} / \Gamma^{*} ; L_{2}(\Omega)\right)$,

$$
\left\langle\mathcal{W}^{-1} u, u\right\rangle=\sum_{j=1,2}\left\langle\mathcal{W}^{-1} u_{j}, u_{j}\right\rangle+\left\langle K u_{1}, u_{2}\right\rangle,
$$


where $u_{j}=\mathcal{P}_{j} u$ and $K \in L_{\rho, 0}\left(W^{-1} \Lambda^{-\rho} ; \mathbb{R}^{n^{*}} / \Gamma^{*} ;\right.$ End $\left.L_{2}(\Omega)\right)$.

Set

$$
W_{r, s}(x)=W(x)^{r}\langle x\rangle^{s}, \quad \mathcal{W}_{r, s}=\mathcal{U} W_{r, s} \mathcal{U}^{*}
$$

By Theorem 1.6, $\mathcal{W}_{r, s} \in L_{\rho, 0}\left(W_{r, s} ; \mathbb{R}^{n^{*}} / \Gamma^{*} ;\right.$ End $\left.L_{2}(\Omega)\right)$; therefore Theorem 5.8 gives

$$
K_{1}:=\mathcal{W}_{1 / 2,0} K \in L_{\rho, 0}\left(W_{-1 / 2,-\rho} ; \mathbb{R}^{n^{*}} / \Gamma^{*} ; \text { End } L_{2}(\Omega)\right) .
$$

For any $\epsilon>0$, there exists $C_{\epsilon}>0$ such that

$\left|\left\langle K u_{1}, u_{2}\right\rangle\right| \leq\left\|K_{1} u_{1}\right\|\left\|\mathcal{W}_{-1 / 2,0} u_{2}\right\| \leq \epsilon\left\langle\mathcal{W}_{-1 / 2,0}^{*} \mathcal{W}_{-1 / 2,0} u_{2}, u_{2}\right\rangle+C_{\epsilon}\left\langle K_{1}^{*} K_{1} u_{1}, u_{1}\right\rangle$.

By construction, $\mathcal{W}_{-1 / 2,0}^{*} \mathcal{W}_{-1 / 2,0}=\mathcal{W}^{-1}$; therefore

$$
\left\langle\mathcal{W}^{-1} u, u\right\rangle \geq\left\langle\left(\mathcal{W}^{-1}(1-\epsilon) u_{2}, u_{2}\right\rangle+\left\langle\left(\mathcal{W}^{-1}-C_{\epsilon} K_{1}^{*} K_{1}\right) u_{1}, u_{1}\right\rangle .\right.
$$

Clearly,

$$
\left\langle(\mathcal{H}-E)^{-1} u, u\right\rangle=\sum_{j=1,2}\left\langle(\mathcal{H}-E)^{-1} u_{j}, u_{j}\right\rangle
$$

whence

$$
\left\langle\left(\mathcal{W}^{-1}+t(\mathcal{H}-E)^{-1}\right) u, u\right\rangle
$$

$\geq\left\langle\left(\mathcal{W}^{-1}(1-\epsilon)+t \mathcal{P}_{2}(\mathcal{H}-E)^{-1}\right) u_{2}, u_{2}\right\rangle+\left\langle\left(\mathcal{W}^{-1}-C_{\epsilon} K_{1}^{*} K_{1}+t \mathcal{P}_{1}(\mathcal{H}-E)^{-1}\right) u_{1}, u_{1}\right\rangle$, and we deduce from (2.3) and Lemmas 2.5, 2.3 that

$$
\begin{gathered}
N_{-}(t ; H-E ; W) \leq \mathcal{N}\left(0 ; \mathcal{W}^{-1}(1-\epsilon)+t \mathcal{P}_{2}(\mathcal{H}-E)^{-1} ; \mathcal{L}_{2}\right) \\
+\mathcal{N}\left(0 ; \mathcal{W}^{-1}-C_{\epsilon} K_{1}^{*} K_{1}+t \mathcal{P}_{1}(\mathcal{H}-E)^{-1} ; \mathcal{L}_{1}\right)
\end{gathered}
$$

Fix $\epsilon \in(0,1)$. Then $\mathcal{W}^{-1}(1-\epsilon)$ is positive definite, and since $\mathcal{P}_{2}(\mathcal{H}-E)^{-1} \geq 0$, the first term in the right-hand side of (2.5) is zero. Now Theorems 5.8 and 5.9 give

$$
K_{2}:=\mathcal{W}_{1 / 2, \rho} K_{1}^{*} K_{1} \mathcal{W}_{1 / 2, \rho} \in L_{\rho, 0}\left(1 ; \mathbb{R}^{n^{*}} / \Gamma^{*} ; \text { End } L_{2}(\Omega)\right),
$$

and by Theorem $5.10, K_{2}$ is bounded in $L_{2}\left(\mathbb{R}^{n^{*}} / \Gamma^{*} ; L_{2}(\Omega)\right)$. Hence, there exists $C>0$ such that

$$
-C_{\epsilon} K_{1}^{*} K_{1} \geq-C \mathcal{W}_{-1,-2 \rho},
$$

and due to Lemma 2.3 , we can replace $-C_{\epsilon} K_{1}^{*} K_{1}$ in $(2.5)$ by $-C \mathcal{W}_{-1,-2 \rho}$. Thus,

$$
N_{-}(t ; H-E ; W) \leq \mathcal{N}\left(0 ; \mathcal{W}^{-1}-C \mathcal{W}_{-1,-2 \rho}+t(\mathcal{H}-E)^{-1} ; \mathcal{L}_{1}\right),
$$

which is the estimate from above in Theorem 0.2.

Theorem 0.2 is proved.

2.3. Proof of Theorem 0.3. By applying Theorems 1.7 and 5.8, we obtain for local symbols of $\mathcal{W}_{11, C}^{-1}$ a representation (0.6) with

$$
w_{11, U, \chi}^{-1,-1} \in S_{\rho, 0}\left(W_{-1,-\rho}: \mathbb{R}^{n} ; \text { End } \mathbb{C}^{p}\right)
$$

and the tail belonging to $S_{\rho, 0}\left(W_{-1,-2 \rho} ; \mathbb{R}^{n} ;\right.$ End $\left.\mathbb{C}^{p}\right)$. This means that conditions (6.4)-(6.8) of Theorem 6.4 hold with

$M=\mathbb{R}^{n^{*}} / \Gamma^{*}, \quad f(\xi)=W(-\xi)^{-1}, \quad \mu_{j}(\theta, \xi)=\left(E-E_{j}(\theta)\right) W(-\xi)^{-1}, j=1, \ldots, p$, and $(0.8)$ is imposed in both Theorems 6.4 and 0.3 . Hence, all the conditions of Theorem 6.4 hold, and Theorem 0.2 together with (6.10) gives (0.9).

Theorem 0.3 is proved. 
2.4. Proof of Theorem 0.4. If $W$ admits a full asymptotic expansion

$$
W(x)=W_{-m}(x)+W_{-m-1}(x)+\cdots, \quad \text { as } \quad|x| \rightarrow+\infty,
$$

then Theorem 0.2 is applicable with $\rho=1$. By applying Theorems 1.7 and 5.8, we find that $\mathcal{W}_{11, C}^{-1}$ is an elliptic classical pdo with the principal symbol having eigenvalues

$$
\mu_{j}(\theta, \xi)=\left(E_{j}(\theta)-E\right) W_{-m}(-\xi)^{-1} .
$$

Well-known results for elliptic pdo (see e.g. [11, 19]) give

$$
N\left(t ; \overline{\mathcal{W}_{11, C}^{-1}}\right)=c_{-}\left(H-E ; W_{-m}\right) t^{n / m}+O\left(t^{(n-1) / m}\right),
$$

with the constant in the $O$-term depending on $C$.

Thus, under an additional assumption Theorem 0.4 is proved, and the proof in the general case is reduced to this special one as follows. Let $\tilde{W} \in C^{\infty}\left(\mathbb{R}^{n} ; \mathbb{R}_{+}\right)$be a function which coincides with $W_{-m}$ outside the unit ball. Then due to (0.10), there exists $C_{1}>0$ such that

$$
\tilde{W}_{C_{1}}^{-1}:=\tilde{W}^{-1}-C_{1}\langle\cdot\rangle^{m-1} \leq W^{-1} \leq W_{-C_{1}}^{-1}:=\tilde{W}^{-1}+C_{1}\langle\cdot\rangle^{m-1} .
$$

Set $\tilde{\mathcal{W}}_{ \pm C_{1}}^{-1}=\mathcal{U} \tilde{W}_{ \pm C_{1}}^{-1} \mathcal{U}^{*}$. Using Lemmas 2.1-2.3 and (2.8), and arguing as at the beginning of the proof of Theorem 0.2 , we obtain

$$
\begin{aligned}
\mathcal{N}\left(0 ; \tilde{\mathcal{W}}_{-C_{1}}^{-1}+\right. & \left.t(\mathcal{H}-E)^{-1} ; C^{\infty}\left(\mathbb{R}^{n^{*}} / \Gamma^{*} ; L_{2}(\Omega)\right)\right) \\
& \leq N_{-}(t ; H-E ; W) \\
\leq \mathcal{N}\left(0 ; \tilde{\mathcal{W}}_{C_{1}}^{-1}+\right. & \left.t(\mathcal{H}-E)^{-1} ; C^{\infty}\left(\mathbb{R}^{n^{*}} / \Gamma^{*} ; L_{2}(\Omega)\right)\right) .
\end{aligned}
$$

The functions $\tilde{W}_{ \pm C_{1}}^{-1}$ satisfy the conditions

$$
\begin{gathered}
\left|\partial^{\alpha} \tilde{W}_{ \pm C_{1}}^{-1}(x)\right| \leq C_{\alpha}\langle x\rangle^{m-|\alpha|}, \quad \forall \alpha, \\
\tilde{W}_{ \pm C_{1}}^{-1} \geq c_{2}\langle x\rangle^{m} \quad \text { for }|x| \geq C_{2},
\end{gathered}
$$

where $c_{2}>0$. Therefore we can argue as in the proof of Theorem 0.2 and obtain

$$
N\left(t ; \overline{\tilde{\mathcal{W}}_{11,-C_{1}}^{-1}}\right) \leq \mathcal{N}\left(0 ; \tilde{\mathcal{W}}_{-C_{1}}^{-1}+t(\mathcal{H}-E)^{-1} ; C^{\infty}\left(\mathbb{R}^{n^{*}} / \Gamma^{*} ; L_{2}(\Omega)\right)\right)
$$

$$
N\left(t ; \overline{\tilde{\mathcal{W}}_{11, C_{2}}^{-1}}\right) \geq \mathcal{N}\left(0 ; \tilde{\mathcal{W}}_{C_{1}}^{-1}+t(\mathcal{H}-E)^{-1} ; C^{\infty}\left(\mathbb{R}^{n^{*}} / \Gamma^{*} ; L_{2}(\Omega)\right)\right),
$$

with some $C_{2}>0$. Here $\overline{\tilde{\mathcal{W}}_{11, \pm C_{1}}^{-1}}$ are constructed in the way $\overline{\mathcal{W}_{11, C}^{-1}}$ were constructed.

By applying Theorems 1.7 and 5.6 we find that $\tilde{\mathcal{W}}_{11, C}^{-1}$ is an elliptic classical pdo with principal symbol having eigenvalues $(2.6)$. Hence, for $N\left(t ; \tilde{\mathcal{W}}_{11, C}^{1}\right)$ the asymptotic formula (2.7) holds.

Now, (2.7) and (2.9)-(2.11) give (0.11), and Theorem 0.4 is proved. 
2.5. Proof of Theorem 0.5. If $W$ admits a full asymptotic expansion then $W$ satisfies conditions (0.1) and (0.2) with $m=m_{-}, \rho=1$, and $W^{-1}$ admits the asymptotic expansion

$$
W^{-1}(x)=W_{-m}^{-1}(x)-W_{-m}^{-2}(x) W_{-m-1}(x)+\cdots .
$$

Hence, Theorems 1.7 and 5.6 say that $\mathcal{W}_{11, C}^{-1}$ is a classical pdo with the first two terms of the asymptotic expansion of the symbol given by (0.13) and (0.14). The principal symbol being positive, we may add a smoothing operator (an operator of order $-\infty$ ) and obtain a positive definite operator (this leaves both the principal and the next term of asymptotics unchanged); after that, we can consider the counting function of $1 / m$-th power of the (closure of the) operator under consideration. The principal and subprincipal symbols of this power are given by $(0.15)$ and (0.16), and the results of [11] give (0.17) (modulo the reservations mentioned before Theorem 0.5).

Thus, Theorem 0.5 is proved provided $W$ admits a full asymptotic expansion. In the general case we set

$$
\tilde{W}_{C}^{-1}=W_{-m}^{-1}-W_{-m}^{-2} W_{-m-1}-C\langle\cdot\rangle^{m-1-\epsilon},
$$

notice that due to $(0.12)$ there exists $C>0$ such that

$$
\tilde{W}_{C}^{-1} \leq W^{-1} \leq \tilde{W}_{-C}^{-1},
$$

and reduce the proof to the one for the special case considered above as in the proof of Theorem 0.4.

\section{The Asymptotics of $N_{+}(t ; H-E ; W)$}

3.1. Proof of Theorem 0.6. Define an isometry $\mathcal{U}_{0}: L_{2}\left(\mathbb{R}^{n^{*}} / \Gamma^{*} ; L_{2}(\Omega)\right) \rightarrow$ $L_{2}\left(\mathbb{R}^{n^{*}} / \Gamma^{*} ; L_{2}\left(\mathbb{R}^{n} / \Gamma\right)\right)$ by $u(x, \theta) \mapsto \exp \{-i(\theta, x)\} u(x, \theta)$, and set

$$
\mathcal{H}_{1}=\mathcal{U}_{0} \mathcal{H U}_{0}^{*}
$$

It is multiplication by the operator-valued function

$$
\mathcal{H}_{1}(\theta)=(i \nabla-\theta)^{*}(i \nabla-\theta)+V: H^{2}\left(\mathbb{R}^{n} / \Gamma\right) \rightarrow L_{2}\left(\mathbb{R}^{n} / \Gamma\right) .
$$

Set $\mathcal{W}_{1}=\mathcal{U}_{0} \mathcal{W} \mathcal{U}_{0}^{*}$. It acts as follows:

$$
\left(\mathcal{W}_{1} u\right)(x, \theta)=\frac{1}{\operatorname{vol} \Omega^{*}} \sum_{\gamma \in \Gamma} W_{1}(x+\gamma) \int_{\Omega^{*}} \exp \left\{i\left(\theta^{\prime}-\theta, x+\gamma\right)\right\} u\left(x, \theta^{\prime}\right) d \theta^{\prime},
$$

and trivial modifications of the proofs of Theorems 1.6 and 1.7 give

Theorem 3.1. Let $W$ satisfy (1.1). Then $\mathcal{W}_{1} \in L_{\rho, 0}\left(f ; \mathbb{R}^{n^{*}} / \Gamma^{*} ;\right.$ End $\left.L_{2}\left(\mathbb{R}^{n} / \Gamma\right)\right)$, and if $W$ satisfies (1.5) then its symbol admits a decomposition

$$
w_{1}(\xi)=W(-\xi)+r(\xi),
$$

where $r \in S_{\rho, 0}\left(f \Lambda^{-\rho} ; \mathbb{R}^{n^{*}} / \Gamma^{*} ;\right.$ End $\left.L_{2}\left(\mathbb{R}^{n} / \Gamma\right)\right)$.

Thus, we can use $\mathcal{W}_{1}, \mathcal{H}_{1}$ almost as we used $\mathcal{W}, \mathcal{H}$ before, the difference being that $\mathcal{H}_{1}$ is not a smooth function on $\mathbb{R}^{n^{*}} / \Gamma^{*}$ but all the $\mathcal{H}_{1}(\theta)$ have the same domain; note that $\mathcal{H}_{1}$ is a smooth function on $\overline{\Omega^{*}}$.

To simplify the notation, we use labels $\mathcal{W}, \mathcal{H}$ for $\mathcal{W}_{1}, \mathcal{H}_{1}$. 
Lemma 3.2. Let $V \in L_{\infty}\left(\mathbb{R}^{n}\right)$ and let $E$ be in a gap of $H=-\Delta+V$.

Then there exists $C_{1}>0$ such that

$$
(-\Delta+1)^{-1}-C_{1}(-\Delta+1)^{-3 / 2} \leq(H-E)^{-1} \leq(-\Delta+1)^{-1}+C_{1}(-\Delta+1)^{-3 / 2} .
$$

Proof. Set $B=-\Delta+1, W_{1}=1-E+V$, and consider

$$
T:=(-\Delta+V-E)^{-1}-B^{-1}=B^{-1} W_{1}(-\Delta+V-E)^{-1}=B^{-3 / 4} T_{1} B^{-3 / 4},
$$

where

$$
\begin{gathered}
T_{1}:=B^{-1 / 4} W_{1}(-\Delta+V-E)^{-1} B^{3 / 4} \\
=B^{-1 / 4} W_{1} B^{-1 / 4}+B^{-1 / 4} W_{1} T B^{3 / 4} \\
=B^{-1 / 4} W_{1} B^{-1 / 4}+B^{-1 / 4} W_{1}(\Delta+V-E)^{-1} W_{1} B^{-1 / 4} .
\end{gathered}
$$

We see that $T_{1}$ is bounded in $L_{2}\left(\mathbb{R}^{n}\right)$. Hence there exists $C_{1}>0$ such that

$$
\left|\langle T u, u\rangle_{L_{2}}\right| \leq C_{1}\left\|B^{-3 / 4} u\right\|_{L_{2}}^{2},
$$

and (3.1) follows.

Define an operator $\mathcal{A}$ in $L_{2}\left(\mathbb{R}^{n^{*}} / \Gamma^{*} ; L_{2}\left(\mathbb{R}^{n} / \Gamma\right)\right)$ by

$$
(\mathcal{A} u)(x, \theta)=\left(-\Delta_{x}+1\right) u(x, \theta),
$$

and set $\mathcal{B}=\mathcal{U}_{0} \mathcal{U} B \mathcal{U}^{*} \mathcal{U}_{0}^{*}\left(=\left(i \nabla_{x}-\theta\right)^{2}+1\right)$. For any $s, m \in \mathbb{R}$,

$$
\left(-\Delta_{x}+1\right)^{s}, \quad\left(\left(i \nabla_{x}-\theta\right)^{2}+1\right)^{s}: H^{m}\left(\mathbb{R}^{n} / \Gamma\right) \rightarrow H^{m-2 s}\left(\mathbb{R}^{n} / \Gamma\right)
$$

are bounded (the latter uniformly in $\theta \in \Omega^{*}$ ). Hence there exists $C_{2}>0$ such that $\mathcal{B}^{-3 / 2} \leq C_{2} \mathcal{A}^{-3 / 2}$. Further, similarly to the proof of Lemma 3.2, there exists $C_{3}>0$ such that

$$
\left|\left\langle\left(\mathcal{B}^{-1}-\mathcal{A}^{-1}\right) u, u\right\rangle_{L_{2}}\right| \leq C_{3}\left\langle\mathcal{A}^{-3 / 2} u, u\right\rangle_{L_{2}},
$$

and using (3.1), we find $C>0$ such that

$$
-C \mathcal{A}^{-3 / 2}+\mathcal{A}^{-1} \leq(\mathcal{H}-E)^{-1} \leq \mathcal{A}^{-1}+C \mathcal{A}^{-3 / 2} .
$$

The Birman-Schwinger principle, Lemma 2.3 and (3.2) show that

$$
\begin{gathered}
N\left(0 ; I-t \mathcal{W}^{1 / 2}\left(\mathcal{A}^{-1}-C \mathcal{A}^{-3 / 2}\right) \mathcal{W}^{1 / 2}\right) \leq N_{+}(t ; H-E ; W) \leq \\
\leq N\left(0 ; I-t \mathcal{W}^{1 / 2}\left(\mathcal{A}^{-1}+C \mathcal{A}^{-3 / 2}\right) \mathcal{W}^{1 / 2}\right) .
\end{gathered}
$$

Using Lemma 2.2, we obtain

$$
\begin{gathered}
N\left(0 ; I-t \mathcal{W}^{1 / 2}\left(\mathcal{A}^{-1} \pm C \mathcal{A}^{-3 / 2}\right) \mathcal{W}^{1 / 2}\right) \\
=\mathcal{N}\left(0 ; \mathcal{W}^{-1}-t\left(\mathcal{A}^{-1} \pm C \mathcal{A}^{-3 / 2}\right) ; C^{\infty}\left(\mathbb{R}^{n^{*}} / \Gamma^{*} ; L_{2}\left(\mathbb{R}^{n} / \Gamma\right)\right)\right) .
\end{gathered}
$$

Let $\mathcal{W}^{-1,0}$ be a symmetric positive invertible scalar pdo with the symbol $W(-\xi)^{-1}$, and $\overline{\mathcal{W}^{-1,0}}$ its closure in $L_{2}\left(\mathbb{R}^{n^{*}} / \Gamma^{*}\right)$. Due to $(0.2)$ there exist $C, \rho_{1}>0$ such that

$$
W(x)^{-1}\langle x\rangle^{-\rho} \leq C W(x)^{-1-\rho_{1}} .
$$

Theorem 5.13 shows that $\left(\overline{\mathcal{W}^{-1,0}}\right)^{ \pm\left(1-\rho_{1}\right) / 2} \in L_{\rho, 0}\left(W^{\mp\left(1-\rho_{1}\right) / 2} ; \mathbb{R}^{n^{*}} / \Gamma^{*}\right)$, and Theorems 3.1 and 5.6 give

$$
\left(\overline{\mathcal{W}^{-1,0}}\right)^{-\left(1-\rho_{1}\right) / 2}\left(\mathcal{W}^{-1}-\mathcal{W}_{-1,0}\right)\left(\overline{\mathcal{W}^{-1,0}}\right)^{-\left(1-\rho_{1}\right) / 2} \in L_{\rho, 0}\left(1 ; \mathbb{R}^{n^{*}} / \Gamma^{*}\right) .
$$

By Theorem 5.10, this operator is bounded in $L_{2}\left(\mathbb{R}^{n^{*}} / \Gamma^{*} ; L_{2}\left(\mathbb{R}^{n} / \Gamma\right)\right)$. Hence, there exists $C>0$ such that

$$
\overline{\mathcal{W}^{-1,0}}-C\left(\overline{\mathcal{W}^{-1,0}}\right)^{1-\rho_{1}} \leq \overline{\mathcal{W}^{-1}} \leq \overline{\mathcal{W}^{-1,0}}+C\left(\overline{\mathcal{W}^{-1,0}}\right)^{1-\rho_{1}}
$$


Now Lemma 2.3 and the Birman-Schwinger principle allow us to deduce from (3.3) that

$$
\begin{gathered}
N\left(0 ; \overline{\mathcal{W}^{-1,0}}+C_{2}\left(\overline{\mathcal{W}^{-1,0}}\right)^{1-\rho_{1}}-t\left(\mathcal{A}^{-1}-C \mathcal{A}^{-3 / 2}\right)\right) \\
\leq N_{+}(t ; H-E ; W) \\
\leq N\left(0 ; \overline{\mathcal{W}^{-1,0}}-C_{2}\left(\overline{\mathcal{W}^{-1,0}}\right)^{1-\rho_{1}}-t\left(\mathcal{A}^{-1}+C \mathcal{A}^{-3 / 2}\right)\right)
\end{gathered}
$$

where $\overline{\mathcal{W}^{-1,0}}$ is regarded as an operator in $L_{2}\left(\mathbb{R}^{n^{*}} / \Gamma^{*} ; L_{2}\left(\mathbb{R}^{n} / \Gamma\right)\right)$, with scalar symbol.

It is well-known that the counting function of the spectrum of the Laplacian on $\mathbb{R}^{n} / \Gamma$ has the asymptotics

$$
N(t ;-\Delta)=c_{0} t^{n / 2}+O\left(t^{(n-1) / 2}\right), \quad \text { as } t \rightarrow+\infty
$$

(see, e.g., $[5,19])$, where

$$
c_{0}=(2 \pi)^{-n}\left|v_{n}\right| \operatorname{vol} \Omega \text {. }
$$

Hence, $\lambda_{j}, j=1,2, \ldots$, the eigenvalues of $\left(-\Delta_{x}+1\right)^{-1}$, obey

$$
\lambda_{j}=c_{0}^{2 / n} j^{-2 / n}+O\left(j^{-3 / n}\right), \quad \text { as } j \rightarrow+\infty .
$$

$W$ admits a representation $(0.20),(0.21)$; therefore Theorem 6.4 gives for $\overline{\mathcal{W}^{-1,0}}$, an operator in $L_{2}\left(\mathbb{R}^{n^{*}} / \Gamma^{*}\right)$,

$$
N\left(t ; \overline{\mathcal{W}^{-1,0}}\right)=c_{1}\left(W_{-2}\right) t^{n / 2}+O\left(t^{n / 2-\epsilon_{1}}\right), \quad \text { as } t \rightarrow+\infty,
$$

for some $\epsilon_{1}>0$, where

$$
c_{1}\left(W_{-2}\right)=(2 \pi)^{-n} \operatorname{vol} \Omega^{*} n^{-1} \int_{S_{n-1}} W_{-2}(\xi)^{n / 2} d S(\xi) .
$$

Let $\mu_{1} \leq \mu_{2} \leq \ldots$ be the eigenvalues of an operator $\mathcal{W}^{-1,0}$ in $L_{2}\left(\mathbb{R}^{n^{*}} / \Gamma^{*}\right)$, counted with multiplicity. Then (3.6) implies

$$
\mu_{j}^{-1}=c_{1}\left(W_{-2}\right)^{2 / n} j^{-2 / n}+O\left(j^{-2 / n-\epsilon_{2}}\right), \quad \text { as } j \rightarrow+\infty,
$$

for some $\epsilon_{2}>0$.

Now (3.4) and (3.5) show that there are $C_{2}, C_{3}$ and $C_{4}>0$ such that

$$
\begin{gathered}
\operatorname{card}\{(i, j) \mid i \geq 1, j \geq 1, \\
\left.1<t\left(\left(c_{0} j^{-1}\right)^{2 / n}-C_{2} j^{-3 / n}\right)\left(\left(c_{1}\left(W_{-2}\right) i^{-1}\right)^{2 / n}-C_{3} i^{-2 / n-\epsilon_{2}}\right)\right\}-C_{4} t^{n / 2} \\
\leq N_{+}(t ; H-E ; W) \\
\leq C_{4} t^{n / 2}+\operatorname{card}\{(i, j) \mid i \geq 1, j \geq 1, \\
\left.1<t\left(\left(c_{0} j^{-1}\right)^{2 / n}+C_{2} j^{-3 / n}\right)\left(\left(c_{1}\left(W_{-2}\right) i^{-1}\right)^{2 / n}+C_{3} i^{-2 / n-\epsilon_{2}}\right)\right\} .
\end{gathered}
$$

It is easy to show that

$$
\begin{gathered}
\operatorname{card}\{(i, j) \mid i \geq 1, j \geq 1, \\
\left.1-t\left(\left(c_{0} j^{-1}\right)^{2 / n} \pm C_{2} j^{-3 / n}\right)\left(\left(c_{1}\left(W_{-2}\right) i^{-1}\right)^{2 / n} \pm C_{3} i^{-2 / n-\epsilon_{2}}\right)<0\right\} \\
=\operatorname{meas}\left\{(x, y) \mid x>1, y>1,1-t^{n / 2} c_{0} c_{1}\left(W_{-2}\right) x^{-1} y^{-1}<0\right\}+O\left(t^{n / 2}\right) \\
=\frac{n}{2} c_{0} c_{1}\left(W_{-2}\right) t^{n / 2} \ln t+O\left(t^{n / 2}\right) ;
\end{gathered}
$$

since $\operatorname{vol} \Omega^{*} \operatorname{vol} \Omega=(2 \pi)^{n}$, we have

$$
\frac{n}{2} c_{0} c_{1}\left(W_{-2}\right)=c\left(W_{-2}\right),
$$


where $c\left(W_{-2}\right)$ is given by $(0.23)$, and (3.7) gives $(0.22)$.

This completes the proof of Theorem 0.6.

Note that as far as $V$ was concerned we used only the condition $V \in L_{\infty}\left(\mathbb{R}^{n}\right)$.

3.2. Proof of Theorem 0.8. Due to (2.1) and Lemmas 2.2 and 2.5 we have

$$
N_{+}(t ; H-E ; W)=\mathcal{N}\left(0 ; W^{-1}-t(H-E)^{-1} ; C_{0}^{\infty}\left(\mathbb{R}^{n}\right)\right),
$$

and the right-hand side in (3.8) can be estimated as follows.

Fix $\epsilon>0$ and $\mu \in C_{0}^{\infty}(\mathbb{R})$ such that $0 \leq \mu \leq 1, \mu(t)=1$ for $|t|<1, \mu(t)=0$ for $|t|>2$. For $c \in[1 / 4,2]$, set

$$
\begin{gathered}
\tilde{\mu}_{t, \epsilon, c}(x)=\mu\left(W(x)^{-1} c^{-1} t^{-\epsilon}\right), \quad \tilde{\mu}_{t, \epsilon,-c}=1-\tilde{\mu}_{t, \epsilon, c}, \\
\mu_{t, \epsilon, \pm c}=\tilde{\mu}_{t, \epsilon, \pm c}\left(\sum_{j= \pm c} \tilde{\mu}_{t, \epsilon, j}^{2}\right)^{-1 / 2} \\
W_{t, \epsilon}^{-1}(x)=W(x)^{-1} \tilde{\mu}_{t, \epsilon,-1 / 4}(x)+t^{\epsilon} \tilde{\mu}_{t, \epsilon, 1 / 4} .
\end{gathered}
$$

Due to (0.1) and (0.2),

$$
\begin{gathered}
\left|D^{\alpha} \tilde{\mu}_{t, \epsilon, c}(x)\right| \leq C_{\alpha}\left(|x|+t^{\epsilon / m_{-}}\right)^{-\rho|\alpha|}, \quad \forall \alpha, \\
\left|D^{\alpha} \mu_{t, \epsilon, c}(x)\right| \leq C_{\alpha}\left(|x|+t^{\epsilon / m_{-}}\right)^{-\rho|\alpha|}, \quad \forall \alpha, \\
\left|D^{\alpha} W_{t, \epsilon}^{-1}(x)\right| \leq C_{\alpha} W_{t, \epsilon}^{-1}(x)\left(|x|+t^{\epsilon / m_{-}}\right)^{-\rho|\alpha|}, \quad \forall \alpha .
\end{gathered}
$$

Using (3.9), one obtains

$$
\left[H, \mu_{t, \epsilon, \pm c}\right]=\sum_{1 \leq j \leq n} a_{t, \epsilon, c, j}(x) \partial_{j}+a_{t, \epsilon, c}^{\prime}
$$

where

$$
\left|a_{t, \epsilon, c, j}(x)\right| \leq C t^{-\epsilon / m_{-}}, \quad\left|a_{t, \epsilon, c}^{\prime}(x)\right| \leq C t^{-2 \epsilon / m_{-}} ;
$$

hence for any $E_{0}<\inf \sigma(H)$ there exists $C>0$ such that

$$
\begin{gathered}
\left|\left\langle\left[(H-E)^{-1}, \mu_{t, \epsilon, \pm c}\right] u, u\right\rangle_{L_{2}}\right| \\
=\left|\left\langle\left(H-E_{0}\right)^{1 / 2}(H-E)^{-1}\left[\mu_{t, \epsilon, \pm c}, H\right](H-E)^{-1} u,\left(H-E_{0}\right)^{-1 / 2} u\right\rangle_{L_{2}}\right| \\
\leq C t^{-\epsilon / m_{-}}\left\langle\left(H-E_{0}\right)^{-1} u, u\right\rangle_{L_{2}} .
\end{gathered}
$$

Set $u_{t, \epsilon, \pm 1}=\mu_{t, \epsilon, \pm 1} u$. (3.11) gives

$$
\begin{gathered}
-\left\langle(H-E)^{-1} u, u\right\rangle_{L_{2}}=-\left\langle(H-E)^{-1} \sum_{j= \pm 1} \mu_{t, \epsilon, j}^{2} u, u\right\rangle_{L_{2}} \\
\geq-\sum_{j= \pm 1}\left\langle(H-E)^{-1} u_{t, \epsilon, j}, u_{t, \epsilon, j}\right\rangle_{L_{2}}-C t^{-\epsilon / m_{-}}\left\langle\left(H-E_{0}\right)^{-1} u, u\right\rangle_{L_{2}} .
\end{gathered}
$$

Take $C_{0}>C$ and apply the same procedure to $(H-E)^{-1}-C_{0} t^{-\epsilon / m_{-}}\left(H-E_{0}\right)^{-1}$. The result is

$$
-\left\langle(H-E)^{-1} u, u\right\rangle_{L_{2}} \geq-\sum_{j= \pm 1}\left\langle\left((H-E)^{-1}+C_{0} t^{-\epsilon / m_{-}}\left(H-E_{0}\right)^{-1}\right) u_{t, \epsilon, j}, u_{t, \epsilon, j}\right\rangle_{L_{2}},
$$

for $t>t_{0}$, if $t_{0}$ is sufficiently large. 
Using (3.12) and Lemmas 2.5 and 2.3, we obtain

$$
\begin{gathered}
\mathcal{N}\left(0, W^{-1}-t(H-E)^{-1} ; C_{0}^{\infty}\left(\mathbb{R}^{n}\right)\right) \\
\leq \mathcal{N}\left(0, W^{-1}-t\left((H-E)^{-1}+C_{0} t^{-\epsilon / m_{-}}\left(H-E_{0}\right)^{-1}\right) ; C_{0}^{\infty}\left(\Omega_{t, \epsilon, 5}\right)\right) \\
+\mathcal{N}\left(0, W^{-1}-t\left((H-E)^{-1}+C_{0} t^{-\epsilon / m_{-}}\left(H-E_{0}\right)^{-1}\right) ; C_{0}^{\infty}\left(\Omega_{t, \epsilon,-1}\right)\right),
\end{gathered}
$$

where

$$
\Omega_{t, \epsilon, c}=\left\{x \mid W(x)^{-1}<c t^{\epsilon}\right\}, \quad \Omega_{t, \epsilon,-c}=\left\{x \mid W(x)^{-1}>c t^{\epsilon}\right\} .
$$

On $\Omega_{t, \epsilon,-1}$, we have $W^{-1}=W_{t, \epsilon}^{-1}$; hence we can rewrite (3.13) as

$$
\begin{gathered}
\mathcal{N}\left(0 ; W^{-1}-t(H-E)^{-1} ; C_{0}^{\infty}\left(\mathbb{R}^{n}\right)\right) \\
\leq \mathcal{N}\left(0, W^{-1}-t\left((H-E)^{-1}+C_{0} t^{-\epsilon / m_{-}}\left(H-E_{0}\right)^{-1}\right) ; C_{0}^{\infty}\left(\Omega_{t, \epsilon, 5}\right)\right) \\
+\mathcal{N}\left(0, W_{t, \epsilon}^{-1}-t\left((H-E)^{-1}+C_{0} t^{-\epsilon / m_{-}}\left(H-E_{0}\right)^{-1}\right) ; C_{0}^{\infty}\left(\mathbb{R}^{n}\right)\right) .
\end{gathered}
$$

Similarly, there exists $C_{0}>0$ such that

$$
\begin{gathered}
\mathcal{N}\left(0 ; W_{t, \epsilon}^{-1}-t\left((H-E)^{-1}-C_{0} t^{-\epsilon / m_{-}}\left(H-E_{0}\right)^{-1}\right) ; C_{0}^{\infty}\left(\mathbb{R}^{n}\right)\right) \\
\leq \mathcal{N}\left(0 ; W_{t, \epsilon}^{-1}-t(H-E)^{-1} ; C_{0}^{\infty}\left(\Omega_{t, \epsilon, 5}\right)\right)+\mathcal{N}\left(0 ; W_{t, \epsilon}^{-1}-t(H-E)^{-1} ; C_{0}^{\infty}\left(\Omega_{t, \epsilon,-1}\right)\right) \\
\leq \mathcal{N}\left(0 ; W_{t, \epsilon}^{-1}-t(H-E)^{-1} ; C_{0}^{\infty}\left(\Omega_{t, \epsilon, 5}\right)\right)+\mathcal{N}\left(0 ; W^{-1}-t(H-E)^{-1} ; C_{0}^{\infty}\left(\mathbb{R}^{n}\right)\right) .
\end{gathered}
$$

There exist $C_{1}, c_{1}>0$ such that $W^{-1}(x) \geq c_{1}>0$, and

$$
\begin{gathered}
W^{-1}-t\left((H-E)^{-1}+C_{0} t^{-\epsilon / m_{-}}\left(H-E_{0}\right)^{-1}\right) \geq c_{1}-C_{1} t(-\Delta+1)^{-1}, \\
W_{t, \epsilon}^{-1}-t(H-E)^{-1} \geq c_{1}-C_{1} t(-\Delta+1)^{-1},
\end{gathered}
$$

and Lemma 2.3 gives

$$
\begin{gathered}
\mathcal{N}\left(0 ; W^{-1}-t\left((H-E)^{-1}+C_{0} t^{-\epsilon / m_{-}}\left(H-E_{0}\right)^{-1}\right) ; C_{0}^{\infty}\left(\Omega_{t, \epsilon, 5}\right)\right) \\
\leq \mathcal{N}\left(0 ; c_{1}-C_{1} t(-\Delta+1)^{-1} ; C_{0}^{\infty}\left(\Omega_{t, \epsilon, 5}\right)\right),
\end{gathered}
$$

$$
\mathcal{N}\left(0 ; W_{t, \epsilon}^{-1}-t(H-E)^{-1} ; C_{0}^{\infty}\left(\Omega_{t, \epsilon, 5}\right)\right) \leq \mathcal{N}\left(0 ; c_{1}-C_{1} t(-\Delta+1)^{-1} ; C_{0}^{\infty}\left(\Omega_{t, \epsilon, 5}\right)\right)
$$

But $(-\Delta+1)^{-1}$ is a classical elliptic pdo of order -2 ; hence Theorem 6.2 gives

$$
\begin{gathered}
\mathcal{N}\left(0 ; c_{1}-C_{1} t(-\Delta+1)^{-1} ; C_{0}^{\infty}\left(\Omega_{t, \epsilon, 5}\right)\right) \\
\leq C_{2} \text { meas } \Omega_{t, \epsilon, 6} t^{n / 2} \leq C_{3} t^{n / 2+n \epsilon / m} .
\end{gathered}
$$

(See also [14], where asymptotic formulae for pdo of negative order were derived from Theorem 6.2.) By gathering (3.14)-(3.18) we obtain

$$
\begin{gathered}
-C_{3} t^{n / 2+n \epsilon / m}+\mathcal{N}\left(0 ; W_{t, \epsilon}^{-1}-t\left((H-E)^{-1}-C_{0} t^{-\epsilon / m}-\left(H-E_{0}\right)^{-1}\right) ; C_{0}^{\infty}\left(\mathbb{R}^{n}\right)\right) \\
\leq \mathcal{N}\left(0 ; W^{-1}-t(H-E)^{-1} ; C_{0}^{\infty}\left(\mathbb{R}^{n}\right)\right) \\
\leq C_{3} t^{n / 2+n \epsilon / m}+\mathcal{N}\left(0 ; W_{t, \epsilon}^{-1}-t\left((H-E)^{-1}+C_{0} t^{-\epsilon / m_{-}}\left(H-E_{0}\right)^{-1}\right) ; C_{0}^{\infty}\left(\mathbb{R}^{n}\right)\right) .
\end{gathered}
$$

We choose $\epsilon>0$ so that $n / 2+n \epsilon / m<n / m$; this is possible due to the condition $m \in(0,2)$. 
3.3. Using Lemmas 2.5 and 2.2, we obtain

$$
\begin{gathered}
\mathcal{N}\left(0 ; W_{t, \epsilon}^{-1}-t\left((H-E)^{-1} \pm C_{0} t^{-\epsilon / m_{-}}\left(H-E_{0}\right)^{-1}\right) ; C_{0}^{\infty}\left(\mathbb{R}^{n}\right)\right) \\
=\mathcal{N}\left(0 ; I-t\left(W_{t, \epsilon}^{-1}\right)^{-1 / 2}\left((H-E)^{-1} \pm C_{0} t^{-\epsilon / m_{-}}\left(H-E_{0}\right)^{-1}\right)\left(W_{t, \epsilon}^{-1}\right)^{-1 / 2} ; C_{0}^{\infty}\left(\mathbb{R}^{n}\right)\right) \\
=\mathcal{N}\left(0 ; I-t\left(W_{t, \epsilon}^{-1}\right)^{-1 / 2}\left((H-E)^{-1} \pm C_{0} t^{-\epsilon / m_{-}}\left(H-E_{0}\right)^{-1}\right)\left(W_{t, \epsilon}^{-1}\right)^{-1 / 2} ; L_{2}\left(\mathbb{R}^{n}\right)\right) \\
=\mathcal{N}\left(0 ; I-t\left(\mathcal{W}_{t, \epsilon}^{-1}\right)^{-1 / 2}\left((\mathcal{H}-E)^{-1} \pm C_{0} t^{-\epsilon / m_{-}}\left(\mathcal{H}-E_{0}\right)^{-1}\right)\left(\mathcal{W}_{t, \epsilon}^{-1}\right)^{-1 / 2} ;\right. \\
\left.L_{2}\left(\mathbb{R}^{n^{*}} / \Gamma^{*} ; L_{2}\left(\mathbb{R}^{n} / \Gamma\right)\right)\right) \\
=\mathcal{N}\left(0 ; I-t\left(\mathcal{W}_{t, \epsilon}^{-1}\right)^{-1 / 2}\left((\mathcal{H}-E)^{-1} \pm C_{0} t^{-\epsilon / m_{-}}\left(\mathcal{H}-E_{0}\right)^{-1}\right)\left(\mathcal{W}_{t, \epsilon}^{-1}\right)^{-1 / 2} ;\right. \\
\left.C^{\infty}\left(\mathbb{R}^{n^{*}} / \Gamma^{*} ; L_{2}\left(\mathbb{R}^{n} / \Gamma\right)\right)\right) \\
=\mathcal{N}\left(0 ; \mathcal{W}_{t, \epsilon}^{-1}-t\left((\mathcal{H}-E)^{-1} \pm C_{0} t^{-\epsilon / m_{-}}\left(\mathcal{H}-E_{0}\right)^{-1}\right) ; C^{\infty}\left(\mathbb{R}^{n^{*}} / \Gamma^{*} ; L_{2}\left(\mathbb{R}^{n} / \Gamma\right)\right)\right),
\end{gathered}
$$

where $\mathcal{W}_{t, \epsilon}^{-1}=\mathcal{U} W_{t, \epsilon}^{-1} \mathcal{U}^{*}$.

Due to (3.10), the derivatives of $W_{t, \epsilon}^{-1}$ decay as $t \rightarrow+\infty$. Therefore the same estimates as in Section 2 now give

1) for $\phi, \psi \in C^{\infty}\left(\mathbb{R}^{n^{*}} / \Gamma^{*}\right)$ with $\operatorname{supp} \phi \cap \operatorname{supp} \psi=\emptyset, \psi \mathcal{W}_{t, \epsilon}^{-1} \psi$ is an integral operator with kernel $K_{t, \epsilon}\left(\theta, \theta^{\prime}\right)$ for all $N, \alpha, \beta$,

$$
\left\|D_{\theta}^{\alpha} D_{\theta^{\prime}}^{\beta} K_{t, \epsilon}\left(\theta, \theta^{\prime}\right)\right\|_{\operatorname{End} L_{2}\left(\mathbb{R}^{n} / \Gamma\right) \leq C_{\alpha, \beta, N} t^{-N} ;}
$$

2) for a local chart $\kappa: U \rightarrow U^{1}$ with small $\operatorname{diam} U$ and $\phi, \psi \in C_{0}^{\infty}\left(U^{1}\right)$,

$$
\phi \mathcal{W}_{t, \epsilon}^{-1} \psi=\left(\kappa^{-1}\right)^{*}(\phi \circ \kappa)\left(W_{t, \epsilon}^{-1}(-D)+W_{t, \epsilon}^{-1,-1}\right)(\psi \circ \kappa) \kappa^{*},
$$

where $W_{t, \epsilon}^{-1,-1}$ is a pdo with symbol satisfying the estimates

$$
\left\|D_{\xi}^{\alpha} D_{\theta}^{\beta} w_{t, \epsilon}^{-1,-1}(\theta, \xi)\right\|_{\operatorname{End} L_{2}\left(\mathbb{R}^{n} / \Gamma\right)} \leq C_{\alpha, \beta} t^{-\epsilon / m_{-}} W_{t, \epsilon}^{-1}(-\xi)\left(|\xi|+t^{\epsilon / m_{-}}\right)^{-\rho|\alpha|},
$$

for all $\alpha, \beta$.

Identify $\Omega^{*}$ with $\mathbb{R}^{n^{*}} / \Gamma^{*}$, fix $\epsilon_{1} \in\left(0, \rho \epsilon / 2 m_{-}\right)$, and cut $\Omega^{*}$ into boxes of size $c_{0} t^{-\epsilon_{1}}$ with $1<c_{0}<2$. We set

$$
\begin{gathered}
U_{t, \epsilon, j}^{+}=\left\{x \mid \operatorname{dist}\left(x, U_{t, \epsilon, j}\right)<t^{-2 \epsilon_{1}}\right\}, \\
U_{t, \epsilon, j}^{-}=\left\{x \in U_{t, \epsilon, j} \mid \operatorname{dist}\left(x, \partial U_{t, \epsilon, j}\right)>t^{-2 \epsilon_{1}}\right\},
\end{gathered}
$$

and construct a partition of unity

$$
\sum_{j} \phi_{t, \epsilon, j}^{2}(\theta)=1, \quad \forall \theta \in \mathbb{R}^{n^{*}} / \Gamma^{*}
$$

with the following properties:

$$
\begin{gathered}
\left|D^{\alpha} \phi_{t, \epsilon, j}(\theta)\right| \leq C_{\alpha, \epsilon} t^{2 \epsilon_{1}|\alpha|}, \quad \forall \alpha, \\
0 \leq \phi_{t, \epsilon, j} \leq 1, \quad \phi_{t, \epsilon, j}=1 \text { on } U_{t, \epsilon, j}^{-}, \quad \operatorname{supp} \phi_{t, \epsilon, j} \subset U_{t, \epsilon, j}^{+} .
\end{gathered}
$$

Let $\kappa_{j}: U_{j} \rightarrow U_{j}^{1}$ be local charts such that $\kappa_{j}^{-1} \circ \kappa_{l}$ are restrictions of orthogonal operators and $U_{t, \epsilon, j}^{+} \subset U_{j}$. Consider

$$
K_{t, \epsilon, j}=\left[\mathcal{W}_{t, \epsilon}^{-1}, \phi_{t, \epsilon, j}\right] \phi_{t, \epsilon, j} .
$$


Clearly, (3.21) for $\mathcal{W}_{t, \epsilon}^{-1}$ yields the same estimate for $K_{t, \epsilon, j}$; therefore we may consider $K_{t, \epsilon, j}$ in appropriate local charts. Now, (3.10), (3.23) and (3.25) mean that (in the notation of Section 6)

$$
\begin{gathered}
W_{t, \epsilon}^{-1}(-D) \in \Psi\left(W_{t, \epsilon}^{-1}(\cdot,-\cdot) ; g_{t, \epsilon}\right), \quad W_{t, \epsilon}^{-1,-1} \in \Psi\left(t^{-\epsilon / m_{-}} W_{t, \epsilon}^{-1}(\cdot,-\cdot) ; g_{t, \epsilon}\right), \\
\phi_{t, \epsilon, j} \in \Psi\left(1, g_{t, \epsilon}\right),
\end{gathered}
$$

uniformly in $t \geq 1$, where

$$
g_{t, \epsilon ; \theta, \xi}\left(\theta^{\prime}, \xi^{\prime}\right)=\left(|\xi|+t^{\epsilon / m_{-}}\right)^{-2 \rho}\left|\xi^{\prime}\right|^{2}+t^{4 \epsilon_{1}}\left|\theta^{\prime}\right|^{2}
$$

is a metric on $\mathbb{R}^{2 n}$. One easily verifies that when $\epsilon_{1} \in\left(0, \epsilon \rho / 2 m_{-}\right)$the metric $g_{t, \epsilon}$ is $\sigma$-temperate, $W_{t, \epsilon}^{-1}(\cdot,-\cdot)$ is a $\sigma, g$-temperate function, and

$$
h_{t, \epsilon}(\theta, \xi):=\left(|\xi|+t^{\epsilon / m}\right)^{-\rho} t^{2 \epsilon_{1}} \leq t^{-\epsilon_{2}},
$$

where $\epsilon_{2}=\epsilon \rho / m_{-}-2 \epsilon_{1}>0$. Now Theorem 5.2 gives

$$
K_{t, \epsilon, j} \in \Psi\left(t^{-\epsilon_{2}} W_{t, \epsilon}^{-1}(\cdot,-\cdot) ; g_{t, \epsilon}\right) .
$$

Using once again Theorem 5.2, Theorem 5.13 and (3.21), we obtain for $\psi_{j}=1$ on $\operatorname{supp} \phi_{t, \epsilon, j}$

$$
\begin{gathered}
\left(\overline{\mathcal{W}_{t, \epsilon}^{-1}}\right)^{-1 / 2} K_{t, \epsilon, j}\left(\overline{\mathcal{W}_{t, \epsilon}^{-1}}\right)^{-1 / 2} \\
=t^{-\epsilon_{2}}\left(\kappa_{j}^{-1}\right)^{*}\left(\psi_{j} \circ \kappa_{j}\right) K_{t, \epsilon, j}^{0}\left(\phi_{j} \circ \kappa_{j}\right) \kappa_{j}^{*}+T_{t, \epsilon, j}^{-\infty},
\end{gathered}
$$

where $T_{t, \epsilon, j}^{-\infty}$ satisfies $(3.21)$ and $K_{t, \epsilon, j}^{0} \in \Psi\left(1 ; g_{t, \epsilon}\right)$ uniformly in $t \geq 1$. Theorem 5.4 (the $L_{2}$-boundedness theorem) provides $C>0$ such that

$$
\left\|\left(\overline{\mathcal{W}_{t, \epsilon}^{-1}}\right)^{-1 / 2} K_{t, \epsilon, j}\left(\overline{\mathcal{W}_{t, \epsilon}^{-1}}\right)^{-1 / 2}\right\|_{\operatorname{End} L_{2}\left(\mathbb{R}^{n^{*}} / \Gamma^{*} ; L_{2}\left(\mathbb{R}^{n} / \Gamma\right)\right)} \leq C t^{-\epsilon_{2}},
$$

and since the number of $\phi_{t, \epsilon, j}$ is $O\left(t^{n \epsilon_{1}}\right)$, we obtain

$$
\left\|\left(\overline{\mathcal{W}_{t, \epsilon}^{-1}}\right)^{-1 / 2} \sum_{j} K_{t, \epsilon, j}\left(\overline{\mathcal{W}_{t, \epsilon}^{-1}}\right)^{-1 / 2}\right\|_{\text {End } L_{2}\left(\mathbb{R}^{n^{*}} / \Gamma^{*} ; L_{2}\left(\mathbb{R}^{n} / \Gamma\right)\right)} \leq C_{1} t^{n \epsilon_{1}-\epsilon_{2}} .
$$

Choose $\epsilon_{1}$ so that $n \epsilon_{1}-\left(\epsilon \rho / m-2 \epsilon_{1}\right) \leq-3 \epsilon_{1}$. Then (3.28) gives

$$
\left|\left\langle\sum_{j} K_{t, \epsilon, j} u, u\right\rangle_{L_{2}}\right| \leq C_{1} t^{-3 \epsilon_{1}}\left\langle\mathcal{W}_{t, \epsilon}^{-1} u, u\right\rangle_{L_{2}}
$$

and we have

$$
\begin{aligned}
\left\langle\mathcal{W}_{t, \epsilon}^{-1} u, u\right\rangle_{L_{2}} & =\left\langle\mathcal{W}_{t, \epsilon}^{-1}\left(1-t^{-2 \epsilon_{1}}\right) \sum_{j} \phi_{t, \epsilon, j}^{2} u, u\right\rangle_{L_{2}}+t^{-2 \epsilon_{1}}\left\langle\mathcal{W}_{t, \epsilon}^{-1} u, u\right\rangle_{L_{2}} \\
& \geq\left(1-t^{-2 \epsilon_{1}}\right) \sum_{j}\left\langle\mathcal{W}_{t, \epsilon}^{-1} \phi_{t, \epsilon, j} u, \phi_{t, \epsilon, j} u\right\rangle_{L_{2}} .
\end{aligned}
$$

Similarly to (3.27), we deduce from (3.22) and (3.23) that

$$
\begin{aligned}
& \left\langle\mathcal{W}_{t, \epsilon}^{-1} \phi_{t, \epsilon, j} u, \phi_{t, \epsilon, j} u\right\rangle_{L_{2}\left(\mathbb{R}^{n^{*}} / \Gamma^{*} ; L_{2}\left(\mathbb{R}^{n} / \Gamma\right)\right)} \\
& \quad \geq\left(1-C t^{-\epsilon / m_{-}}\right)\left\langle W_{t, \epsilon}^{-1}(-D) u_{j}, u_{j}\right\rangle_{L_{2}\left(U_{t, \epsilon, j}^{+} ; L_{2}\left(\mathbb{R}^{n} / \Gamma\right)\right)},
\end{aligned}
$$

where $u_{j}=\kappa_{j}^{*} \phi_{t, \epsilon, j} u$; therefore (3.29) yields 


$$
\left\langle\mathcal{W}_{t, \epsilon}^{-1} u, u\right\rangle_{L_{2}} \geq\left(1-t^{-\epsilon_{1}}\right) \sum_{j}\left\langle W_{t, \epsilon}^{-1}(-D) u_{j}, u_{j}\right\rangle_{L_{2}}
$$

provided $t$ is large enough.

Fix $\theta_{j}=\theta_{t, \epsilon, j} \in U_{t, \epsilon, j}^{-}$. Due to (3.26) there exists $t_{0}>0$ such that for $t \geq t_{0}$,

$$
\begin{aligned}
& -\left\langle\left((\mathcal{H}-E)^{-1}+C_{0} t^{-\epsilon / m_{-}}\left(\mathcal{H}-E_{0}\right)^{-1}\right) \phi_{t, \epsilon, j} u, \phi_{t, \epsilon, j} u\right\rangle_{L_{2}} \\
\geq & -\left\langle\left(\left(\mathcal{H}\left(\theta_{j}\right)-E\right)^{-1}+t^{-\epsilon_{1}}\left(\mathcal{H}\left(\theta_{j}\right)-E_{0}\right)^{-1}\right) \phi_{t, \epsilon, j} u, \phi_{t, \epsilon, j} u\right\rangle_{L_{2}}
\end{aligned}
$$

Set

$$
\begin{gathered}
\mathcal{B}_{t, \epsilon, j}^{ \pm}=\left(\mathcal{H}\left(\theta_{j}\right)-E\right)^{-1} \pm t^{-\epsilon_{1}}\left(\mathcal{H}\left(\theta_{j}\right)-E_{0}\right)^{-1}, \\
\mathcal{A}_{t, \epsilon, j}^{ \pm}=\left(1 \mp t^{-\epsilon_{1}}\right) W_{t, \epsilon}^{-1}(-D)-t \mathcal{B}_{t, \epsilon, j}^{ \pm} .
\end{gathered}
$$

It follows from (3.30), (3.31) and Lemmas 2.5 and 2.3 that

$$
\mathcal{N}\left(0 ; \mathcal{W}_{t, \epsilon}^{-1}-t\left((\mathcal{H}-E)^{-1}+C_{0} t^{-\epsilon / m_{-}}\left(\mathcal{H}-E_{0}\right)^{-1}\right) ; C^{\infty}\left(\mathbb{R}^{n^{*}} / \Gamma^{*} ; L_{2}\left(\mathbb{R}^{n} / \Gamma\right)\right)\right)
$$

$$
\leq \sum_{j} \mathcal{N}\left(0 ; \mathcal{A}_{t, \epsilon, j}^{+} ; C_{0}^{\infty}\left(U_{t, \epsilon, j}^{+} ; L_{2}\left(\mathbb{R}^{n} / \Gamma\right)\right)\right) .
$$

Since $\phi_{t, \epsilon, j}=1$ on $U_{t, \epsilon, j}^{-}$, for

$$
u=\left(u_{j}\right) \in \bigoplus_{j} C_{0}^{\infty}\left(U_{t, \epsilon, j}^{-} ; L_{2}(\Omega)\right) \subset C^{\infty}\left(\mathbb{R}^{n^{*}} / \Gamma^{*} ; L_{2}(\Omega)\right)
$$

we have

$$
u=\sum_{j} \phi_{t, \epsilon, j} u=\sum_{j} \phi_{t, \epsilon, j} u_{j}
$$

Therefore we can obtain, similarly to (3.32),

$$
\mathcal{N}\left(0 ; \mathcal{W}_{t, \epsilon}^{-1}-t\left((\mathcal{H}-E)^{-1}-C_{0} t^{-\epsilon / m_{-}}\left(\mathcal{H}-E_{0}\right)^{-1}\right) ; C^{\infty}\left(\mathbb{R}^{n^{*}} / \Gamma^{*} ; L_{2}\left(\mathbb{R}^{n} / \Gamma\right)\right)\right)
$$

$$
\geq \sum_{j} \mathcal{N}\left(0 ; \mathcal{A}_{t, \epsilon, j}^{-} ; C_{0}^{\infty}\left(U_{t, \epsilon, j}^{-} ; L_{2}\left(\mathbb{R}^{n} / \Gamma\right)\right)\right)
$$

3.4. Set

$$
\begin{gathered}
B_{t, \epsilon, j, k}^{ \pm}=\left(E_{k}\left(\theta_{j}\right)-E\right)^{-1} \pm t^{-\epsilon_{1}}\left(E_{k}\left(\theta_{j}\right)-E_{0}\right)^{-1}, \\
A_{t, \epsilon, j, k}^{ \pm}=\left(1 \mp t^{-\epsilon_{1}}\right) W_{t, \epsilon}^{-1}(-D)-t B_{t, \epsilon, j, k}^{ \pm} .
\end{gathered}
$$

Since $L_{2}(\Omega)$ can be decomposed into an orthogonal sum of eigenspaces of $\mathcal{H}\left(\theta_{j}\right)$, we deduce from Lemma 2.5 that

$$
\mathcal{N}\left(0 ; \mathcal{A}_{t, \epsilon, j}^{ \pm} ; C_{0}^{\infty}\left(U_{t, \epsilon, j}^{ \pm} ; L_{2}(\Omega)\right)\right)=\sum_{k} \mathcal{N}\left(0 ; \mathcal{A}_{t, \epsilon, j, k}^{ \pm} ; C_{0}^{\infty}\left(U_{t, \epsilon, j}^{ \pm}\right)\right) .
$$

Due to (3.10) the symbol of $A_{t, \epsilon, j, k}^{ \pm}$satisfies

$$
\left|D_{\xi}^{\alpha} a_{t, \epsilon, j, k}^{ \pm}(\xi)\right| \leq C_{\alpha}\left(|\xi|+t^{\epsilon / m_{-}}\right)^{-\rho|\alpha|}\left(W_{t, \epsilon}^{-1}(-\xi)+t\left(E_{k}\left(\theta_{j}\right)-E_{0}\right)^{-1}\right), \quad \forall \alpha,
$$


uniformly in $t \geq 1, k, j$; therefore Theorem 6.1 is applicable. It tells us that for any $\delta \in(0,2 / 3)$ there exist $t_{0}, c, C, C_{1}>0$ such that for all $t \geq t_{0}, k, j$,

$$
\begin{gathered}
\left|\mathcal{N}\left(0 ; \mathcal{A}_{t, \epsilon, j, k}^{ \pm} ; C_{0}^{\infty}\left(U_{t, \epsilon, j}^{ \pm}\right)\right)-F_{t, \epsilon, k}\left(U_{t, \epsilon, j}^{ \pm}\right)\right| \\
\leq C\left(F_{t, \epsilon, k, c, \delta}\left(U_{t, \epsilon, j}^{ \pm}\right)-F_{t, \epsilon, k,-c, \delta}\left(U_{t, \epsilon, j}^{ \pm}\right)\right)+C_{1} F_{t, \epsilon, k, c, \delta}\left(\partial U_{t, \epsilon, j, c, \delta}^{ \pm}\right),
\end{gathered}
$$

where

$$
\begin{gathered}
F_{t, \epsilon, k, c, \delta}(U)=(2 \pi)^{-n} \operatorname{meas}\left\{(\theta, \xi) \in U \times \mathbb{R}^{n} \mid\right. \\
\left.\left(1-t^{-\epsilon_{1}}\right) W_{t, \epsilon}^{-1}(-\xi)-t B_{t, \epsilon, j, k}^{ \pm}<c t^{-\rho \delta \epsilon / m_{-}}\left(W_{t, \epsilon}^{-1}(-\xi)+t\left(E_{k}\left(\theta_{j}\right)-E_{0}\right)^{-1}\right)\right\} \\
F_{t, \epsilon, k}(U)=F_{t, \epsilon, k, 0,0} \\
\partial U_{t, \epsilon, j, c, \delta}^{ \pm}=\left\{x \mid \operatorname{dist}\left(x, \partial U_{t, \epsilon, j}^{ \pm}\right)<c t^{-\rho \delta \epsilon / m_{-}}\right\} .
\end{gathered}
$$

We may choose $\epsilon_{1}$ so that $2 \rho \epsilon / 3 m_{-}>\epsilon_{1}$; then (3.35) yields

$$
\begin{gathered}
\left|\mathcal{N}\left(0 ; \mathcal{A}_{t, \epsilon, j, k}^{ \pm} ; C_{0}^{\infty}\left(U_{t, \epsilon, j}^{ \pm}\right)\right)-F_{t, \epsilon, k, 0}^{1}\left(U_{t, \epsilon, j}\right)\right| \\
\leq C\left(F_{t, \epsilon, k, c}^{1}\left(U_{t, \epsilon, j}\right)-F_{t, \epsilon, k,-c}^{1}\left(U_{t, \epsilon, j}\right)\right)+C_{1} F_{t, \epsilon, k, c}^{1}\left(\partial U_{t, \epsilon, j, c}\right)
\end{gathered}
$$

(with new $c, C, C_{1}>0$ ), where

$$
\begin{gathered}
F_{t, \epsilon, k, c}^{1}(U)=(2 \pi)^{-n} \operatorname{meas}\left\{(\theta, \xi) \in U \times \mathbb{R}^{n} \mid\right. \\
\left.\left(1-t^{-\epsilon_{1}}\right) W_{t, \epsilon}^{-1}(-\xi)-t\left(E\left(\theta_{j}\right)-E\right)^{-1}<c t^{-\epsilon_{1}}\left(W_{t, \epsilon}^{-1}(-\xi)+t\left(E_{k}\left(\theta_{j}\right)-E_{0}\right)^{-1}\right)\right\}, \\
\partial U_{t, \epsilon, j, c}=\left\{x \mid \operatorname{dist}\left(x, \partial U_{t, \epsilon, j}\right)<c t^{-\epsilon_{1}}\right\} .
\end{gathered}
$$

Note that $F_{t, \epsilon, k, c}^{1}(U)=0$ if $k \leq p$ (i.e. if $E_{k}(\theta)-E<0 \quad \forall \theta$ ), and that if $k \geq p+1$ (and hence $E_{k}(\theta)-E>0 \quad \forall \theta$ ), then there exists $c_{1}=c_{1}(c)>0$ such that

$$
F_{k}^{2}\left(t-c_{1} t^{1-\epsilon_{1}} ; U\right) \leq F_{t, \epsilon, k,-c}^{1}(U), \quad F_{k}^{2}\left(t+c_{1} t^{1-\epsilon_{1}} ; U\right) \geq F_{t, \epsilon, k, c}^{1}(U),
$$

where $F_{k}^{2}(t ; U)=(2 \pi)^{-n} \operatorname{meas}\left\{(\theta, \xi) \in U \times \mathbb{R}^{n} \mid W_{t, \epsilon}^{-1}(-\xi)<t\left(E_{k}(\theta)-E\right)^{-1}\right\}$. Further, meas $\partial U_{t, \epsilon, j, c} \leq C_{1} t^{-\epsilon_{1}}$ meas $U_{t, \epsilon, j}$, and therefore

$$
F_{t, \epsilon, k, c}^{1}\left(\partial U_{t, \epsilon, j, c}\right) \leq C_{2} t^{-\epsilon_{1}} F_{k}^{2}\left(t+c_{1} t^{-\epsilon_{1}} ; U_{t, \epsilon, j}\right) .
$$

Now we can rewrite (3.36) as

$$
\begin{gathered}
\mathcal{N}\left(0 ; \mathcal{A}_{t, \epsilon, j, k}^{ \pm} ; C_{0}^{\infty}\left(U_{t, \epsilon, j}^{ \pm}\right)\right) \\
=F_{k}^{2}\left(t ; U_{t, \epsilon, j}\right)\left(1+O\left(t^{-\epsilon_{1}}\right)\right)+O\left(F_{k}^{2}\left(t+c_{1} t^{1-\epsilon_{1}} ; U_{t, \epsilon, j}\right)-F_{k}^{2}\left(t-c_{1} t^{1-\epsilon_{1}} ; U_{t, \epsilon, j}\right)\right),
\end{gathered}
$$

with the $c_{1}$ and the constants in $O$-terms independent of $k, j$ and $t \geq t_{0}$. Note that the RHS in (3.37) is zero if $k \leq p$ and $t$ is large. 
3.5. Set

$$
F_{\epsilon}(t)=\sum_{j, k} F_{k}^{2}\left(t ; U_{t, \epsilon, j}\right)
$$

It follows from (3.8), (3.19), (3.20), (3.32), (3.33) and (3.37) that

$$
\begin{gathered}
N_{+}(t ; H-E ; W)=F_{\epsilon}(t)\left(1+O\left(t^{-\epsilon_{1}}\right)\right) \\
+O\left(F_{\epsilon}\left(t+c t^{1-\epsilon_{1}}\right)-F_{\epsilon}\left(t-c t^{1-\epsilon_{1}}\right)\right)+O\left(t^{n / 2+n \epsilon / m_{-}}\right) .
\end{gathered}
$$

Now, with some $C>0$,

$$
\begin{gathered}
F_{\epsilon}(t)=(2 \pi)^{-n} \sum_{k \geq p+1} \operatorname{meas}\left\{(\theta, \xi) \in \mathbb{R}^{n^{*}} / \Gamma^{*} \times \mathbb{R}^{n} \mid W_{t, \epsilon}^{-1}(-\xi)<t\left(E_{k}(\theta)-E\right)^{-1}\right\} \\
=(2 \pi)^{-n} \sum_{k \geq p+1} \operatorname{meas}\left\{(\theta, \xi) \in \mathbb{R}^{n^{*}} / \Gamma^{*} \times \mathbb{R}^{n} \mid W^{-1}(-\xi)<t\left(E_{k}(\theta)-E\right)^{-1}\right\} \\
+O\left(\sum_{k \geq p+1} \operatorname{meas}\left\{(\theta, \xi) \in \mathbb{R}^{n^{*}} / \Gamma^{*} \times \mathbb{R}^{n}|| \xi \mid \leq C t^{\epsilon / m}, \quad 1<t\left(E_{k}(\theta)-E\right)^{-1}\right\}\right),
\end{gathered}
$$

because if $C$ is sufficiently large then $W^{-1}(-\xi)=W_{t, \epsilon}^{-1}(-\xi)$ for $|\xi| \geq C t^{\epsilon / m}$.

The first term in the RHS of (3.39) is equal to $c_{+}(t ; H-E ; W)$ in $(0.28)$, and the $O$-term admits an upper bound via

$$
C_{1} t^{n \epsilon / m} \operatorname{card}\left\{k \mid k^{2 / n}<C_{2} t\right\} \leq C_{3} t^{n \epsilon / m+n / 2},
$$

since $E_{k}(\theta) \sim$ const $k^{2 / n}$ as $k \rightarrow+\infty$.

Thus, (3.38) and (3.39) give

$$
\begin{gathered}
N_{+}(t ; H-E ; W)=c_{+}(t ; H-E ; W)\left(1+O\left(t^{-\epsilon_{1}}\right)\right) \\
+O\left(c_{+}\left(t+c t^{1-\epsilon_{1}} ; H-E ; W\right)-c_{+}\left(t-c t^{1-\epsilon_{1}} ; H-E ; W\right)\right)+O\left(t^{n / 2+n \epsilon / m}\right) .
\end{gathered}
$$

Under condition (0.19), there exists $c_{0}>0$ such that

$$
c_{+}(t ; H-E ; W) \geq c_{0} t^{n / m},
$$

and since we have chosen $\epsilon>0$ such that $n / 2+n \epsilon / m<n / m$, the last term in (3.40) is included in the $O$-term of (0.29). The second term in the RHS of (3.40) is included in the $O$-term of $(0.29)$ due to $(0.8)$.

Theorem 0.8 is proved.

3.6. Proof of Theorem 0.7. It follows from (0.24) and (0.25) that

$$
c_{+}\left(H-E ; W_{-m}\right) t^{n / m}-c_{+}(t ; H-E ; W)=O\left(t^{-\omega+n / m}\right),
$$

with some $\omega>0$. It follows that $c_{+}(t ; H-E ; W)$ satisfies $(0.8) ;(0.19)$ also follows from (0.24) and (0.25). We see that all the conditions of Theorem 0.8 are satisfied and hence (0.29) holds. Clearly, (0.29) and (3.41) give (0.26).

Theorem 0.7 is proved. 


\section{The CASE OF NON-NEgative $W$}

4.1. Here we prove Theorem 0.9.

This time we have to work with (2.1) because a reduction to both (2.3) and (3.8) is, in general, impossible.

Fix $\epsilon>0$ and $\nu \in(0, \rho)$, take $\mu \in C_{0}^{\infty}(\mathbb{R})$ as in the previous section, and for $c \in[1 / 4,4]$, set

$$
\begin{gathered}
\tilde{\mu}_{t, \epsilon, 0, c}(x)=\mu\left(|x| c^{-1} t^{-\epsilon}\right), \\
\tilde{\mu}_{t, \epsilon,-1, c}(x)=\left(1-\mu\left(|x| c^{-1} t^{-\epsilon}\right)\right) \mu\left(W(x)\langle x\rangle^{m+\nu} c^{-1}\right), \\
\tilde{\mu}_{t, \epsilon, 1, c}(x)=\left(1-\mu\left(|x| c^{-1} t^{-\epsilon}\right)\right)\left(1-\mu\left(W(x)\langle x\rangle^{m+\nu} c^{-1}\right)\right), \\
\mu_{t, \epsilon, j, c}=\tilde{\mu}_{t, \epsilon, j, c}\left(\sum_{j=0, \pm 1} \tilde{\mu}_{t, \epsilon, j, c}^{2}\right)^{-1 / 2}, \\
W_{t, \epsilon, 0}(x)=\tilde{\mu}_{t, \epsilon, 0,2}(x) W(x), \\
W_{t, \epsilon,-1}(x)=\left(1-\mu\left(|x| 4 t^{-\epsilon}\right)\right) \mu\left(W(x)\langle x\rangle^{m+\nu} / 4\right) W(x), \\
W_{t, \epsilon, 1}(x)=\langle x\rangle^{-m-\nu} \mu\left(W(x)\langle x\rangle^{m+\nu} 4\right)+\left(1-\mu\left(W(x)\langle x\rangle^{m+\nu} 4\right)\right) W(x), \\
\left.U_{t, \epsilon, 1}=\left\{x|| x \mid<2 t^{\epsilon}\right\}, \quad U_{t, \epsilon,-1}=\left\{x|| x \mid>t^{\epsilon} / 2, W(x)<2\langle x\rangle^{-m-\nu}\right\}, 2, W(x)>\langle x\rangle^{-m-\nu} / 2\right\} .
\end{gathered}
$$

We have

$$
W_{t, \epsilon, j}(x)=W(x) \quad \forall x \in U_{t, \epsilon, j},
$$

and since the derivatives of $\tilde{\mu}_{t, \epsilon, j, c}, \mu_{t, \epsilon, j, c}$ are supported on a set where $|x|>t^{\epsilon} / 2$, we deduce from $(0.30)$ that

$$
\begin{gathered}
\left|D^{\alpha} \tilde{\mu}_{t, \epsilon, j, c}(x)\right| \leq C_{\alpha}\left(|x|+t^{\epsilon}\right)^{(\nu-\rho)|\alpha|}, \quad \forall \alpha, \\
\left|D^{\alpha} \mu_{t, \epsilon, j, c}(x)\right| \leq C_{\alpha}\left(|x|+t^{\epsilon}\right)^{(\nu-\rho)|\alpha|}, \quad \forall \alpha, \\
\left|D^{\alpha} W_{t, \epsilon, 1}(x)\right| \leq C_{\alpha} W_{t, \epsilon, 1}(x)\left(|x|+t^{\epsilon}\right)^{(\nu-\rho)|\alpha|}, \quad \forall \alpha .
\end{gathered}
$$

4.2. Take $\epsilon_{1} \in(0, \epsilon(\rho-\nu))$, and set

$$
u_{t, \epsilon, j}(x)=\mu_{t, \epsilon, j, 1}(x) u(x), \quad B_{t, \epsilon, \pm C_{0}}=(H-E)^{-1} \pm C_{0} t^{-\epsilon_{1}}\left(H-E_{0}\right)^{-1} .
$$

Using (4.2) and the equality

$$
\sum_{j= \pm 1,0} \mu_{t, \epsilon, j, 1}^{2}=1
$$

we obtain, similarly to (3.12),

$$
\mp\left\langle(H-E)^{-1} u, u\right\rangle_{L_{2}} \geq \mp \sum_{j=0,-1,1}\left\langle B_{t, \epsilon, \pm C_{0}} u_{t, \epsilon, j}, u_{t, \epsilon, j}\right\rangle_{L_{2}},
$$

provided $C_{0}>0$ is large enough. Using (4.4), (4.1) and Lemmas 2.5, 2.3 and 2.4, we deduce from (2.1) the upper bound

$$
\begin{gathered}
N_{ \pm}(t ; H-E ; W) \\
\leq \sum_{j=-1,0,1} \mathcal{N}\left(0 ; I \mp W_{t, \epsilon, j}^{1 / 2} B_{t, \epsilon, \pm C_{0}} W_{t, \epsilon, j}^{1 / 2} ; C_{0}^{\infty}\left(U_{t, \epsilon, j}^{+}\right)\right) \\
\leq \sum_{j=-1,0} \mathcal{N}\left(0 ; I \mp W_{t, \epsilon, j}^{1 / 2} B_{t, \epsilon, \pm C_{0}} W_{t, \epsilon, j}^{1 / 2} ; C_{0}^{\infty}\left(U_{t, \epsilon, j}^{+}\right)\right) \\
\quad+\mathcal{N}\left(0 ; I \mp W_{t, \epsilon, 1}^{1 / 2} B_{t, \epsilon, \pm C_{0}} W_{t, \epsilon, 1}^{1 / 2} ; C_{0}^{\infty}\left(\mathbb{R}^{n}\right)\right) .
\end{gathered}
$$


Similarly, there exists $C_{0}>0$ such that

$$
\begin{gathered}
\mathcal{N}\left(0 ; I \mp W_{t, \epsilon, 1}^{1 / 2} B_{t, \epsilon, \mp C_{0}} W_{t, \epsilon, 1}^{1 / 2} ; C_{0}^{\infty}\left(\mathbb{R}^{n}\right)\right) \\
\leq \sum_{j=-1,0} \mathcal{N}\left(0 ; I \mp W_{t, \epsilon, j}^{1 / 2}(H-E)^{-1} W_{t, \epsilon, j}^{1 / 2} ; C_{0}^{\infty}\left(U_{t, \epsilon, j}^{+}\right)\right) \\
+\mathcal{N}\left(0 ; I \mp t W^{1 / 2}(H-E)^{-1} W^{1 / 2} ; L_{2}\left(\mathbb{R}^{n}\right)\right) \\
=\sum_{j=-1,0} \mathcal{N}\left(0 ; I \mp W_{t, \epsilon, j}^{1 / 2}(H-E)^{-1} W_{t, \epsilon, j}^{1 / 2} ; C_{0}^{\infty}\left(U_{t, \epsilon, j}^{+}\right)\right)+N_{ \pm}(t ; H-E ; W) .
\end{gathered}
$$

4.3. (4.3) and the estimate

$$
\langle x\rangle^{-m-\nu} / 2 \leq W_{t, \epsilon, 1}(x) \leq C\langle x\rangle^{-m}
$$

mean that $W_{t, \epsilon, 1}$ satisfies conditions (0.1) and (0.2) (with $\rho-\nu>0$ instead of $\rho$ ) uniformly in $t \geq 1$. Since $W_{t, \epsilon, 1}$ differs from $W$ only where $W(x) \leq C\langle x\rangle^{-m-\nu}$, we have

$$
c_{-}(t ; H-E ; W)-c_{-}\left(t ; H-E ; W_{t, \epsilon, 1}\right)=O\left(t^{n / m-\omega}\right)
$$

with some $\omega>0$. Further, if $W$ satisfies (0.19), there exists $c_{0}>0$ such that

$$
c_{-}(t ; H-E ; W) \geq c_{0} t^{n / m}
$$

therefore if $c_{-}(t ; H-E ; W)$ satisfies $(0.8)$ then $c_{-}\left(t ; H-E ; W_{t, \epsilon, 1}\right)$ satisfies it as well. Thus, $W_{t, \epsilon, 1}$ satisfies all the conditions of Theorem 0.3 , and the proof of this theorem gives

$$
\mathcal{N}\left(0 ; I+t W_{t, \epsilon, 1}^{1 / 2}(H-E)^{-1} W_{t, \epsilon, 1}^{1 / 2} ; L_{2}\left(\mathbb{R}^{n}\right)\right)=c_{-}\left(t ; H-E ; W_{t, \epsilon, 1}\right)\left(1+O\left(t^{-\omega_{1}}\right)\right),
$$

where $\omega_{1}>0$. Using (4.7), we obtain, with some $\omega_{2}>0$

$$
\mathcal{N}\left(0 ; I+t W_{t, \epsilon, 1}^{1 / 2}(H-E)^{-1} W_{t, \epsilon, 1}^{1 / 2} ; L_{2}\left(\mathbb{R}^{n}\right)\right)=c_{-}(t ; H-E ; W)\left(1+O\left(t^{-\omega_{1}}\right)\right) .
$$

Since

$$
\left(E_{j}(\theta)-E\right)^{-1} \pm C_{0} t^{-\epsilon_{1}}\left(E_{j}(\theta)-E_{0}\right)^{-1}=\left(E_{j}(\theta)-E\right)^{-1}\left(1+O\left(t^{-\epsilon_{1}}\right)\right),
$$

we can similarly obtain, for any $C_{0}>0$,

$$
\mathcal{N}\left(0 ; I+t W_{t, \epsilon, 1}^{1 / 2} B_{t, \epsilon, \pm C_{0}} W_{t, \epsilon, 1}^{1 / 2} ; L_{2}\left(\mathbb{R}^{n}\right)\right)=c_{-}(t ; H-E ; W)\left(1+O\left(t^{-\omega_{1}}\right)\right) .
$$

4.4. By inserting (4.10) into (4.5) and (4.6) we see that (0.9) for $W$ satisfying (0.8), (0.19) and (0.30) will be proved when we show that there exist $C, \omega>0$ such that for $j=0,-1$

$$
\begin{gathered}
\mathcal{N}\left(0 ; I+t W_{t, \epsilon, 1}^{1 / 2} B_{t, \epsilon, \pm C_{0}} W_{t, \epsilon, 1}^{1 / 2} ; C_{0}^{\infty}\left(U_{t, \epsilon, j}\right)\right) \leq C t^{n / m-\omega} \\
\mathcal{N}\left(0 ; I+t W_{t, \epsilon, 1}^{1 / 2}(H-E)^{-1} W_{t, \epsilon, 1}^{1 / 2} ; C_{0}^{\infty}\left(U_{t, \epsilon, j}\right)\right) \leq C t^{n / m-\omega} .
\end{gathered}
$$

Let $P_{1}$ be a spectral projection $P_{(-\infty, E)}(H)$, and set $P_{2}=I-P_{1}$. If $t_{0}$ is sufficiently large and $t \geq t_{0}$, then $P_{2} B_{t, \epsilon, \pm C_{0}} \geq 0$ due to (4.9), and of course $P_{2}(H-E)^{-1} \geq 0$. It follows that for any $N$ there exists $C_{N}>0$ such that

$$
\begin{gathered}
B_{t, \epsilon, \pm C_{0}} \geq P_{1} B_{t, \epsilon, \pm C_{0}} \geq\left(1+C_{1} t^{-\epsilon_{1}}\right) P_{1}(H-E)^{-1} \geq-C_{N}\left(H-E_{0}\right)^{-N}, \\
(H-E)^{-1} \geq-C_{N}\left(H-E_{0}\right)^{-N} .
\end{gathered}
$$


Hence, we may prove (4.11) and (4.12) with $-C_{N}\left(H-E_{0}\right)^{-N}$ instead of $B_{t, \epsilon,-C_{0}}$ and $(H-E)^{-1}$, respectively.

Using Lemma 2.4, the Birman-Schwinger principle and Lemma 2.3, we see that it suffices to prove the estimates

$$
\begin{gathered}
\mathcal{N}\left(0 ;(H-E)^{N}-C_{1, N} t\langle x\rangle^{-m-\nu} ; C_{0}^{\infty}\left(\mathbb{R}^{n}\right)\right) \leq C t^{n / 2-\omega}, \\
\mathcal{N}\left(0 ;(H-E)^{N}-C_{1, N} t \chi_{U_{t, \epsilon, 0}} ; C_{0}^{\infty}\left(\mathbb{R}^{n}\right)\right) \leq C t^{n / 2-\omega},
\end{gathered}
$$

where $\chi_{U_{t, \epsilon, 0}}$ is the characteristic function of $U_{t, \epsilon, 0}$. Upper bounds for the LHS in (4.13) and (4.14) are well-known (see e.g. [19]); one can also apply Lemma 2.3 and the general Theorems 6.1 and 6.2 , and obtain

$$
\begin{aligned}
& \mathcal{N}\left(0 ;(H-E)^{N}-C_{1, N} t\langle x\rangle^{-m-\nu} ; C_{0}^{\infty}\left(\mathbb{R}^{n}\right)\right) \\
\leq C \operatorname{meas}\{(x, \xi) \in & \left.\mathbb{R}^{2 n} \mid(|\xi|+1)^{2 N}<C_{2, N} t\langle x\rangle^{-m-\nu}\right\} \leq C_{3, N} t^{n / 2 N+n /(m+\nu)}, \\
& \mathcal{N}\left(0 ;(H-E)^{N}-C_{1, N} t \chi_{U_{t, \epsilon, 0}} ; C_{0}^{\infty}\left(\mathbb{R}^{n}\right)\right) \\
& \leq C_{3, N} t^{n / 2 N} \operatorname{meas} U_{t, \epsilon, 0} \leq C_{4, N} t^{n / 2 N+n \epsilon} .
\end{aligned}
$$

Since $\nu>0$, we can choose $\epsilon>0$ and $N$ so that (4.13) and (4.14) hold.

4.5. Thus, the first statement of Theorem 0.9, i.e. an analogue of Theorem 0.3 for non-negative $W$, has been proved, and analogues of Theorems 0.7 and 0.8 are proved similarly. The proof of the fact that the $j=1$ terms in (4.5) and (4.6) give the principal terms in the asymptotic formulae remains the same, and to finish the proof, one has to obtain the bounds

$$
\begin{gathered}
\mathcal{N}\left(0 ; I-t W_{t, \epsilon, 1}^{1 / 2} B_{t, \epsilon, \pm C_{0}} W_{t, \epsilon, 1}^{1 / 2} ; C_{0}^{\infty}\left(U_{t, \epsilon, j}\right)\right) \leq C t^{n / m-\omega} \\
\mathcal{N}\left(0 ; I-t W_{t, \epsilon, 1}^{1 / 2}(H-E)^{-1} W_{t, \epsilon, 1}^{1 / 2} ; C_{0}^{\infty}\left(U_{t, \epsilon, j}\right)\right) \leq C t^{n / m-\omega},
\end{gathered}
$$

for $j=-1,0$.

Clearly, there exists $C>0$ such that

$$
-B_{t, \epsilon, \pm C_{0}} \geq-C(-\Delta+1)^{-1}, \quad-(H-E)^{-1} \geq-C(-\Delta+1)^{-1}
$$

therefore we may prove (4.15) and (4.16) with $(-\Delta+1)^{-1}$ instead of $B_{t, \epsilon, \pm C_{0}}$ and $(H-E)^{-1}$, respectively. Now we use Lemma 2.4, the Birman-Schwinger principle and Lemma 2.3, which show that it suffices to obtain the estimates

$$
\begin{gathered}
\mathcal{N}\left(0 ;-\Delta+1-C t\langle x\rangle^{-m-\nu} ; C_{0}^{\infty}\left(\mathbb{R}^{n}\right)\right) \leq C_{1} t^{n / m-\omega}, \\
\mathcal{N}\left(0 ;-\Delta+1-C t \chi_{U_{t, \epsilon, 0}} ; C_{0}^{\infty}\left(\mathbb{R}^{n}\right)\right) \leq C_{1} t^{n / m-\omega} .
\end{gathered}
$$

Upper bounds for the LHS in (4.17) and (4.18) are well-known (see e.g. [19]). One can also apply the general Theorems 6.1 and 6.2 , and obtain

$$
\begin{gathered}
\mathcal{N}\left(0 ;-\Delta+1-C t\langle x\rangle^{-m-\nu} ; C_{0}^{\infty}\left(\mathbb{R}^{n}\right)\right) \\
\leq C \operatorname{meas}\left\{(x, \xi) \in \mathbb{R}^{2 n} \mid(|\xi|+1)^{2}<C_{2} t\langle x\rangle^{-m-\nu}\right\} \leq C_{3} t^{n /(m+\nu)}, \\
\mathcal{N}\left(0 ;-\Delta+1-C t \chi_{U_{t, \epsilon, 0}} ; C_{0}^{\infty}\left(\mathbb{R}^{n}\right)\right) \leq C_{3} t^{n / 2} \operatorname{meas} U_{t, \epsilon, 0} \leq C_{4} t^{n / 2+n \epsilon} .
\end{gathered}
$$

Since $m \in(0,2)$ and $\nu>0$, we can choose $\epsilon>0$ such that (4.17) and (4.18) hold.

Thus, the proof of analogues of Theorems 0.7 and 0.8 for $W \geq 0$ satisfying $(0.30)$ is completed. 
4.6. To obtain an analogue of Theorem 0.6, one has to be more careful because an estimate (4.19) is of no use now: recall that the principal term of the asymptotics is of order $t^{n / 2} \ln t$.

So, we have to repeat all the constructions of this section using $\ln \ln t$ instead of $t^{\epsilon}$. Then in the RHS of (4.19) we have

$$
C_{4} t^{n / 2}(\ln \ln t)^{n \epsilon}=o\left(t^{n / 2} \ln t\right),
$$

and all the errors in other formulae will also be of order $o\left(t^{n / 2} \ln t\right)$.

4.7. Singular perturbations. To prove Theorem 0.10 , it is sufficient to note that due to the condition $(0.31), \mathcal{W}_{1} \in L_{\rho, 0}\left(W \Lambda^{-\epsilon} ; \mathbb{R}^{n^{*}} / \Gamma^{*} ;\right.$ End $\left.L_{2}(\Omega)\right)$; therefore $\mathcal{W}+\mathcal{W}_{1}$ and $\mathcal{W}$ have exactly the same properties as far as the proofs above are concerned.

\section{Some FACTS OF THE THEORY OF PSEUdo DifFERENTIAL OPERATORS}

5.1. Pdo on $\mathbb{R}^{n}$. We need several basic definitions of a general calculus of pdo [10, Section 18] (see also [2]).

Let $\sigma$ be the standard symplectic form on $\mathbb{R}_{X}^{2 n}=\mathbb{R}_{x}^{n} \times \mathbb{R}_{\xi}^{n}$.

A metric $g$ on $\mathbb{R}^{2 n}$ is called $\sigma$-temperate if and only if there exist $C, c>0$ such that

a) $g_{X}(X-Y)<c$ implies $g_{Y} \leq C g_{X}$;

b) $g_{Y}(\cdot) \leq C g_{X}(\cdot)\left(1+g_{Y}^{\sigma}(X-Y)\right)^{C}, \quad \forall X, Y$.

Here

We also require

$$
g_{X}^{\sigma}(Y)=\sup _{Z \neq 0} \frac{\sigma(Y, Z)^{2}}{g_{X}(Z)} .
$$

$$
h(X):=\sup _{Y \neq 0} \frac{g_{X}(Y)}{g_{X}^{\sigma}(Y)} \leq 1, \quad \forall X .
$$

A function $p: \mathbb{R}^{2 n} \rightarrow \mathbb{R}_{+}$is called $\sigma, g$-temperate if and only if there exist $C, c>0$ such that

a) $g_{X}(X-Y) \leq c$ implies $p(X) \leq C p(Y)$

b) $p(X) \leq C p(Y)\left(1+g_{Y}^{\sigma}(X-Y)\right)^{C}, \quad \forall X, Y$.

Below, $H_{j}$ stands for a Hilbert space, and we set $B_{j k}=\operatorname{Hom}\left(H_{j}, H_{k}\right)$.

A function $a \in C^{\infty}\left(\mathbb{R}^{2 n} ; B_{12}\right)$ is said to belong to $S\left(p ; g ; B_{12}\right)$, if and only if for any $k$,

$$
\begin{gathered}
n_{k}(a ; p ; g) \\
:=\sup _{X} \sup _{Y_{j} \neq 0}\left|\left(d^{k} a\right)\left(X ; Y_{1}, \ldots, Y_{k}\right)\right| p(X)^{-1} g_{X}\left(Y_{1}\right)^{-1 / 2} \cdots g_{X}\left(Y_{k}\right)^{-1 / 2}<\infty,
\end{gathered}
$$

where $d^{k} a$ is the $k$-th differential.

$S\left(p ; g ; B_{12}\right)$ is a Fréchet space with $n_{k}(\cdot ; p ; g)$ as seminorms.

If $H_{1}=H_{2}=\mathbb{C}$, we omit $B_{12}$ in the notation and write $S(p ; g)$.

For our purposes, it suffices to use metrics which split:

$$
g_{x, \xi}(y, \eta)=g_{x, \xi}^{\prime}(y)+g_{x, \xi}^{\prime \prime}(\eta),
$$

and some of the results formulated below hold for such metrics only.

Given $a \in S\left(p ; g ; B_{12}\right)$, one can define a pdo $A$ with the (left) symbol $a$ as follows:

$$
(A u)(x)=(2 \pi)^{-n} \int_{\mathbb{R}^{n}} d \xi \int_{\mathbb{R}^{n}} d y \exp \{i(x-y, \xi)\} a(x, \xi) u(y) .
$$


Example 5.1. Let $\rho \in(0,1]$, and let $f$ satisfy (1.2) and (1.3). Then metric

$$
g_{x, \xi}(y, \eta)=|y|^{2}+\langle\xi\rangle^{-2 \rho}|\eta|^{2}
$$

is $\sigma$-temperate, $f$ is a $\sigma, g$-temperate function, and the class $S(f ; g)$ consists of functions satisfying the estimates

$$
\left|D_{x}^{\beta} D_{\xi}^{\alpha} a(x, \xi)\right| \leq C_{\alpha, \beta} f(\xi)\langle\xi\rangle^{-\rho|\alpha|}, \quad \forall \alpha .
$$

The first statement can be easily verified (see e.g. [10, Section 18]), and the second one was, in fact, established when we proved Lemma 1.1. The last statement is evident.

Thus, the class $L_{\rho, 0}\left(f ; \mathbb{R}^{n} ; B\right)$ introduced in Section 2, is a Hörmander class.

In particular, $L_{\rho, 0}\left(\Lambda^{m} ; \mathbb{R}^{n} ; B\right)$ is nothing but Hörmander class $L_{\rho, 0}^{m}\left(\mathbb{R}^{n} ; B\right)$.

Here are the main theorems of the calculus of pdo on $\mathbb{R}^{n}$.

Theorem 5.1. A acts continuously from $\mathcal{S}\left(\mathbb{R}^{n} ; H_{1}\right)$ into $\mathcal{S}\left(\mathbb{R}^{n} ; H_{2}\right)$ and admits a unique continuous extention to an operator from $\mathcal{S}^{\prime}\left(\mathbb{R}^{n} ; H_{1}\right)$ into $\mathcal{S}^{\prime}\left(\mathbb{R}^{n} ; H_{2}\right)$ which is also denoted by $A$.

We write $A \in \Psi\left(p ; g ; B_{12}\right)$.

If $H_{1}=H_{2}=\mathbb{C}$, we omit $B_{12}$ in the notation.

Theorem 5.2. Let $A \in \Psi\left(p ; g ; B_{23}\right)$ and $B \in \Psi\left(q ; g ; B_{12}\right)$ be pdo with the symbols $a, b$ respectively.

Then $A B \in \Psi\left(p q ; g ; B_{13}\right)$, and for any $N$ its symbol $c$ admits the decomposition

$$
c(x, \xi)=\sum_{|\alpha| \leq N-1}(\alpha !)^{-1}\left(\partial_{\xi}^{\alpha} a(x, \xi)\right)\left(D_{x}^{\alpha} b(x, \xi)\right)+r_{N}(x, \xi),
$$

where $r_{N} \in S\left(p q h^{N} ; g ; B_{13}\right)$, and the map

$$
S\left(p ; g ; B_{23}\right) \times S\left(q ; g ; B_{12}\right) \ni(a, b) \mapsto r_{N} \in S\left(p q h^{N} ; g ; B_{13}\right)
$$

is bounded.

Theorem 5.3. Let $A \in \Psi\left(p ; g ; B_{12}\right)$ be a pdo with the symbol a.

Then its formal adjoint $A^{*} \in \Psi\left(p ; g ; B_{21}\right)$, and for any $N$ its symbol admits the decomposition

$$
a^{*}(x, \xi)=\sum_{|\alpha| \leq N-1}(\alpha !)^{-1} i^{|\alpha|} D_{x}^{\alpha} D_{\xi}^{\alpha} a(x, \xi)^{*}+r_{N}(x, \xi),
$$

where $r_{N} \in S\left(p h^{N} ; g ; B_{21}\right)$.

Theorem 5.4. $A \in \Psi\left(1 ; g ; B_{12}\right)$ maps $L_{2}\left(\mathbb{R}^{n} ; H_{1}\right)$ into $L_{2}\left(\mathbb{R}^{n} ; H_{2}\right)$, and there exist $C, N$, which depend only on $n$ and the constants characterizing $p$ and $g$ but not on a, such that

$$
\|A\|_{L_{2}\left(\mathbb{R}^{n} ; H_{1}\right) \rightarrow L_{2}\left(\mathbb{R}^{n} ; H_{2}\right)} \leq C \max _{k \leq N} n_{k}(a ; p ; g) .
$$

Theorem 5.5. Let $m_{0}<m_{1}<\cdots<m_{k}<\cdots$, where $m_{k} \rightarrow+\infty$ as $k \rightarrow+\infty$, and let $a_{j} \in S\left(p h^{m_{j}} ; g ; B_{12}\right)$.

Then there exists $a \in S\left(p h^{m_{0}} ; g ; B_{12}\right)$ such that

$$
a \sim \sum_{j \geq 0} a_{j}
$$


in the sense that for any $N$

$$
a-\sum_{m_{j}<N} a_{j} \in S\left(p h^{N} ; g ; B_{12}\right) .
$$

In Section 2, we also used the following trivial generalization of Theorem 5.5.

Theorem 5.6. Let $m_{j 0}<m_{j 1}<\cdots<m_{j k}<\cdots$, where $m_{j k} \rightarrow+\infty$ as $k \rightarrow+\infty$, and let $m_{00}<m_{10}<\cdots<m_{k 0}<\cdots$, where $m_{k 0} \rightarrow+\infty$ as $k \rightarrow+\infty$.

Let $a \sim \sum_{j \geq 0} a_{j}$ with $a_{j} \in S\left(p h^{m_{j k}} ; g ; B_{12}\right) \quad(j=0,1, \ldots)$ admitting decompositions $a_{j k} \sim \sum_{k \geq 0} a_{j k}$, where $a_{j k} \in S\left(p h^{m_{j k}} ; g ; B_{12}\right)$.

Then $a \sim \sum_{j, k \geq 0} a_{j k}$ in the sense that for any $N$

$$
a-\sum_{m_{j k}<N} a_{j k} \in S\left(p h^{N} ; g ; B_{12}\right) .
$$

In Section 2, we also use the following construction which is justified by Theorem 5.5 .

Let $a \in S_{\rho, 0}\left(f ; \mathbb{R}^{n^{*}} / \Gamma^{*} ; B_{12}\right)$, let

$$
L(s, t)=\sum_{\alpha, \beta} L_{\alpha, \beta} s^{\alpha} t^{\beta} \in \mathbb{C}[[s, t]]=\mathbb{C}\left[\left[s_{1}, \ldots, s_{n} ; t_{1}, \ldots, t_{n}\right]\right]
$$

be a formal power series such that for any $N$, only finitely many $L_{\alpha, \beta}$ with $|\alpha|<N$ are non-zero, and let $P$ be an operator acting continuously in each $S_{\rho, 0}\left(f \Lambda^{k} ; \mathbb{R}^{n} ; B_{12}\right), k \in \mathbb{R}$.

Then Theorem 5.5 allows one to consider the symbol

$$
a_{L}=L\left(D_{\xi}, P\right) a,
$$

which is understood in the sense that

$$
a_{L}-\sum_{|\alpha|<N, \beta} L_{\alpha, \beta} D_{\xi}^{\alpha} P^{\beta} a \in S_{\rho, 0}\left(f \Lambda^{-N \rho} ; \mathbb{R}^{n} ; B_{12}\right) .
$$

Using theorems for the classes $L_{\rho, 0}^{m}\left(\mathbb{R}^{n} ; B\right)$, one deduces theorems for pdo on a compact manifold $M$ without boundary, of the classes $L_{\rho, 0}^{m}(M ; B)$ (see e.g. [10, Section 16]).

In just the same manner one can deduce from theorems for $L_{\rho, 0}\left(f ; \mathbb{R}^{n} ; B\right)$ theorems for the classes $L_{\rho, 0}\left(f ; \mathbb{R}^{n^{*}} / \Gamma^{*} ; B\right)$ introduced in Section 1 .

In the following subsection, we list theorems which are used in the paper.

\subsection{Pdo on a torus.}

Theorem 5.7. $A \in L_{\rho, 0}\left(f ; \mathbb{R}^{n^{*}} / \Gamma^{*} ; B_{12}\right)$ acts continuously from $C^{\infty}\left(\mathbb{R}^{n^{*}} / \Gamma^{*} ; H_{1}\right)$ into $C^{\infty}\left(\mathbb{R}^{n^{*}} / \Gamma^{*} ; H_{2}\right)$, and admits a unique continuous extention to an operator from $\mathcal{D}^{\prime}\left(\mathbb{R}^{n^{*}} / \Gamma^{*} ; H_{1}\right)$ to $\mathcal{D}^{\prime}\left(\mathbb{R}^{n^{*}} / \Gamma^{*} ; H_{2}\right)$.

Theorem 5.8. Let $A \in L_{\rho, 0}\left(f_{1} ; \mathbb{R}^{n^{*}} / \Gamma^{*} ; B_{23}\right)$ and $B \in L_{\rho, 0}\left(f_{2} ; \mathbb{R}^{n^{*}} / \Gamma^{*} ; B_{12}\right)$.

Then $A B \in L_{\rho, 0}\left(f_{1} f_{2} ; \mathbb{R}^{n^{*}} / \Gamma^{*} ; B_{13}\right)$, and its local symbols are expressed via the local symbols of $A, B$ by means of Theorem 5.2.

Theorem 5.9. Let $A \in L_{\rho, 0}\left(f ; \mathbb{R}^{n^{*}} / \Gamma^{*} ; B_{12}\right)$.

Then its formal adjoint $A^{*} \in L_{\rho, 0}\left(f ; \mathbb{R}^{n^{*}} / \Gamma^{*} ; B_{21}\right)$, and its local symbols are expressed via the local symbols of $A$ by means of Theorem 5.3 . 
Theorem 5.10. $A \in L_{\rho, 0}\left(1 ; \mathbb{R}^{n^{*}} / \Gamma^{*} ; B_{12}\right): L_{2}\left(\mathbb{R}^{n^{*}} / \Gamma^{*} ; H_{1}\right) \rightarrow L_{2}\left(\mathbb{R}^{n^{*}} / \Gamma^{*} ; H_{2}\right)$ is bounded.

Theorem 5.11. Let the local symbols of $A \in L_{\rho, 0}\left(f ; \mathbb{R}^{n^{*}} / \Gamma^{*} ; B_{12}\right)$ satisfy the following condition: there exist $C, C_{1}$ such that

$$
\left\|a(\theta, \xi)^{-1}\right\| \leq C f(-\xi), \quad \forall|\xi|>C, \theta,
$$

and let the $A$ be invertible.

Then $A^{-1} \in L_{\rho, 0}\left(f^{-1} ; \mathbb{R}^{n^{*}} / \Gamma^{*} ; B_{12}\right)$.

Theorem 5.12. Let $f$ satisfy (1.2) and (1.3) with $-m_{-}>0$, and let local symbols of $A \in L_{\rho, 0}\left(f ; \mathbb{R}^{n^{*}} / \Gamma^{*} ;\right.$ End $\left.\mathbb{C}^{m}\right)$ satisfy

$$
a(x, \xi)+a(x, \xi)^{*}>c f(-\xi), \quad \forall|\xi|>C, \theta .
$$

Let $A: C^{\infty}\left(\mathbb{R}^{n^{*}} / \Gamma^{*} ; \mathbb{C}^{m}\right) \rightarrow C^{\infty}\left(\mathbb{R}^{n^{*}} / \Gamma^{*} ; \mathbb{C}^{m}\right)$ be symmetric.

Then $A$ is essentially self-adjoint, and its closure is a semibounded (from below) operator with discrete spectrum.

Remark 5.1. Theorem 5.12 is also valid for pdo acting in sections of a finitedimensional fibering over $M$.

Theorem 5.13. Let $A \in L_{\rho, 0}\left(f ; \mathbb{R}^{n^{*}} / \Gamma^{*}\right)$ be a positive definite operator satisfying the conditions of Theorem 5.12, and let $\bar{A}$ be its closure in $L_{2}\left(\mathbb{R}^{n^{*}} / \Gamma^{*}\right)$.

Then $(\bar{A})^{c} \in L_{\rho, 0}\left(f^{c} ; \mathbb{R}^{n^{*}} / \Gamma^{*}\right)$ for any $c \in R$.

Remark 5.2. Theorem 5.13 is also valid for pdo on $\mathbb{R}^{n}$ [2]; in fact, it is proved similarly to the corresponding theorem in [2].

Theorem 5.14. Let $f$ satisfies (1.2) and (1.3).

Then there exists an invertible pdo $A \in L_{\rho, 0}\left(f ; \mathbb{R}^{n^{*}} / \Gamma^{*}\right)$ with

$$
A^{-1} \in L_{\rho, 0}\left(f^{-1} ; \mathbb{R}^{n^{*}} / \Gamma^{*}\right) .
$$

Remark 5.2. Once again this theorem is valid for pdo on $\mathbb{R}^{n}$ as well, and is deduced from its $\mathbb{R}^{n}$-counterpart.

\section{Some General theOREMS ON SPECTRAL ASYMPtotics}

6.1. In Chapter 7 of [16] several general bounds for $\mathcal{N}\left(0 ; A_{t} ; C_{0}^{\infty}\left(\Omega_{t} ; \mathbb{C}^{m}\right)\right)$ were obtained. We shall use only two of them, and not in their full generality.

In both, $A_{t}$ is a symmetric pdo with the symbol $a_{t} \in S\left(p_{t} ; g_{t} ;\right.$ End $\left.\mathbb{C}^{m}\right), g_{t}$ is a $\sigma$-temperate splitting metric, and $p_{t}$ is a $\sigma, g_{t}$-temperate function (all the conditions are satisfied uniformly in $t \geq 1$ ).

Also in both theorems, use was made of the Weyl symbol of $A_{t}$; one can use $a_{t}^{0}:=\left(a_{t}+a_{t}^{*}\right) / 2$ instead (this follows from the more detailed formulation in [16] and a formula expressing the Weyl symbol via the left one).

In the formulations of the theorems, one uses the following functions, depending on constants $c>0, \delta>0$, and a set $U \subset \mathbb{R}^{2 n}$ :

$$
\begin{gathered}
U_{t}\left(g_{t} ; c ; \delta\right)=\left\{(x, \xi) \mid \inf _{(y, \eta) \in U} g_{t ; x, \xi}(x-y, \xi-\eta)<c h_{t}(x, \xi)^{\delta}\right\}, \\
V_{ \pm c, \delta}\left(a_{t}^{0} ; U\right)=(2 \pi)^{-n} \sum_{j=1}^{m} \operatorname{meas}\left\{(x, \xi) \in U \mid \lambda_{j}(x, \xi) \leq \pm c h_{t}(x, \xi)^{\delta} p_{t}(x, \xi)\right\},
\end{gathered}
$$

where the $\lambda_{j}$ are the eigenvalues of $a_{t}^{0}$. 
Theorem 6.1. Let $A_{t}$ be a symmetric pdo with the symbol $a_{t} \in S\left(p_{t} ; g_{t} ;\right.$ End $\left.\mathbb{C}^{m}\right)$, $g_{t}$ a $\sigma$-temperate splitting metric, and $p_{t}$ a $\sigma, g_{t}$-temperate function (all the conditions are satisfied uniformly in $t \geq 1)$. Let

$$
\max _{\mathbb{R}^{2 n}} h_{t}(x, \xi) \rightarrow 0 \quad \text { as } t \rightarrow+\infty,
$$

and let there exist $c>0$ and $R(t)$ such that

$$
a_{t}^{0}(x, \xi):=\left(a_{t}(x, \xi)+a_{t}(x, \xi)^{*}\right) / 2 \geq c p_{t}(x, \xi), \quad \forall|x|+|\xi|>R(t) .
$$

Then for any $\delta \in(0,1 / 3)$, there exist $t_{0}>0, c>0, C$ such that

$$
\begin{gathered}
\left|\mathcal{N}\left(0 ; A_{t} ; C_{0}^{\infty}\left(\Omega ; \mathbb{C}^{m}\right)\right)-V_{0,0}\left(a_{t}^{0} ; \Omega \times \mathbb{R}^{n}\right)\right| \\
\leq C\left(V_{c, \delta}\left(a_{t}^{0} ; \Omega \times \mathbb{R}^{n}\right)-V_{-c, \delta}\left(a_{t}^{0} ; \Omega \times \mathbb{R}^{n}\right)\right)+C V_{c, \delta}\left(a_{t}^{0} ;\left(\partial \Omega \times \mathbb{R}^{n}\right)_{t}\left(g_{t} ; c ; \delta\right)\right) \\
+C \iint h_{t}(x, \xi)^{\delta} d x d \xi,
\end{gathered}
$$

with the integral over $(x, \xi) \in\left(\partial \Omega \times \mathbb{R}^{n}\right)_{t}\left(g_{t} ; c ; \delta\right)$ satisfying

$$
\lambda_{1}(x, \xi) \leq c h_{t}(x, \xi)^{\delta} p_{t}(x, \xi) .
$$

If $m=1$, then the last term in (6.2) may be dropped and one may take any $\delta \in(0,2 / 3)$.

Theorem 6.2. Let $A_{t}$ be a symmetric pdo with the symbol $a_{t} \in S\left(p_{t} ; g_{t} ;\right.$ End $\left.\mathbb{C}^{m}\right)$, $g_{t}$ a $\sigma$-temperate splitting metric, and $p_{t}$ a $\sigma, g_{t}$-temperate function (all the conditions are satisfied uniformly in $t \geq 1)$. Let there exist $C, \epsilon>0$ such that

$$
h_{t}(x, \xi) \leq C(1+|x|+|\xi|)^{-\epsilon}, \quad \forall x, \xi,
$$

and let (6.1) hold.

Then for any $\delta \in(0,1 / 3)$, there exist $t_{0}>0, c>0, C$ such that

$$
\begin{gathered}
\left|\mathcal{N}\left(0 ; A_{t} ; C_{0}^{\infty}\left(\Omega ; \mathbb{C}^{m}\right)\right)-V_{0,0}\left(a_{t}^{0} ; \Omega \times \mathbb{R}^{n}\right)\right| \\
\leq C\left(V_{c, \delta}\left(a_{t}^{0} ; \Omega \times \mathbb{R}^{n}\right)-V_{-c, \delta}\left(a_{t}^{0} ; \Omega \times \mathbb{R}^{n}\right)\right)+C V_{c, \delta}\left(a_{t}^{0} ;\left(\partial \Omega \times \mathbb{R}^{n}\right)_{t}\left(g_{t} ; c ; \delta\right)\right) \\
+C \iint h_{t}(x, \xi)^{\delta} d x d \xi+C,
\end{gathered}
$$

with the integral over $(x, \xi) \in\left(\partial \Omega \times \mathbb{R}^{n}\right)_{t}\left(g_{t} ; c ; \delta\right)$ satisfying

$$
\lambda_{1}(x, \xi) \leq c h_{t}(x, \xi)^{\delta} p_{t}(x, \xi) .
$$

If $m=1$, then the integral in (6.3) may be dropped and one may take any $\delta \in(0,2 / 3)$. 
6.2. The statements of Theorems 6.1 and 6.2 can be reformulated for pdo on a compact manifold without boundary, and the proofs remain essentially the same, being essentially local (even microlocal). The basic ingredient - a construction of an approximate spectral projection $\mathcal{E}$ - is a microlocal procedure, as the proof that the symbol of $\mathcal{E}$ satisfies necessary estimates; these estimates mean that $\mathcal{E}$ belongs to a certain class of pdo. Now it is a class of pdo on $M$; once this fact is established, one has to replace the calculus of pdo on $\mathbb{R}^{n}$ by the one on $M$ and use the same microlocal considerations as in [16].

One can obtain theorems of varying generality; we state one which suffices for our needs.

Let $\rho \in(0,1]$, and let $f: \mathbb{R}^{n} \rightarrow \mathbb{R}_{+}$satisfy the following two conditions.

a) There exists $C>0$ such that

$$
|(\nabla f)(x)| \leq C f(x)\langle x\rangle^{-\rho} .
$$

b) There exist $d_{1}>0, d, m>0, m_{1}$ such that

$$
d_{1}\langle x\rangle^{m_{1}} \leq f(x) \leq d_{1}\langle x\rangle^{m} .
$$

Let $\mathcal{L}$ be a $p$-dimensional Hermitian fibering over a torus $M$, and let $A$ be a symmetric pdo acting in $C^{\infty}(M ; \mathcal{L})$, with local symbols

$$
a_{U, \chi} \in S_{\rho, 0}\left(f ; \mathbb{R}^{n} ; \text { End } \mathbb{C}^{p}\right) .
$$

Let the eigenvalues $\lambda_{j}$ of the local symbols satisfy the estimate

$$
\operatorname{Re} \lambda_{j}(x, \xi) \geq c f(-\xi) \text { for }|\xi|>C,
$$

where $C, c>0$ depend on a chart and a trivialization (one can show that the condition itself is independent of this choice).

Then an analogue of Theorem 5.12 for pdo acting in sections of a finite-dimensional fibering over a torus states that $A$ is essentially self-adjoint, and its closure is a semibounded (from below) operator with discrete spectrum.

Let there also exist functions $\mu_{j}: T^{*} M \rightarrow \mathbb{R}, 1 \leq j \leq p$, such that for any local chart and any trivialization, the eigenvalues of the local symbol satisfy

$$
\left|\lambda_{j}(x, \xi)-\mu_{j}(x, \xi)\right| \leq C f(-\xi)\langle\xi\rangle^{-\omega}
$$

where $C, \omega>0$.

Let $d x d \xi$ be a volume form on $T^{*} M$, and set

$$
\begin{gathered}
V(t)=(2 \pi)^{-n} \sum_{1 \leq j \leq p} \iint_{\mu_{j}(x, \xi)<t} d x d \xi \\
V_{c, \kappa}(t)=\sum_{1 \leq j \leq p} \iint_{\Omega_{j, t, c, \kappa}} d x d \xi \\
J_{c, \kappa}(t)=\sum_{1 \leq j \leq p} \iint_{\Omega_{j, t, c, \kappa}}\langle\xi\rangle^{-\kappa} d x d \xi
\end{gathered}
$$

where

$$
\Omega_{j, t, c, \kappa}=\left\{(x, \xi) \mid \mu_{j}(x, \xi)-t \leq c(t+f(\xi))\langle\xi\rangle^{-\kappa}\right\} .
$$

Note that if we use local charts $\kappa_{j}:\left(\mathbb{R}^{n} \supset\right) U_{j} \rightarrow U_{j}^{1}(\subset M)$ such that $\kappa_{r}^{-1} \circ \kappa_{s}$ are restrictions of orthogonal operators, then all the functions above are well-defined. 
Theorem 6.3. Let (6.4)-(6.8) hold.

Then for any $\delta \in(0,2 / 3)$ there exist $c>0, C$ such that

$$
|N(t ; \bar{A})-V(t)| \leq C\left(V_{c, \kappa}(t)-V_{-c, \kappa}(t)+J_{c, \kappa}(t)\right),
$$

where $\kappa=\min \{\omega, \delta \rho\}$.

6.3. Under additional conditions one can deduce from Theorem 6.3 an asymptotic formula with a remainder estimate.

Theorem 6.4. Let (6.4)-(6.8) hold, and let $V(t)$ satisfy the Tauberian condition (0.8).

Then there exists $\omega>0$ such that

$$
N(t ; \bar{A})=V(t)\left(1+O\left(t^{-\omega}\right)\right) .
$$

Proof. Due to (6.5) and (6.8), there exist $c_{1}>0, C_{1}$ such that

$$
c_{1} t^{n / m_{1}} \leq V(t) \leq C_{1} t^{n / m} .
$$

Therefore it suffices to prove that, with some $C_{2}, \omega>0$.

$$
\begin{gathered}
V_{c, \kappa}(t)-V_{-c, \kappa}(t) \leq C_{2} t^{n / m_{1}-\omega}+C_{2} V(t) t^{-\omega}, \\
J_{c, \kappa}(t) \leq C_{2} t^{n / m_{1}-\omega}+C_{2} V(t) t^{-\omega} .
\end{gathered}
$$

Fix $\epsilon \in\left(0,1 / m_{1}\right)$ and set

$$
\begin{gathered}
\Omega_{j, t, c, \kappa}^{-}=\left\{(x, \xi) \in \Omega_{j, t, c, \kappa}|| \xi \mid<t^{\epsilon}\right\}, \\
\Omega_{j, t, c, \kappa}^{+}=\Omega_{j, t, c, \kappa} \backslash \Omega_{j, t, c, \kappa}^{-}, \\
V_{c, \kappa}^{ \pm}(t)=\sum_{1 \leq j \leq p} \iint_{\Omega_{j, t, c, \kappa}^{ \pm}} d x d \xi, \\
J_{c, \kappa}^{ \pm}(t)=\sum_{1 \leq j \leq p} \iint_{\Omega_{j, t, c, \kappa}^{ \pm}}\langle\xi\rangle^{-\kappa} d x d \xi .
\end{gathered}
$$

Clearly,

$$
\begin{gathered}
V_{c, \kappa}=V_{c, \kappa}^{+}+V_{c, \kappa}^{-}, \quad J_{c, \kappa}=J_{c, \kappa}^{+}+J_{c, \kappa}^{-}, \\
J_{c, \kappa}^{-}(t) \leq C_{3} t^{n \epsilon}, \quad V_{ \pm c, \kappa}^{-}(t) \leq C_{3} t^{n \epsilon}, \\
J_{c, \kappa}^{+} \leq C_{3} V(t) t^{-\kappa \epsilon}, \\
V_{c, \kappa}^{+}(t) \leq V\left(t+c_{2} t^{1-\kappa \epsilon}\right), \quad V_{-c, \kappa}^{+}(t) \geq V\left(t-c_{2} t^{1-\kappa \epsilon}\right)-C_{4} t^{n \epsilon},
\end{gathered}
$$

with some $C_{3}, C_{4}, c_{2}>0$.

Now, (6.11) follows from (6.13), (6.14), (6.16) and (0.8), while (6.12) follows from (6.13).

Theorem 6.4 is proved. 


\section{REFERENCES}

1. Alama, S., Deift, P.A., Hempel, R.: Eigenvalue branches of the Schrödinger operator $H-$ $\lambda W$ in a gap of $\sigma(H)$, Commun. Math. Phys., 121, 291-321 (1989) MR 90c:35046

2. Beals, R.: Weighted distribution spaces and pseudo-differential operators. Journ. Anal. Math., 36, 131-189 (1981) MR 83a:35105

3. Birman, M.S̆.: Discrete spectrum in the gaps of a continuous one for perturbations with large coupling constant In: Estimates and asymptotics for discrete spectra of integral and differential equations. Advances in Soviet mathematics, vol.7, Providence, RI: AMS, 1991, pp. 57-74 MR 95h:47009

4. Birman, M.S.: On the discrete spectrum in the gaps of a perturbed periodic second order operator. Funct. Anal. Appl. 25, No 4, 158-161 (1991)

5. Birman, M.Š., Solomyak, M.Z.: Asymptotics of the spectrum of differential equations. Itogi Nauki: Mat. Anal., vol. 14, VINITI, Moscow, 1977, pp. 5-58; English transl. in J. Soviet Math. 12 (1979), no. 3. MR 57:6892

6. Birman, M.Š., Solomyak, M.Z.: Spectral theory of self-adjoint operators in a Hilbert space. Leningrad: LGU 1980; English transl., Reidel, Dordrecht, 1987. MR 82k:47001; MR 93g:47001

7. Deift, P.A., Hempel, R.: On the existence of eigenvalues of the Schrödinger operator $H-\lambda W$ in a gap of $\sigma(H)$. Commun. Math. Phys., 103, 461-490 (1986) MR 87k:35184

8. Gesztesy, F., Simon, B.: On a theorem of Deift and Hempel. Commun. Math. Phys., 116, 503-505 (1988) MR 89g:35080

9. Hempel, R.: On the asymptotic distribution of eigenvalue branches of the Schrödinger operator $H \pm \lambda W$ in a spectral gap of $H$. J. Reine Angew. Math. 399, 38-59 (1989) MR 90i:35199

10. Hörmander, L.: The analysis of linear partial differential operators. 3. Berlin, New York, Heidelberg: Springer-Verlag 1985 MR 87d:35002a

11. Ivrii, V.: Precise spectral asymptotics for elliptic operators. Lecture Notes in Math. 1100, 1984 MR 86h:58139

12. Khryashchev, S.V.: Asymptotics of the discrete spectrum of the perturbed Hill operator. Zap. LOMI, 147, 188-189 (1985); English transl. in J. Soviet Math. 32 (1986), no. 2. CMP 18:07

13. Khryashchev, S.V.: On the discrete spectrum of the perturbed periodic Schrödinger operator. Zap. LOMI, 190, 157-162 (1991); English transl. in J. Soviet Math. 71 (1994), no. 1. MR 92h:35168

14. Levendorskii, S.Z.: The method of approximate spectral projection. Izv.AN SSSR, Ser.Mat. 49, 1177-1229 (1985); English transl. in Math. USSR Izv. 27 (1986), MR 87c:35127

15. Levendorskï, S.Z.: Non-classical spectral asymptotics. Russian Math. Surveys, 43, No 1, 149-192 (1988) MR 89h:35249

16. Levendorskii, S.Z.: Asymptotic distribution of eigenvalues of differential operators. Dordrecht: Kluwer Academic Publishers. 1990 MR 91m:35176

17. Raikov, G.D.: Eigenvalue asymptotics for the Schrödinger operator with perturbed periodic potential. Inv. Math. 110, 75-93 (1992) MR 93k:35200

18. Reed, M., Simon, B.: Methods of modern mathematical physics. 4. New York, San Francisco, London: Academic Press 1978 MR 58:12429c

19. Rozenbljum, G.V., Solomyak, M.Z., Shubin, M.A. Spectral theory of differential operators. Itogi Nauki i Tekniki: Sovremennye Problemy Mat.: Fundamental'nye Napravleniya, vol. 64, VINITI, Moscow, 1989; English transl., Encyclopaedia of Math. Sci., vol 64 (Partial Differential Equatons, VII, Springer-Verlag, 1994.

20. Seeley, R.T.: Complex powers of an elliptic operator. Singular Integrals, Proc. Symp. Pure Math., vol. 10, Amer. Math. Soc., 1969, pp. 288-307. MR 38:6220

21. Sobolev, A.V.: Weyl asymptotics for the discrete spectrum of the perturbed Hill operator. In: Estimates and asymptotics for discrete spectra of integral and differential equations. Adv. Sov. Math. vol.7, Providence, RI: AMS, 1991, 159-178 MR 95i:34158

Rostov Institute of National Economy, Engels'a 69, 344798, Rostov-on-Don, Russia

E-mail address: leven@ns.rnd.runnet.ru 